Old Dominion University

ODU Digital Commons

Mechanical \& Aerospace Engineering Theses \&

Dissertations

Mechanical \& Aerospace Engineering

Winter 2004

\title{
Computation of Shock Induced Noise in Imperfectly Expanded Supersonic Jets
}

\author{
Bulent Imamoglu \\ Old Dominion University
}

Follow this and additional works at: https://digitalcommons.odu.edu/mae_etds

Part of the Acoustics, Dynamics, and Controls Commons, and the Structures and Materials Commons

\section{Recommended Citation}

Imamoglu, Bulent. "Computation of Shock Induced Noise in Imperfectly Expanded Supersonic Jets" (2004). Doctor of Philosophy (PhD), Dissertation, Mechanical \& Aerospace Engineering, Old Dominion University, DOI: 10.25777/n2jr-7c56

https://digitalcommons.odu.edu/mae_etds/67

This Dissertation is brought to you for free and open access by the Mechanical \& Aerospace Engineering at ODU Digital Commons. It has been accepted for inclusion in Mechanical \& Aerospace Engineering Theses \& Dissertations by an authorized administrator of ODU Digital Commons. For more information, please contact digitalcommons@odu.edu. 


\section{COMPUTATION OF SHOCK INDUCED NOISE IN}

\section{IMPERFECTLY EXPANDED SUPERSONIC JETS}

by

Bulent Imamoglu

B. S. July 1995 , Bogazici University

M. S. July 1998, Bogazici University

A Dissertation Submitted to the Faculty of

Old Dominion University in Partial Fulfillment of the

Requirement for the Degree of

DOCTOR OF PHILOSOPHY

AEROSPACE ENGINEERING

OLD DOMINION UNIVERSITY

December 2004

Approved by:

Oktay Baysal (Director)

$\overline{\text { Ponnampalam Balakumar (member) }}$

Osama A. Kandil (member)

Fang $\mathrm{Hu}$ (member) 


\author{
ABSTRACT \\ COMPUTATION OF SHOCK INDUCED NOISE IN \\ IMPERFECTLY EXPANDED SUPERSONIC JETS \\ Bulent Imamoglu \\ Old Dominion University, 2004 \\ Director: Dr. Oktay Baysal
}

Screech noise exists only in imperfectly expanded jets. The exit pressure of imperfectly expanded jets does not match ambient pressure, so expansion or compression waves appear out of the nozzle and generate shock cell patterns. Screech is generated by the interaction of shock cells and instability waves. Many experiments and computations have been done to model screech noise, but it is not yet a very well known subject.

A numerical study is performed to understand screech generation mechanisms and to compare with latest experiments. A supersonic underexpanded jet of $25.4 \mathrm{~mm}$ diameter is modeled for cases of Mach numbers of 1.19 and 1.43 in axisymmetric two-dimensions. Then the computation is extended to three-dimensions, and Mach numbers of 1.43 again and 1.80 are solved. Full Navier-Stokes equations are solved in cylindrical coordinates, and large eddy simulation (LES) turbulence modeling is added for axisymmetric cases. For spatial discretizations, fifth order Weighted Essentially Non Oscillatory (WENO) scheme is used because it is a suitable method for capturing shocks. Time discretization is third order time total variation diminishing (TVD) scheme, which is accurate enough, and needs considerably lower storage than fourth order schemes. These methods do not require any artificial viscosity or tune up parameters.

The experimental results have predicted that the solution is in axisymmetric mode for Mach 1.19 and in helical (three-dimensional) mode for 1.43. However, our solution for Mach 1.43 has produced satisfactory results. Frequency analysis has been done by taking fast Fourier transforms of pressure history data. The experimental screech frequencies of $8400 \mathrm{~Hz}$ for Mach 1.19 and $5400 \mathrm{~Hz}$ for Mach 1.43 have been verified 
with computational results. Computed shock cell structure is in agreement with experiments and other computations in all cases. The screech waves emerge from the second and third shock cells, like in the experiments. The screech wavelength can be roughly estimated as 1.5 D, which is close to other computational studies. In Mach 1.80 case, barrel shock concept is observed as in the experiments. Three dimensional effects are investigated by creating an animation of planes of varying azimuth angles.

This study has been a good verification of existing experimental results. It has also made a contribution, since it is a method to calculate screech frequencies without artificial damping or other tune-up parameters. Also, the visual data obtained by the animations has been useful to see shock generation locations. 


\section{ACKNOWLEDGEMENTS}

I would like to thank Dr. Oktay Baysal for directing my dissertation and for his help and support during my $\mathrm{PhD}$ program at Old Dominion University.

I would like to thank Dr. Ponnampalam Balakumar for his guidance and support on this study and his time to help and answer my questions.

I would like to thank Dr. Osama A. Kandil and Dr. Fang Hu for serving in my dissertation committee, reviewing my dissertation and for their valuable recommendations.

Thanks also to my friends, Ilker Turkgeldi, Ilhan \& Tuba Bayraktar, and Ilteris Koc for making my stay in Norfolk enjoyable.

Finally, I would like to thank my family for their great support during my study at Old Dominion University. 


\section{NOMENCLATURE}

a

A

c

D

E

F, G, H

H, W

k

M

$\mathrm{p}$

p

R

$\mathrm{S}$

$\mathrm{t}$

$\mathrm{T}$

$\mathrm{u}, \mathrm{v}, \mathrm{w}$

$\mathrm{U}$

v

$\mathrm{x}$

y

z

\section{Greek Letters}

$\beta$

$\zeta, \eta$

$\theta$

$\mu$
Speed of sound

Area

ENO coefficients

Diameter

Total energy

Flux vectors in $\mathrm{x}, \mathrm{y}, \mathrm{z}$ directions

Height and width of computation domain

Conduction coefficient

Mach number

Pressure

Grid generation parameter

Gas constant

Stencil

Time

Temperature

Velocity in $\mathrm{x}, \mathrm{y}, \mathrm{z}$ directions

Flux vector in time

A general function of coordinates (Chapter 4.1, 4.2)

Cartesian coordinate or cylindrical axial

Cartesian coordinate or cylindrical radial

Cartesian coordinate or cylindrical azimuthal
Smooth indicator

Transformed coordinates

Azimuth angle

Viscosity 

$\rho$
Density
$\tau$
Stress tensor
$\omega$
Weight coefficient

Superscripts and subscripts

$\begin{array}{ll}\text { super * } & \text { Throat conditions } \\ \text { sub e } & \text { Exit conditions } \\ \text { sub V } & \text { Viscous flux }\end{array}$




\section{TABLE OF CONTENTS}

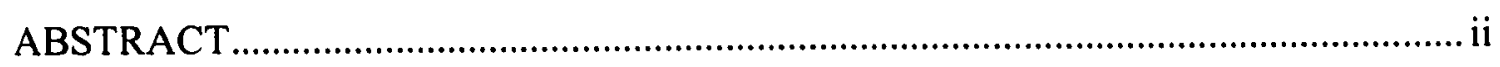

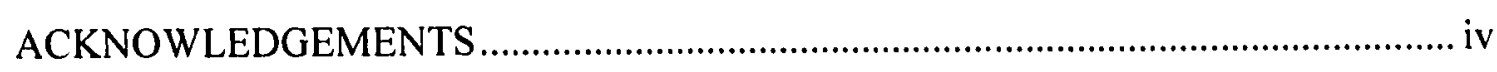

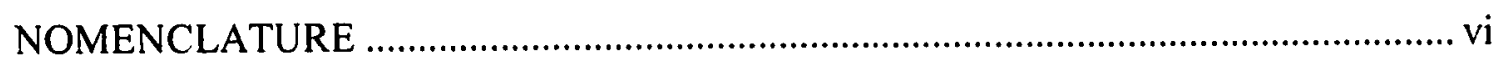

TABLE OF CONTENTS..........................................................................................

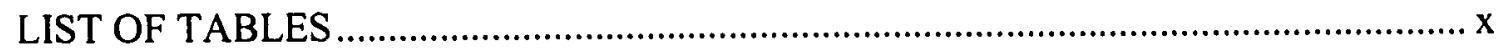

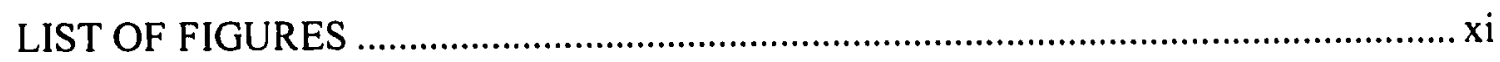

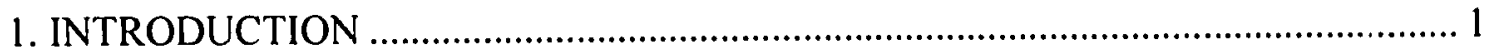

1.1 Characteristics of Supersonic Jet Noise ................................................................. 1

1.2 Imperfectly Expanded Jets............................................................................ 2

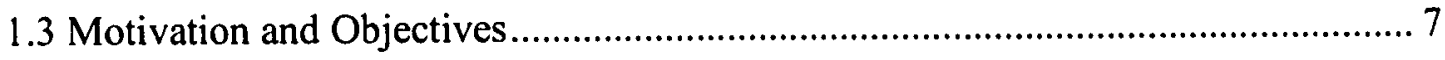

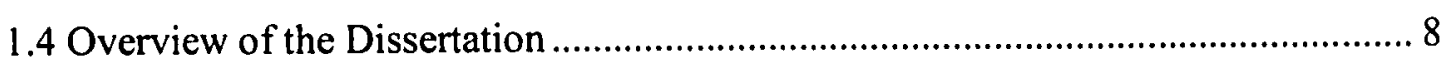

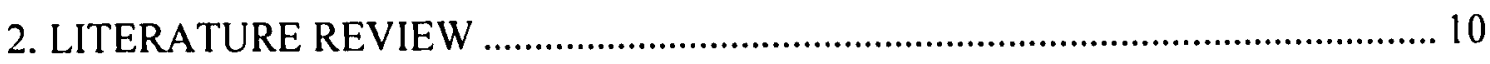

2.1 Experimental Studies on Jet Screech ............................................................... 10

2.2 Numerical Studies on Jet Screech........................................................................ 12

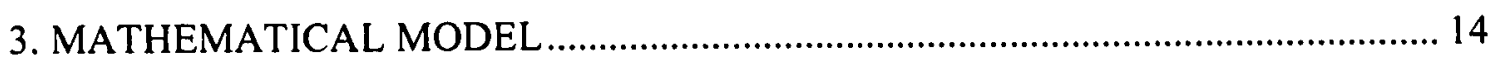

3.1. Axisymmetric model .................................................................................... 14

3.1.1 Initial and boundary conditions ............................................................ 15

3.1.2 Turbulence modeling .............................................................................. 16

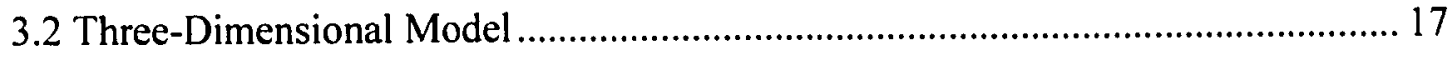

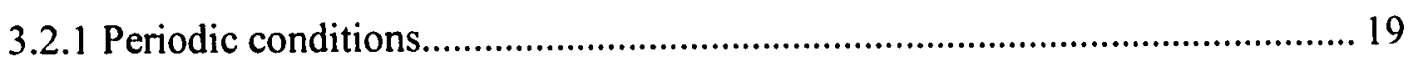

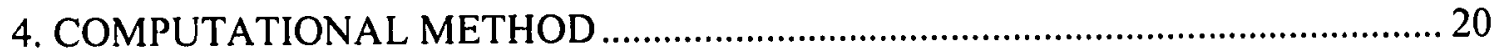

4.1. Development of ENO and WENO schemes ....................................................... 20

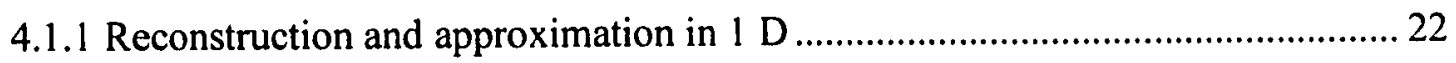

4.1.2 One Dimensional Reconstruction .................................................................... 23

4.1.3 One Dimensional Conservative approximation ................................................. 27

4.2 Spatial Discretization ..................................................................................... 28

4.3 Temporal Discretization .............................................................................. 31

viii 


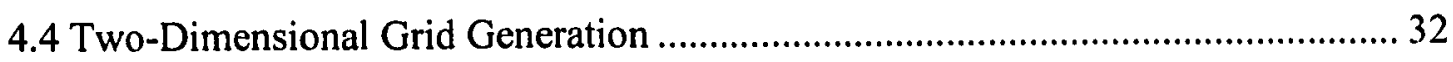

4.5 Three-Dimensional Grid Generation ........................................................................ 37

5. TWO-DIMENSIONAL SIMULATION RESULTS ...................................................... 39

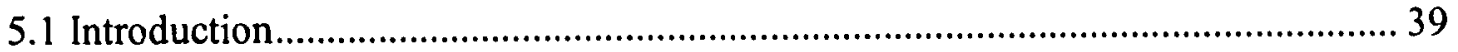

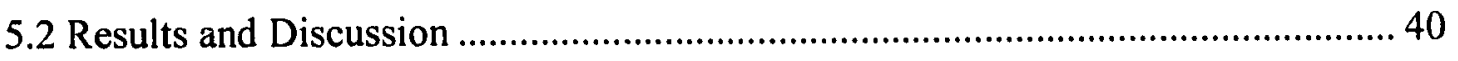

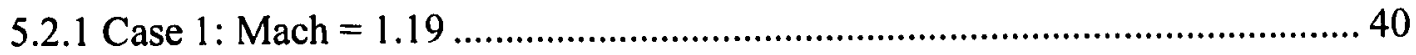

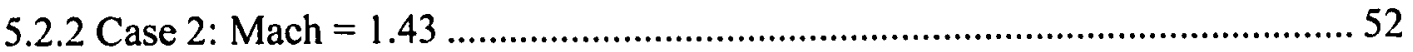

6. THREE-DIMENSIONAL SIMULATION RESULTS ……………...........................5 57

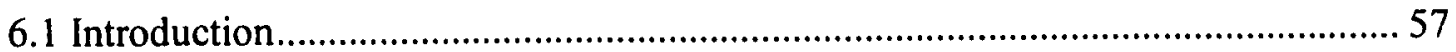

6.2 Results and Discussion ...................................................................................... 57

7. CONCLUSIONS AND RECOMMENDATIONS …...................................................... 69

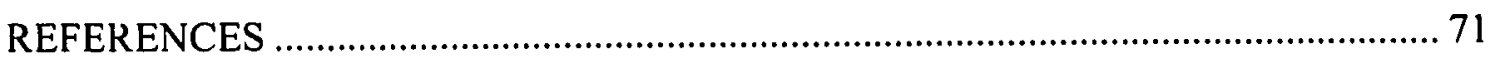

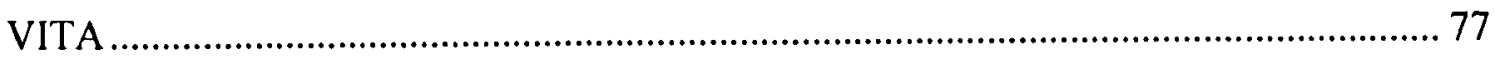




\section{LIST OF TABLES}

Table 1: ENO Coefficients..

26 


\section{LIST OF FIGURES}

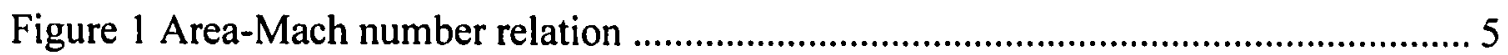

Figure 2: Ghost points for periodic boundary condition................................................ 19

Figure 3: Fifth order WENO discretization .................................................................. 31

Figure 4: Computational domain and boundary conditions..........................................33

Figure 5 Grid generation function for $\mathrm{x}$-direction ..................................................... 34

Figure 6 Grid generation function in the $y$-direction................................................. 36

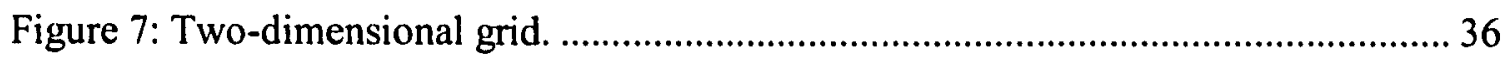

Figure 8: Computational domain and grid for 3-D simulation. ......................................... 38

Figure 9: Time averaged density contours after 40000 steps. ........................................... 41

Figure 10: Time averaged pressure contours after 40000 steps......................................... 41

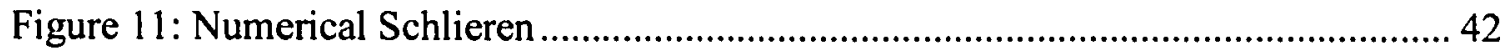

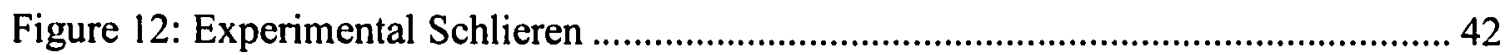

Figure 13: Instantaneous pressure contours, screech waves.......................................... 43

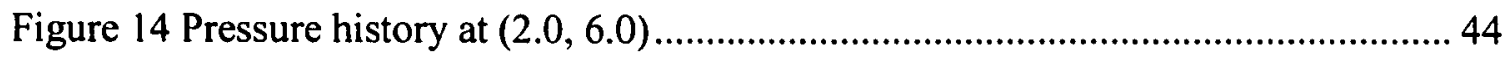

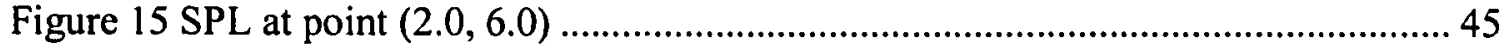

Figure 16a: Initial frame of animation, density contours............................................... 46

Figure 17 Instantaneous density contours after 150,000 time steps. ............................. 52

Figure 18 Instantaneous pressure contours after 150,000 time steps................................ 53

Figure 19 Time averaged density contours after 150,000 time steps. ….......................... 54

Figure 20 Time averaged pressure contours after 150,000 steps..................................... 54

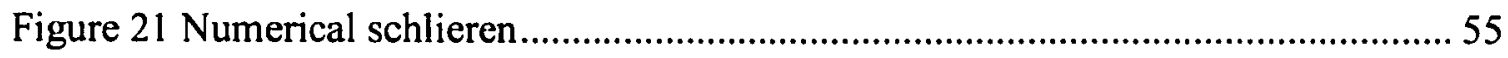

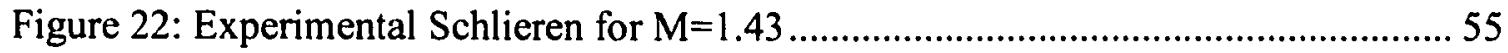

Figure 23 Comparison with experimental results. ........................................................ 56

Figure 24: Time averaged density contours after 30000 steps, Mach $=1.80$..................... 58

Figure 25: Instantaneous density contours after 32000 steps, Mach $=1.80 \ldots \ldots \ldots \ldots \ldots \ldots . . . . .59$

Figure 26: Time averaged density contours after 150000 steps, Mach $=1.43 \ldots \ldots \ldots \ldots \ldots . . . . .60$

Figure 27: Instantaneous density contours 150000 steps, Mach $=1.43 \ldots \ldots \ldots \ldots \ldots \ldots \ldots \ldots . . . . .60$

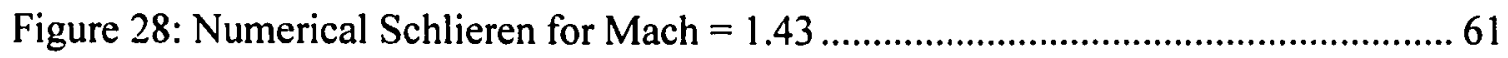




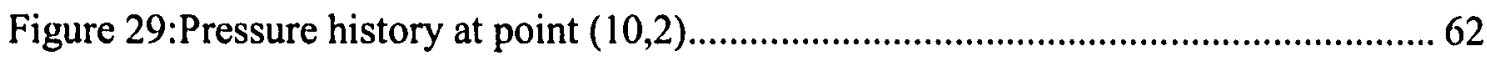

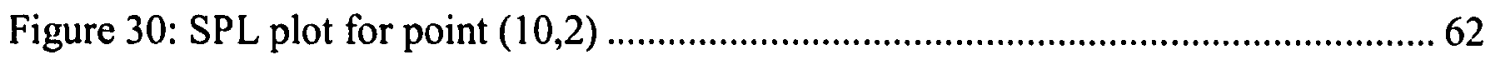

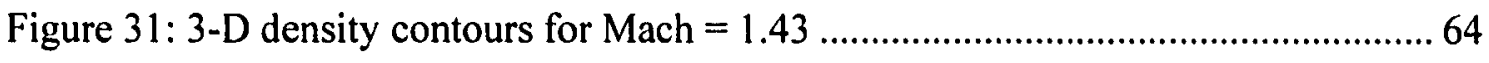

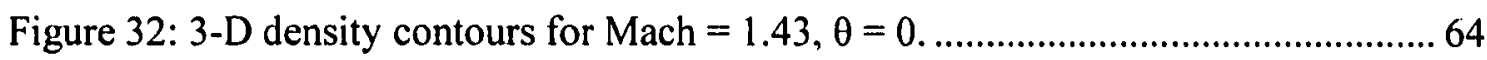

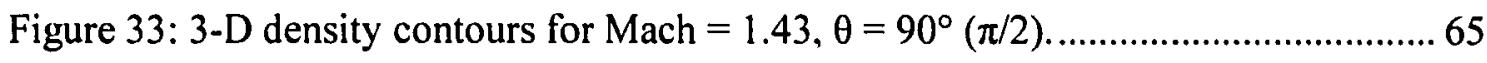

Figure 34: Comparison of Fig. 8 and Fig. 9, number of contours are reduced for clarity. 65 Figure 35: Variation of density in azimuthal direction at selected point $(2.12,0.86)$...... 66 Figure 36: Variation of density in azimuthal direction at selected point $(4.00,0.26)$..... 67 Figure 37: Comparison of 3-D and 2-D computations with experiment for Mach $=1.43$ at

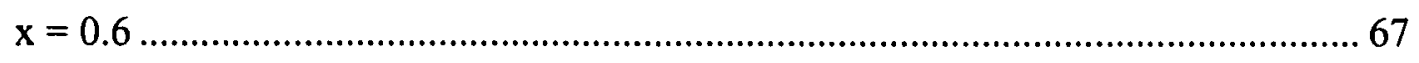

Figure 38: Comparison of 3-D and 2-D computations with experiment for Mach $=1.43$ at

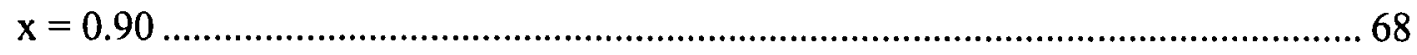




\section{CHAPTER 1 INTRODUCTION}

An imperfectly expanded supersonic jet contains instability vortices and shock cell patterns. As these shocks and vortices react with perturbations, screech noise is generated. These screech tones will produce further perturbations and, therefore, form a feedback loop. This process occurs in 4 steps:
1) Amplification of instability waves
2) Interaction of disturbances with shock cell structure
3) Back propagation of acoustic disturbance to nozzle lip
4) Conversion of acoustic disturbance into instability waves and loop closes.
Noise is generated in step 2.

To better understand screech concept, the characteristics of supersonic jet noise will be introduced briefly, and then, imperfectly expanded (overexpanded or underexpanded) jets will be investigated.

\subsection{Characteristics of Supersonic Jet Noise}

Unless a supersonic jet issuing from a convergent-divergent nozzle is operated very close to the nozzle design condition, its noise spectrum includes discrete and broadband components. The discrete components are called screech tones. When strong shock cells are present, the screech tone is accompanied by its harmonics. The screech component disappears when the jet is perfectly expanded. The noise spectrum of a perfectly expanded jet is made up of broad, smooth peak. This broadband noise component is generated by the turbulence in the mixing layer, and therefore called the turbulent mixing noise. If the jet operating conditions are mocified to be off-design, additional broadband noise will be emitted, which is originated by a quasi-periodic shock-cell structure inside the jet plume. This noise component is called broadband 
shock-associated noise. When screech noise is present, it dominates all other jet noise components. Our study is focused on imperfectly expanded jets, and screech noise.

\subsection{Imperfectly Expanded Jets}

To introduce the concept of imperfectly expanded jets, we consider the quasione-dimensional, isentropic flow in a converging-diverging nozzle. Quasi-onedimensional flow means that all flow variables and the area are functions of $x$ only:

$$
A=A(x), \quad p=p(x), \quad \rho=\rho(x), \quad u=u(x)
$$

For a steady flow, between two sections of the duct 1 and 2 , the conservation of mass requires that

$$
\rho_{1} u_{1} A_{1}=\rho_{2} u_{2} A_{2}
$$

In differential form, the continuity equation becomes

$$
\mathrm{d}(\rho \mathrm{A})=0
$$

or

$$
\frac{d \rho}{\rho}+\frac{d u}{u}+\frac{d A}{A}=0
$$

The momentum equation between a stage with $p, A, u, \rho$ and another stage with $p+d p, A+d A, u+d u, \rho+d \rho$ over an incremental distance $\Delta x$ can be written as:

$$
p A+\rho u^{2} A+p d A=(p+d p)(A+d A)+(\rho+d \rho)(u+d u)^{2}(A+d A)
$$


Dropping all second order terms, and combining with the continuity equation, the momentum equation simplifies to

$$
d p=-\rho u d u
$$

This form is known as Euler's Equation.

Introducing the speed of sound, $a$, and Mach number, $M=u / a$ (ratio of speed to speed of sound), further arrangements can be made (Anderson, 1990) to obtain the areavelocity relation:

$$
\frac{d A}{A}=\left(M^{2}-1\right) \frac{d u}{u}
$$

This is an important result that tells a lot about the physics of compressible flow. Depending on the value of Mach number M, flow conditions change significantly.

1. When $M \rightarrow 0$, the area velocity relation simplifies to $A u=$ const., which corresponds to incompressible flow.

2. When $0 \leq \mathrm{M}<1$, the flow is subsonic, and an increase in velocity is associated with a decrease in area. As in incompressible flow, the velocity increases in a converging duct and decreases in a diverging duct.

3. When $M>1$, the flow is supersonic, and an increase in velocity is associated with an increase in area, and vice versa. The flow behaves just opposite way to incompressible flow. The velocity increases in a diverging duct and decreases in a converging duct.

4. When $M=1$, the flow is sonic and the area-velocity relation becomes $\mathrm{dA} / \mathrm{A}=0$. This corresponds to a minimum or a maximum in the area distribution, however, the physically realistic solution is that the sonic condition occurs at a minimum of the area distribution. 
These results show that a gas can expand isentropically from subsonic to supersonic speeds only by flowing through a converging-diverging duct; and the flow at the minimum area section of the duct must be sonic. This minimum area is called throat. Similarly, a gas can compress isentropically from supersonic to subsonic speed by flowing through a converging-diverging duct with a sonic throat. A converging-diverging nozzle is called De Laval nozzle, after Carl G. P. de Laval, who used this configuration in steam turbines in the late $19^{\text {th }}$ century. The convergingdiverging geometry is also used in rocket engines, supersonic wind tunnels and other supersonic applications.

If we denote the throat conditions of a converging-diverging duct by asterisk, then the throat area is $A^{*}, M^{*}=1$, and $u^{*}=a^{*}$. Between the throat and any other section of the duct, the conservation of mass can be written as

$$
\rho^{*} u^{*} A^{*}=\rho u A
$$

Using that $\mathrm{u}^{*}=\mathrm{a}^{*}$, we can write

$$
\frac{A}{A^{*}}=\frac{\rho^{*}}{\rho_{0}} \frac{\rho_{0}}{\rho} \frac{a^{*}}{u}
$$

where $\rho_{0}$ is the stagnation density. Using isentropic relations and rearranging, (Anderson, 1990), the area-Mach number relation is obtained:

$$
\left(\frac{\mathrm{A}}{\mathrm{A}^{*}}\right)^{2}=\frac{1}{\mathrm{M}^{2}}\left[\frac{2}{\gamma+1}\left(1+\frac{\gamma-1}{2} \mathrm{M}^{2}\right)\right]^{(\gamma+1) /(\gamma-1)}
$$

This relation tells that the Mach number at any location in the duct is a function of the ratio of local duct area to sonic throat area. Also, $A \geq A^{*}$; and there are two Mach 
number values for any area ratio greater than 1, one is subsonic and the other is supersonic. Equation (1.10) is graphed in Figure 1.

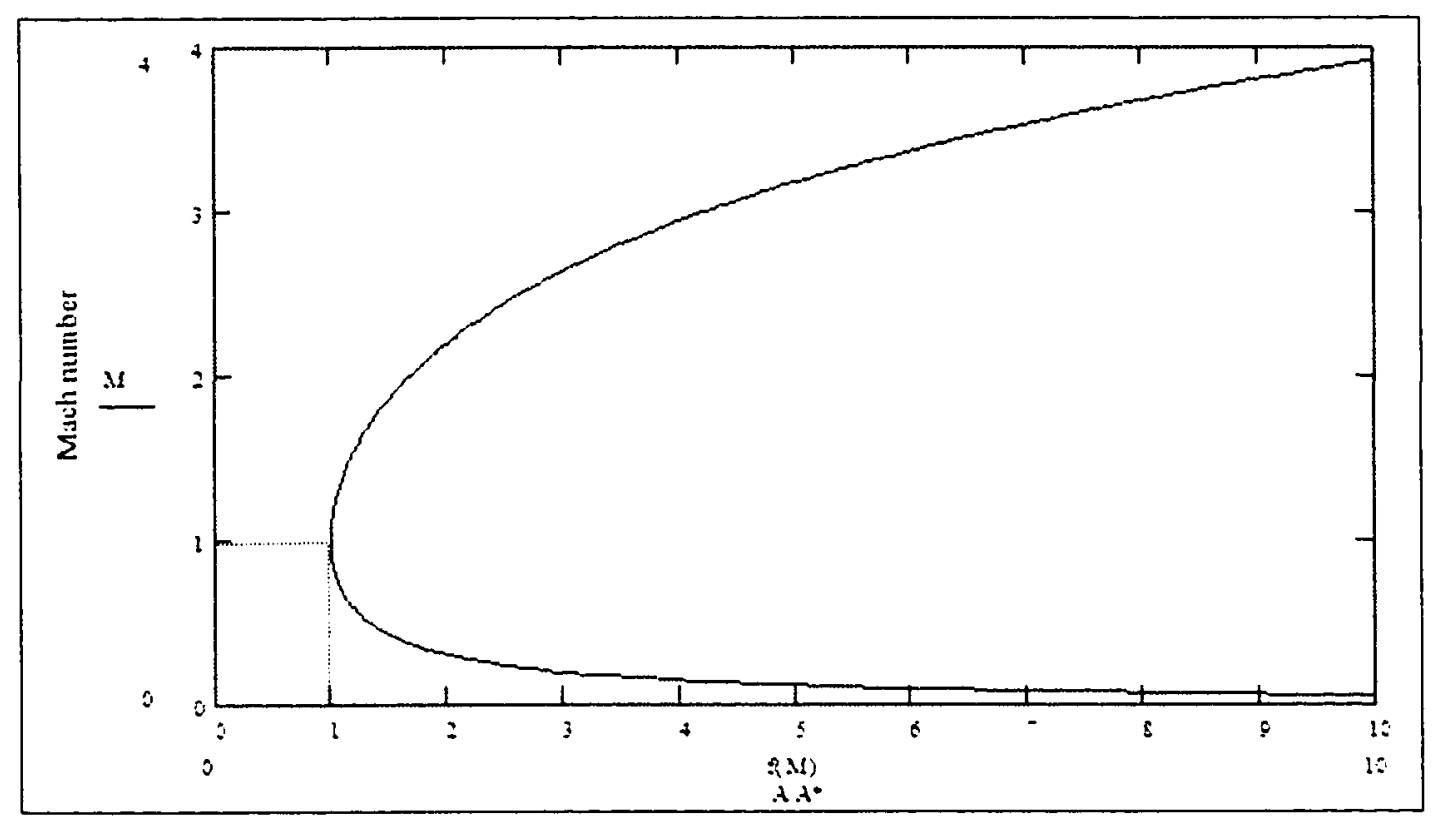

Figure 1 Area-Mach number relation

Let us consider a converging-diverging nozzle with fixed inlet pressure $\mathrm{p}_{0}$ and adjustable exit pressure $p_{c}$. If these two are equal, there will be no flow through the nozzle. When $p_{e}$ is decreased slightly under $p_{0}$, air will start to flow due to the pressure difference. The flow is subsonic everywhere and the maximum Mach number is observed at the throat. The actual area of the throat, $A_{t}$, is larger than the sonic throat area, $A^{*}$. When the exit pressure is reduced further, the Mach number everywhere in the nozzle and the mass flow through the nozzle will keep increasing until the throat becomes sonic. Let us call this exit pressure $\mathrm{p}_{\mathrm{e} 1}$. At this point, the flow is still subsonic everywhere except the throat. The mass flow rate at the throat is given by

$$
\dot{\mathrm{m}}=\rho_{t} A_{t} u_{t}=\rho^{*} A^{*} a^{*}
$$

The Mach number cannot increase above 1 at the throat when $p_{\mathrm{c}}$ is further reduced below $\mathrm{p}_{\mathrm{el}}$, due to area velocity relation (1.7). Therefore, the flow properties upstream 
of the throat are frozen for any exit pressure below $\mathrm{p}_{\mathrm{el}}$, the flow is called choked flow. The mass flow remains constant with decreasing exit pressure after the flow becomes choked.

When the exit pressure is decreased below $\mathrm{p}_{\mathrm{el}}$, nothing happens upstream of the throat, but a lot happens downstream. When the exit pressure is below $\mathrm{p}_{\mathrm{el}}$ but still too high to allow an isentropic supersonic flow in the divergent nozzle, a normal shock exists in the divergent part. Shock location varies with the back pressure, such that the subsonic flow behind the shock achieves the exit pressure. As the exit pressure is reduced further, the shock will move downstream. The shock will be at the exit when the exit pressure reaches $p_{\mathrm{e} 2}$, the static pressure behind a normal shock at the design Mach number of the nozzle. Let $\mathrm{p}_{\mathrm{e} 3}$ be the proper isentropic value for the design exit Mach number. When the exit pressure is further reduced, but still above $p_{\mathrm{e} 3},\left(p_{\mathrm{e} 2}<\mathrm{p}<\mathrm{p}_{\mathrm{e} 3}\right)$, the flow inside the nozzle is supersonic, but the pressure increase from $p_{\mathrm{e} 2}$ to $\mathrm{p}_{\mathrm{e}}$ takes place across an oblique shock attached to the nozzle exit, outside the nozzle. This is called an overexpanded nozzle flow. When the exit pressure is reduced to the isentropic value $p_{\mathrm{e} 3}$, no waves will exist at the exit, the flow will be purely isentropic. When the exit pressure is reduced below $p_{\mathrm{c} 3}$, the nozzle is said to be underexpanded, because the pressure just before the exit is higher than the back pressure, so the flow continues to expand after the nozzle. To make a summary, as the back pressure is decreased, the following is observed:

1. Subsonic flow $\left(\mathrm{p}_{\mathrm{e}}>\mathrm{p}_{\mathrm{el}}\right)$

2. Choked flow $\left(p_{\mathrm{e}}=p_{\mathrm{el}}\right)$

3. Normal shock in divergent part $\left(\mathrm{p}_{\mathrm{e} 1}<\mathrm{p}_{\mathrm{e}}<\mathrm{p}_{\mathrm{e} 2}\right)$

4. Normal shock at exit $\left(p_{e}=p_{e 2}\right)$

5. Oblique shock at exit, overexpanded $\left(\mathrm{p}_{\mathrm{e} 2}<\mathrm{p}_{\mathrm{e}}<\mathrm{p}_{\mathrm{e} 3}\right)$

6. Isentropic flow $\left(p_{e}=p_{e 3}\right)$

7. Expansion waves at exit, underexpanded $\left(p_{e}<p_{e 3}\right)$

The expansion waves of the underexpanded jets are followed by compression waves and expansion waves again, etc. Similarly, the oblique shock at exit of the overexpanded jet is a compression wave and it is followed by expansion waves, and 
compression, expansion, etc. These successive expansion and compression waves make up the shock cell structures that generate the screech noise; and they will be observed in our computations in following chapters.

\subsection{Motivation and Objectives}

There have been many experimental and numerical studies about screech noise in imperfectly expanded jets. However, sound generation is still not well understood. Recent experiments by Panda (1999) have revealed even more detailed information about screech and new observations. Some of these are:

* There is interference between upstream propagating sound wave and downstream propagating hydrodynamic waves.

* Standing wavelength is not equal to shock spacing.

* Fluctuations and convective velocity of organized vortices match standing wavelength rather than shock spacing.

Some of the unresolved questions after those observations are:

* What is the exact nature of the feedback loop?

* Where do the effective sound sources exist?

* How do the shocks respond to a periodic train of disturbances?

* How do the sound waves generate more periodic disturbances in the flow?

Our objective to solve the screech problem numerically for the same cases as in (Panda, 1999), to make comparisons with these experiments as well as other numerical results; and try to understand the mechanisms that generate shock induced noise in imperfectly expanded jets. 


\subsection{Overview of the Dissertation}

A brief introduction to jet noise, imperfectly expanded jets, objective of the dissertation have been given in this chapter. Chapter 2 is a literature survey of experimental and numerical studies about screech noise.

In Chapter 3, the mathematical model, governing equations, initial and boundary conditions, turbulence modeling, and extension of the problem to three dimensions are presented. Full Navier-Stokes equations in cylindrical coordinates, axisymmetric and three-dimensional, and Large Eddy Simulation (LES) sub grid scale model has been used. Initial and boundary conditions match the experimental conditions.

Chapter 4 is about computational methods. Essentially Non Oscillatory (ENO) and Weighted ENO (WENO) schemes are introduced. Spatial discretization used in this study, fifth order WENO scheme, and temporal discretization by third order TVD Runge-Kutta scheme are presented. Two- and three-dimensional grids and the algebraic methods to generate them are explained.

In chapter 5, results of two-dimensional (axisymmetric) computations are shown. These include underexpanded jets of Mach numbers 1.19 and 1.43. Comparison with experiments and other numerical studies are made. Even though 1.43 Mach number is a three-dimensional case, axisymmetric computations give reasonable results. Contour plots of density and pressure are generated for given instants and for time-average. Time-frame animations are generated by adding the instantaneous contours one after another. Numerical Schlieren graph is generated by plotting density gradient, to make comparisons with Schlieren photographs. Screech frequency has been computed by taking fast Fourier transform of pressure history at selected points. 
In chapter 6, three-dimensional results are presented. The first case is Mach 1.80 , which is solved to demonstrate the barrel shock occurrence and to verify the capability of the code to capture shocks at higher speeds. The second one is Mach 1.43 , solved again in three dimensions. The three-dimensional effects are investigated by creating an animation of contour plots in the angular direction. Similar graphs are generated and screech frequency is computed as in the previous chapter.

Chapter 7 is the last one in this dissertation. It contains conclusions of this study and recommendations for future work. 


\section{CHAPTER 2 \\ LITERATURE REVIEW}

There have been many experimental studies on shock induced noise since screech concept was discovered in 1953. Numerical studies began as computers became powerful in the late 1980's. In this chapter, a summary of significant experimental and numerical studies on shock induced noise will be presented.

\subsection{Experimental Studies on Jet Screech}

Shock cell patterns of imperfectly expanded jets were experimentally observed and photographed as early as 1907 by Prandtl and Meyer (Anderson, 1990). However, the relationship between these patterns and jet noise was first revealed by Powell (1953 a, b). He performed experiments with jets above the critical pressure, and observed the feedback loop that generates the screech noise. He increased the back pressure to obtain a cellular pattern. First, the flow expands on leaving the nozzle, and then contracts to its original diameter, reaching the nozzle exit conditions again and repeating itself. The cycle is dissipated by the turbulent mixing of the stream after several cycles. Shock waves form during the contraction at the end of the cells, growing inwards in the upstream direction from the point of minimum area, forming a conical structure. If the pressure ratio is further increased, a normal shock will form, which removes the apex of the conical shock (later referred to as a Mach disk). Powell describes the sound generated by this underexpanded jet as follows: "While the 'roaring' noise due to turbulent mixing is still present, it may be almost completely dominated by a very powerful noise of completely different character. This may be described as a 'whistle' or 'screech', rather harsh and of a confused nature, becoming much more like a pure note, usually of increased intensity, over certain ranges of pressure ratio."

After Powell's discovery of screech, a lot of visualizations and other experiments have been conducted. Most of the early work was concentrated on time 
averaged measurements while the sound generation is an unsteady process. Westley and Woolley's experiment (1969) was one of the exceptions. They obtained pressure history data for a 2.25 inch diameter jet operating at a pressure ratio of 3.1 and measured a screech frequency of 3170 cycles per second. They also described the interaction of shock waves and disturbances, and observed strong pressure radiation at third and fourth shock cells and release of flow disturbances at the first cell.

Fisher and Morfey (1976) have reviewed each of the three jet noise components. Their analysis about screech reveals a quick formula to predict the frequency, but the amplitude varied widely even during constant operation. A large reflector plate in the nozzle exit plane was observed to eliminate the variation of screech amplitude. Addition of a projection on the nozzle lip eliminated screech tones.

Norum and Seiner (1980) made experiments with jets of Mach number 1, 1.5 and 2 using nozzles of diameters $3.98,4.27,4.99 \mathrm{~cm}$., respectively. They observed that the shock noise peak frequency is dependent on shock cell spacing. Their screech frequencies were about $6-8 \mathrm{kHz}$ for all cases. They conclude that each shock cell generates a different frequency, and downstream cells generate higher frequencies, however, jet mixing noise frequency decreases going downstream.

Raman and Rice (1994) have investigated instability modes excited by screech tones in rectangular jets. They used rectangular nozzles with Mach number of 1.44 and Reynolds number of 96,611 based on smaller dimension of rectangular nozzle. They have generated plots for sound pressure level and phase of screech tones.

Tam, Shen and Raman (1997) have made experiments on screech tones of supersonic jets from bevelled rectangular nozzles at Mach number 1.4. Shock cell structure and frequency pattern for a bevelled nozzle are measured and predicted by a vortex sheet model. 
Panda (1999) investigated screech noise generation in axisymmetric jets at Mach numbers 1.19 and 1.42. He made many important observations. He observed interference between upstream propagating sound wave and downstream propagating hydrodynamic wave, and that standing wavelength is not equal to shock spacing. Moreover, fluctuations and convective velocity of organized vortices matched standing wavelength rather than shock spacing, thus the standing wavelength is observed to be a new length scale. He also suggested some unresolved questions to be investigated, which are:

* What is the exact nature of the feedback loop?

* Where do the effective sound sources exist?

* How do the shocks respond to a periodic train of disturbances?

* How do the sound waves generate more periodic disturbances in the flow?

\subsection{Numerical Studies on Jet Screech}

Cain (1993) made computations on supersonic jet screech. He solved NavierStokes equations in two and three dimensions, at Mach numbers of 1.35 and 1.60 and Reynolds number of 25000 based on nozzle throat. He used central first, second, upwind first, second, third order schemes with characteristic boundary conditions. He made comparisons of these schemes.

Shen and Tam (1998) investigated generation of axisymmetric jet screech tones. They solved Navier-Stokes equations with $k-\varepsilon$ turbulence model in two dimensions. Mach number was selected as 1.00 and 1.25. Tam's radiation and outflow conditions were used. Discretization was done by 7 point DRP scheme with artificial damping.

Casper and Carpenter (1998) compared first and higher order schemes on a model problem with quasi-one-dimensional flow at Mach numbers of 0.8 and 1.8 . They used fourth order ENO scheme with three step Runge-Kutta time integration and prescribed boundary conditions. 
Gribben, Badcock, Richards (2000) have studied shock reflection hysterisis in an underexpanded jet. They solved two-dimensional Navier-Stokes equations at Mach number of 3.0 and Reynolds number of 4000 based on nozzle throat. They used cell centered finite volume method and Osher's scheme with interpolation-extrapolation boundary conditions.

Manning and Lele (2000) have studied sound generation in supersonic jet screech by examining an isolated shear layer. They solved two-dimensional, linearized Euler equations at Reynolds number of 1000 based on shear layer thickness. They compare the results with their previous work where they solved Navier-Stokes equations.

Loh et. al. (2001) have made computations to simulate experiments of Panda (1999). They used unstructured three-dimensional space-time conservation element and solution element method to solve Euler equations. Non-reflecting boundary conditions were used. Their computational domain is exactly simulating Panda's experimental setup. They have computed the shock structure observed in Panda's experiment. 


\section{CHAPTER 3 \\ MATHEMATICAL MODEL}

In this chapter, governing equations, initial and boundary conditions, and turbulence models will be presented for axisymmetric and three-dimensional cases.

\subsection{Axisymmetric model}

We wish to simulate screech noise generation for a supersonic axisymmetric jet. Mach number is chosen as 1.19 for comparison with experiments (Panda, 1999). Governing equations are full Navier-Stokes equations in cylindrical coordinates. Since the problem is axisymmetric, we need only two momentum equations in axial and radial directions $(x, y)$, as well as continuity and energy equations. Large eddy simulation is used as turbulence model, which contributes as an addition to the viscosity. Conservative forms of these equations are used in order to capture shocks appropriately.

In the following vector form of the equations, first row is continuity, second row is energy, third row is $x$-momentum (axial) and fourth row is $y$-momentum (radial) equations:

$$
\begin{gathered}
\frac{\partial U}{\partial t}=-R \\
R=\frac{\partial}{\partial x}(F-F V)+\frac{1}{y} \frac{\partial}{\partial y}\left(G-G_{v}\right)+\frac{S}{y} \\
U=\left\{\begin{array}{c}
\rho E \\
\rho u \\
\rho v
\end{array}\right\} \quad F=\left\{\begin{array}{c}
\rho E u+p u \\
\rho u^{2}+p \\
\rho u v
\end{array}\right\} \quad G=\left\{\begin{array}{c}
\rho E v+p v \\
\rho v u \\
\rho v^{2}+p
\end{array}\right\}
\end{gathered}
$$




$$
F_{v}=\left\{\begin{array}{c}
0 \\
u \tau_{x x}+v \tau_{x y}+k \frac{\partial T}{\partial x} \\
\tau_{x x} \\
\tau_{x y}
\end{array}\right\} \quad G_{v}=\left\{\begin{array}{c}
0 \\
u \tau_{x y}+v \tau_{y y}+k \frac{\partial T}{\partial y} \\
\tau_{x y} \\
\tau_{y y}
\end{array}\right\} \quad S=\left\{\begin{array}{c}
0 \\
0 \\
0 \\
p-\tau_{\theta \theta}
\end{array}\right\}
$$

$$
\begin{aligned}
& \tau_{\mathrm{xx}}=\frac{2}{3} \mu\left(2 \frac{\partial \mathrm{u}}{\partial \mathrm{x}}-\frac{\partial \mathrm{v}}{\partial \mathrm{y}}-\frac{\mathrm{v}}{\mathrm{r}}\right) \\
& \tau_{\mathrm{yy}}=\frac{2}{3} \mu\left(2 \frac{\partial \mathrm{v}}{\partial \mathrm{y}}-\frac{\partial \mathrm{u}}{\partial \mathrm{x}}-\frac{\mathrm{v}}{\mathrm{r}}\right) \\
& \tau_{\mathrm{xy}}=\mu\left(\frac{\partial \mathrm{u}}{\partial \mathrm{y}}+\frac{\partial \mathrm{v}}{\partial \mathrm{x}}\right) \\
& \tau_{\theta \theta}=\frac{2}{3} \mu\left(\frac{2 \mathrm{v}}{\mathrm{r}}-\frac{\partial \mathrm{v}}{\partial \mathrm{y}}-\frac{\partial \mathrm{u}}{\partial \mathrm{x}}\right)
\end{aligned}
$$

\subsubsection{Initial and boundary conditions}

We use the ambient density and pressure to be the scales for dependent variables. Velocity scale is $\sqrt{\mathrm{RT}}$, where $R$ is the gas constant and $T$ is the temperature. We assume that the walls and the ambient are initially at a constant temperature of 300 degree Kelvins. Wall temperature is assumed to be constant throughout the solution. Therefore, the initial ambient conditions are:

$$
\rho_{0}=1 \quad \mathrm{p}_{0}=1 \quad \mathrm{u}_{0}=0 \quad \mathrm{v}_{0}=0
$$

At the nozzle exit, elevated pressure $p=p_{e}$ is imposed to get an underexpanded jet. The flow variables at the exit are given by

$$
\mathrm{p}_{\mathrm{e}}=\mathrm{p}_{0}\left[\frac{1+\frac{1}{2}(\gamma-1) \mathrm{M}_{\mathrm{j}}^{2}}{1+\frac{1}{2}(\gamma-1)}\right]^{\frac{\gamma}{\gamma-1}}
$$




$$
\begin{gathered}
\rho_{e}=\rho_{0} \frac{\gamma+1}{2}\left[\frac{1+\frac{1}{2}(\gamma-1) M_{j}^{2}}{1+\frac{1}{2}(\gamma-1)}\right]^{\frac{\gamma}{\gamma-1}} \\
u_{e}=\sqrt{\frac{2 \gamma}{\gamma+1}}, \quad v_{c}=0
\end{gathered}
$$

For supersonic outflow, all characteristics go out of the domain; therefore the variables are simply extrapolated. For subsonic outflow points, the following characteristic condition is used ${ }^{2}$ :

$$
\left(\frac{1}{\rho c} \frac{\partial p}{\partial x}-\frac{\partial u}{\partial x}\right)(u-c)=0
$$

where $c$ is the local speed of sound and is given by $c=\sqrt{\gamma R T}$.

\subsubsection{Turbulence modeling}

We use a simple large eddy simulation (LES) model called Smagorinsky's subgrid scale model

$$
\mu_{1}=C_{\mathrm{s}}^{2} \Delta \mathrm{x} \Delta \mathrm{y}\left(2 \mathrm{~S}_{\mathrm{ij}} \mathrm{S}_{\mathrm{ij}}\right)^{1,2}
$$

where

$$
S_{i j}=\frac{1}{2}\left(\frac{\partial u_{i}}{\partial x_{j}}+\frac{\partial u_{j}}{\partial x_{i}}\right)
$$

and $C_{s}=0.1$. Then in computation, $\mu_{\mathrm{t}}$ is added to the viscosity $\mu$. 


\subsection{Three-Dimensional Model}

We wish to simulate screech noise generation for a supersonic threedimensional jet. Mach number is chosen as 1.43 for comparison with experiments. Governing equations are full Navier-Stokes equations in cylindrical coordinates. We need three momentum equations in axial, radial and azimuthal directions $(x, y, z)$, as well as continuity and energy equations. This system reduces to the axisymmetric model, when the $\mathrm{z}$ direction derivatives and variables are assumed to be zero. Conservative forms of these equations are used in order to capture shocks appropriately.

In the following vector form of the equations, first row is continuity, second row is energy, third row is x-momentum (axial), fourth row is y-momentum (radial), and fifth row is the z-momentum (azimuthal) equations:

$$
\begin{gathered}
\frac{\partial U}{\partial t}=-R \\
R=\frac{\partial}{\partial x}\left(F-F_{v}\right)+\frac{1}{y} \frac{\partial}{\partial y}\left(G-G_{v}\right)+\frac{S}{y} \\
U=\left\{\begin{array}{c}
\rho \\
\rho E \\
\rho u \\
\rho v \\
\rho w
\end{array}\right\} \quad F=\left\{\begin{array}{c}
\rho E u+p u \\
\rho u^{2}+p \\
\rho u v \\
\rho u w
\end{array}\right\} \\
G=\left\{\begin{array}{c}
\rho u \\
\rho E v+p v \\
\rho v u \\
\rho v^{2}+p \\
\rho v w
\end{array}\right\} \quad H=\left\{\begin{array}{c}
\rho w \\
\rho E w+p w \\
\rho w u \\
\rho w v \\
\rho w^{2}+p
\end{array}\right\}
\end{gathered}
$$




$$
\begin{aligned}
& F_{v}=\left\{\begin{array}{c}
0 \\
u \tau_{x x}+v \tau_{x y}+w \tau_{x z}+k \frac{\partial T}{\partial x} \\
\tau_{x x} \\
\tau_{x y} \\
\tau_{x z}
\end{array}\right\} G_{v}=\left\{\begin{array}{c}
0 \\
u \tau_{x y}+v \tau_{y y}+w \tau_{y z}+k \frac{\partial T}{\partial y} \\
\tau_{x y} \\
\tau_{y y} \\
\tau_{y z}
\end{array}\right\} \\
& H_{v}=\left\{\begin{array}{c}
u \tau_{x z}+v \tau_{y z}+w \tau_{z z}+k \frac{\partial T}{\partial z} \\
\tau_{x z} \\
\tau_{y z} \\
\tau_{z z}
\end{array}\right\} S=\left\{\begin{array}{c}
0 \\
0 \\
0 \\
p+\rho w^{2}-\tau_{\theta \theta} \\
\tau_{y z}-\rho v w
\end{array}\right\}
\end{aligned}
$$

$$
\begin{aligned}
& \tau_{\mathrm{xx}}=\frac{2}{3} \mu\left(2 \frac{\partial \mathrm{u}}{\partial \mathrm{x}}-\frac{\partial \mathrm{v}}{\partial \mathrm{y}}-\frac{1}{\mathrm{y}} \frac{\partial \mathrm{w}}{\partial \mathrm{z}}-\frac{\mathrm{v}}{\mathrm{y}}\right) \\
& \tau_{\mathrm{yy}}=\frac{2}{3} \mu\left(2 \frac{\partial \mathrm{v}}{\partial \mathrm{y}}-\frac{\partial \mathrm{u}}{\partial \mathrm{x}}-\frac{1}{\mathrm{y}} \frac{\partial \mathrm{w}}{\partial \mathrm{z}}-\frac{\mathrm{v}}{\mathrm{y}}\right) \\
& \tau_{0 \theta}=\frac{2}{3} \mu\left(\frac{2}{\mathrm{y}} \frac{\partial \mathrm{w}}{\partial \mathrm{z}}+\frac{2 \mathrm{v}}{\mathrm{y}}-\frac{\partial \mathrm{v}}{\partial \mathrm{y}}-\frac{\partial \mathrm{u}}{\partial \mathrm{x}}\right) \\
& \tau_{\mathrm{xy}}=\mu\left(\frac{\partial \mathrm{u}}{\partial \mathrm{y}}+\frac{\partial \mathrm{v}}{\partial \mathrm{x}}\right) \\
& \tau_{\mathrm{xz}}=\mu\left(\frac{1}{\mathrm{y}} \frac{\partial \mathrm{u}}{\partial \mathrm{z}}+\frac{\partial \mathrm{w}}{\partial \mathrm{x}}\right) \\
& \tau_{\mathrm{yz}}=\mu\left(\frac{1}{\mathrm{y}} \frac{\partial \mathrm{v}}{\partial \mathrm{z}}-\frac{\mathrm{w}}{\mathrm{y}}+\frac{\partial \mathrm{w}}{\partial \mathrm{y}}\right)
\end{aligned}
$$

All initial and boundary conditions in radial and axial terms are the same as in axisymmetric case, and azimuthal terms are all zero where applicable. For example. the initial conditions are same with addition of $w$ being zero.

$$
\rho_{0}=1 \quad p_{0}=1 \quad u_{0}=0 \quad v_{0}=0 \quad w_{0}=0
$$

Nozzle exit conditions are same as well: $p_{e}, \rho_{e}, u_{s}, v_{e}$ are given by (3.6)-(3.8). 


$$
\mathrm{w}_{\mathrm{c}}=0
$$

is the only additional boundary condition. The characteristic outflow condition is also same as (3.9).

We used large eddy simulation in the axisymmetric case, but for threedimensional computations, we had to eliminate turbulence modeling in order to save computational resources.

\subsubsection{Periodic conditions}

The solution of helical mode is symmetric along the origin, so it is necessary only to model half of the cylinder in the azimuthal direction. We set two ghost points for each side and set every variable of them equal to the points straight across, as in Fig. 2.

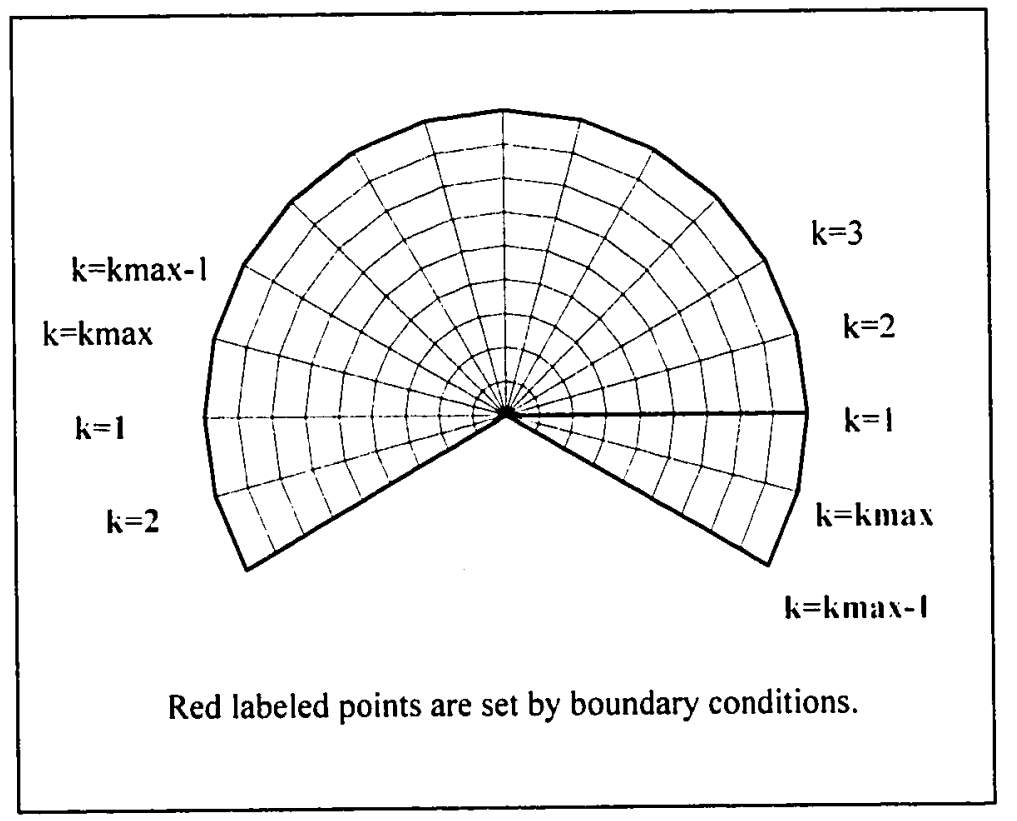

Figure 2: Ghost points for periodic boundary condition. 


\section{CHAPTER 4 \\ COMPUTATIONAL METHOD}

In this chapter, the computational schemes used in this study will be introduced and their advantages over other schemes will be discussed.

Weighted essentially non-oscillatory (WENO) scheme of fifth order is used for spatial discretizations. It is an advanced form of essentially non-oscillatory (ENO) scheme, where, at each point, weighted average of all possible stencils is used instead of just picking the best one.

Temporal discretization used in this study is third order total variation diminishing (TVD) scheme. TVD schemes have the advantage of preventing overshoot and oscillations. Third order is the highest order that does not need the computation of an adjoint operator, which is a very costly computation.

\subsection{Development of ENO and WENO schemes}

Finite difference and finite volume schemes are based on interpolations of discrete data using polynomials or other simple functions. A wider stencil will increase the accuracy of the interpolation as long as the function being interpolated is smooth inside the stencil. Traditional finite difference methods are based on fixed stencil approximations. For example, to obtain a third order accurate interpolation for cell $i$, the information in the cells $i-l, i$, and $i+l$ can be used to build a second order interpolation polynomial. One always looks one cell to the left, one cell to the right and the cell itself, for all points in the domain. This works well for globally smooth problems. However, fixed stencil interpolation of second or higher order accuracy is necessarily oscillatory near a discontinuity. Such oscillations are called "the Gibbs phenomena" and do not decay in magnitude with refined mesh. This often leads to numerical instabilities in nonlinear problems containing discontinuities.

Before 1987, there were mainly two common ways to eliminate or reduce spurious oscillations near discontinuities. One way was to add an artificial viscosity. This could be tuned so that it was large enough at the discontinuity to suppress the 
oscillations, and small elsewhere to maintain high order accuracy. The disadvantage of this method is that fine tuning parameters for artificial viscosity are problem dependent. The second way was to apply limiters to eliminate oscillations. The order of accuracy of the interpolation near the discontinuity was reduced to linear near the shock. TVD (total variation diminishing) property could be achieved for nonlinear scalar one-dimensional problems. The disadvantage of this method is that accuracy degenerates to first order near smooth extrema.

The ENO idea was proposed in the classic paper of Harten, Engquist, Osher and Chakravarthy in 1987. This seems to be the first successful attempt to obtain a self-similar (i.e. no mesh size dependent parameter), uniformly high order accurate, yet essentially non-oscillatory interpolation for piecewise smooth functions. They developed a hierarchy that begins with one or two cells, and then adds one cell at a time to the stencil from the two candidates on the left or right, based on the size of the two relevant Newton divided differences. It is seen in their numerical examples and in later papers, that ENO schemes are indeed uniformly high order accurate and resolve shocks with sharp and monotone transitions. ENO schemes are especially suitable for problems containing both shocks and complicated smooth flow structures, such as those occurring in shock interactions with a turbulent flow and shock interaction with vortices.

After the publication of the original paper of Harten, Engquist, Osher and Chakravarthy in 1987, the original authors and many other researchers have followed the pioneer work, improving the methodology and expanding the area of its applications. Shu and Osher $(1988,1989)$ developed ENO schemes based on point values and TVD Runge-Kutta discretizations, which can reduce computational cost significantly for multi space dimensions. Later, biasing in the stencil choosing process to enhance stability and accuracy were developed in Fatemi (1991) and Shu (1990). Weighted ENO (WENO) schemes were developed, using a convex combination of all candidate stencils instead of just one in the original ENO, Liu (1994), Jiang (1996). ENO schemes based on other than polynomial building blocks were constructed in Iske (1996), Christofi (1995). Sub-cell resolution and artificial compression to sharpen contact discontinuities were studied in Harten (1989), Yang 
(1990), Shu (1989), and Jiang (1996). Multidimensional ENO schemes based on general triangulation were developed in Abgrall (1994). Combination of ENO with multi-resolution ideas was pursued in Bihari (1995). Combination of ENO with spectral method using a domain decomposition approach was carried out in Cai (1993). On the application side, ENO and WENO have been successfully used to simulate shock-turbulence interactions in Shu (1989), Shu (1992), Adarns (1996); to the direct simulation of compressible turbulence in Shu (1992), Walsteijn (1994), Ladeinde (1995); to relativistic hydrodynamics equations in Dolezal (1995); to shock vortex interactions and other gas dynamics problems in Casper (1993), Erlebacher (1997), Jiang (1996); to incompressible flow problems in W. E. Shu (1994), Harabetian (1996); as well as other fields. Most of the problems solved by ENO and WENO schemes contain both strong shocks and rich smooth region structures. Lower order methods usually have difficulties for such problems so it is attractive and efficient to use high order stable methods such as ENO and WENO to handle them.

\subsubsection{Reconstruction and approximation in $1 \mathrm{D}$}

In this section, interpolation and approximation in one space dimension will be examined.

Given a grid

$$
\mathrm{a}=\mathrm{x}_{\frac{1}{2}}<\mathrm{x}_{\frac{3}{2}}<\cdots<\mathrm{x}_{\mathrm{N}-\frac{1}{2}}<\mathrm{x}_{\mathrm{N}+\frac{1}{2}}=\mathrm{b}
$$

cells, cell centers and cell sizes are defined by

$$
\begin{aligned}
& I_{i} \equiv\left[x_{i-\frac{1}{2}}, x_{i+\frac{1}{2}}\right], x_{i} \equiv \frac{1}{2}\left(x_{i-\frac{1}{2}}+x_{i+\frac{1}{2}}\right), \\
& \Delta x_{i} \equiv x_{i+\frac{1}{2}}-x_{i-\frac{1}{2}}, \quad i=1,2, \ldots, N
\end{aligned}
$$

Maximum cell size is donated by 


$$
\Delta \mathrm{x} \equiv \max _{1 \leq \mathrm{i} \leq \mathrm{N}} \Delta \mathrm{x}_{\mathrm{i}}
$$

\subsubsection{One Dimensional Reconstruction}

Given the cell averages of the function $\mathrm{v}(\mathrm{x})$ :

$$
\bar{v}_{i} \equiv \frac{1}{\Delta x_{i}} \int_{1+\frac{1}{2}}^{x+\frac{1}{2}} v(\xi) d \xi, \quad i=1,2, \ldots, N
$$

we want to find a polynomial $p_{i}(x)$, of degree at most $k-1$, for each cell $I_{i}$, such that it is a $\mathrm{k}$-th order accurate approximation to the function $\mathrm{v}(\mathrm{x})$ inside $\mathrm{I}_{\mathrm{i}}$ :

$$
p_{i}(x)=v(x)+O\left(\Delta x^{k}\right) \quad x \in I_{i}, \quad i=1, \ldots, N
$$

In particular, this gives approximations to the function $v(x)$ at the cell boundaries

$$
v_{i+\frac{1}{2}}^{-}=p_{i}\left(x_{i+\frac{1}{2}}\right), \quad v_{i-\frac{1}{2}}^{+}=p_{i}\left(x_{i-\frac{1}{2}}\right), \quad i=1, \ldots, N
$$

which are k-th order accurate:

$$
v_{i+\frac{1}{2}}^{-}=v\left(x_{i+\frac{1}{2}}\right)+O\left(\Delta x^{k}\right), \quad v_{i-\frac{1}{2}}^{+}=v\left(x_{i-\frac{1}{2}}\right)+O\left(\Delta x^{k}\right), \quad i=1, \ldots, N
$$

The polynomial $p_{i}(x)$ can be replaced by other simple functions, such as trigonometric polynomials. It will be assumed that boundary conditions are specified, therefore $\bar{v}_{i}$ is also available for $i \leq 0$ and $i>N$ if needed. Here is a procedure to solve the problem:

Given the location $I_{i}$ and the order of accuracy $k$, we first choose a "stencil", based on $r$ cells to the left, $s$ cells to the right, and $I_{i}$ itself if $r, s \geq 0$, with $r+s+1=k$ : 


$$
S(i) \equiv\left\{I_{i-r}, \ldots, I_{i+s}\right\} .
$$

There is a unique polynomial of degree at most $k-1=r+s$, denoted by $p(x)$ (subscript $i$ will be dropped when there is no confusion), whose cell average in each of the cells in S(i) agrees with that of $\mathrm{v}(\mathrm{x})$ :

$$
\int_{\substack{1+\frac{1}{2} \\ 1}}^{x} p(\xi) d \xi=\bar{v}_{j}, \quad j=i-r, \ldots, i+s .
$$

This polynomial $\mathrm{p}(\mathrm{x})$ is the desired $\mathrm{k}$-th order approximation as long as the function $v(x)$ is smooth in the region covered by the stencil $S(i)$.

For solving the problem, approximations to the values of $v(x)$ at the cell boundaries is also needed. Since the mappings from the given cell averages $\bar{v}_{j}$ in the stencil $\mathrm{S}(\mathrm{i})$ to the values $\mathrm{v}_{i+\frac{1}{2}}^{-}$and $\mathrm{v}_{i-\frac{1}{2}}^{+}$in (4.6) are linear, there exist constants $\mathrm{c}_{\mathrm{ij}}$ and $\widetilde{c}_{r j}$, which depend on the left shift $r$ of the stencil $S(i)$ in (4.8), on the accuracy $k$, and on the cell sizes $\Delta x_{j}$ in the stencil $S(i)$, but not on the function $v$ itself, such that

$$
v_{i+\frac{1}{2}}^{-}=\sum_{j=0}^{k-1} c_{r j} \bar{v}_{i-r+j}, \quad v_{i-\frac{1}{2}}^{+}=\sum_{j=0}^{k-1} \widetilde{c}_{r j} \bar{v}_{i-r+j}
$$

It should be noted that the difference between the values with superscripts \pm at the same location $x_{i+\frac{1}{2}}$ is due to the possibility of different stencils for cell $I_{i}$ and for cell $I_{i+1}$. If the left shift $r$ is identified with the point of reconstruction $x_{i+\frac{1}{2}}$ instead of the cell $I_{i}$, using the stencil (4.8), then the superscripts \pm can be dropped and $\widetilde{c}_{r j}$ need not be considered, since it is clear that $\widetilde{c}_{r j}=c_{r-1, j}$. 
Summarizing the procedure, given the $k$ cell averages $\bar{v}_{i-r}, \ldots, \bar{v}_{i-r+k-1}$,

there are constants $c_{i j}$ such that the reconstructed value at the cell boundary $x_{i+\frac{1}{2}}$ :

$$
v_{i+\frac{1}{2}}=\sum_{j=0}^{k-1} c_{i j} \bar{v}_{i-r+j}
$$

is $\mathrm{k}$-th order accurate:

$$
v_{i+\frac{1}{2}}=v\left(x_{i+\frac{1}{2}}\right)+O\left(\Delta x^{k}\right)
$$

The method to obtain constants $c_{r j}$ is given in Shu (1997). For a uniform grid, $\Delta \mathrm{x}_{\mathrm{i}}=\Delta \mathrm{x}$, the expression for $\mathrm{c}_{\mathrm{rj}}$ is given as

$$
c_{\mathrm{rj}}=\sum_{\mathrm{m}=\mathrm{j}+\mathrm{l}}^{k} \frac{\sum_{\substack{\mathrm{l} \neq 0 \\ l \neq m}}^{k} \prod_{\substack{\mathrm{q} \neq \neq \mathrm{m}, 1 \\ \mathrm{q}=0}}^{\mathrm{k}}(\mathrm{r}-\mathrm{q}+1)}{\prod_{\substack{l=0 \\ l \neq m}}^{k}(m-1)}
$$

Constants $c_{r j}$ for uniform grid for order of accuracy between $k=1$ and $k=5$ are listed in Table 1. 
Table 1: ENO Coefficients

\begin{tabular}{|c|c|c|c|c|c|c|}
\hline$k$ & $\mathrm{r}$ & $\mathrm{j}=0$ & $\mathrm{j}=1$ & $\mathrm{j}=2$ & $\mathrm{j}=3$ & $\mathrm{j}=4$ \\
\hline 1 & -1 & 1 & & & & \\
\hline & 0 & 1 & & & & \\
\hline \multirow{5}{*}{2} & -1 & $3 / 2$ & $-1 / 2$ & & & \\
\hline & 0 & $1 / 2$ & $1 / 2$ & & & \\
\hline 1 & $-1 / 2$ & $3 / 2$ & & & \\
\hline 3 & -1 & $11 / 6$ & $-7 / 6$ & $1 / 3$ & & \\
\hline 0 & $1 / 3$ & $5 / 6$ & $-1 / 6$ & & \\
\hline 1 & $-1 / 6$ & $5 / 6$ & $1 / 3$ & & \\
\hline 2 & $1 / 3$ & $-7 / 6$ & $11 / 6$ & & \\
\hline & -1 & $25 / 12$ & $-23 / 12$ & $13 / 12$ & $-1 / 4$ & \\
\hline 0 & $1 / 4$ & $13 / 12$ & $-5 / 12$ & $1 / 12$ & \\
\hline 1 & $-1 / 12$ & $7 / 12$ & $7 / 12$ & $-1 / 12$ & \\
\hline 2 & $1 / 12$ & $-5 / 12$ & $13 / 12$ & $1 / 4$ & \\
\hline 3 & $-1 / 4$ & $13 / 12$ & $-23 / 12$ & $25 / 12$ & \\
\hline-1 & $137 / 60$ & $-163 / 60$ & $137 / 60$ & $-21 / 20$ & $1 / 5$ \\
\hline 0 & $1 / 5$ & $77 / 60$ & $-43 / 60$ & $17 / 60$ & $1 / 20$ \\
\hline 1 & $-1 / 20$ & $9 / 20$ & $47 / 60$ & $-13 / 60$ & $1 / 30$ \\
\hline 2 & $1 / 30$ & $-13 / 60$ & $47 / 60$ & $9 / 20$ & $-1 / 20$ \\
\hline 3 & $-1 / 20$ & $17 / 60$ & $-43 / 60$ & $77 / 60$ & $1 / 5$ \\
\hline 4 & $1 / 5$ & $-21 / 20$ & $137 / 60$ & $-163 / 60$ & $137 / 60$ \\
\hline
\end{tabular}


From Table 1, it could be obtained, for example, that

$$
v_{i+\frac{1}{2}}=-\frac{1}{6} \bar{v}_{i-1}+\frac{5}{6} \bar{v}_{i}+\frac{1}{3} \bar{v}_{i+1}+O\left(\Delta x^{3}\right)
$$

\subsubsection{One Dimensional Conservative approximation}

Let $\mathrm{v}(\mathrm{x})$ be a function whose point values are known:

$$
\mathrm{v}_{\mathrm{i}} \equiv \mathrm{v}\left(\mathrm{x}_{\mathrm{i}}\right)
$$

A numerical flux function is needed

$$
\hat{\mathrm{v}}_{\mathrm{i}+\frac{1}{2}} \equiv \hat{\mathrm{v}}\left(\mathrm{v}_{\mathrm{i}-\mathrm{r}}, \ldots, \mathrm{v}_{\mathrm{i}+\mathrm{s}}\right), \quad \mathrm{i}=0,1, \ldots, \mathrm{N}
$$

such that the flux difference approximates the derivative $\mathrm{v}^{\prime}(\mathrm{k})$ to $\mathrm{k}$-th order accuracy:

$$
\frac{1}{\Delta x_{i}}\left(\hat{v}_{i+\frac{1}{2}}-\hat{v}_{i-\frac{1}{2}}\right)=v^{\prime}\left(x_{i}\right)+O\left(\Delta x^{k}\right)
$$

All $v_{i}$ are assumed to be available for $i \leq 0$ and $i>N$ if needed.

The solution of this problem is necessary for high order conservative finite difference schemes based on point values rather than finite volume schemes based on cell averages. This problem is closely related to the problem of one-dimensional reconstruction in the previous section.

Assuming a uniform grid $\Delta \mathrm{x}_{\mathrm{i}}=\Delta \mathrm{x}$, if a function $\mathrm{h}(\mathrm{x})$ can be found such that 


$$
v(x)=\frac{1}{\Delta x} \int_{x-\frac{\Delta x}{2}}^{x+\frac{\Delta x}{2}} h(\xi) d \xi
$$

then the derivative is

$$
\mathrm{v}^{\prime}(\mathrm{x})=\frac{1}{\Delta \mathrm{x}}\left[\mathrm{h}\left(\mathrm{x}+\frac{\Delta \mathrm{x}}{2}\right)-\mathrm{h}\left(\mathrm{x}-\frac{\Delta \mathrm{x}}{2}\right)\right]
$$

To achieve (4.16), $\mathrm{h}(\mathrm{x})$ can be used as the flux function

$$
\hat{\mathrm{v}}_{\mathrm{i}+\frac{1}{2}}=h\left(\mathrm{x}_{i+\frac{1}{2}}\right)+\mathrm{O}\left(\Delta \mathrm{x}^{\mathrm{k}}\right)
$$

Noting that the known function $v(x)$ is the cell average of the unknown function $h(x)$, the reconstruction procedure of the previous section can be used to obtain

$$
\hat{v}_{i+\frac{1}{2}}=\sum_{j=0}^{k-1} c_{r j} v_{i-r+i},
$$

where the constants $\left\{c_{\mathrm{rj}}\right\}$ are given by (4.13) and table 1 .

\subsection{Spatial Discretization}

We use weighted essentially non-oscillatory (WENO) scheme for spatial discretization. This is an improved version of essentially non-oscillatory (ENO) scheme that takes a weighted average of all possible stencils ${ }^{7}$.

One-dimensional conservative ENO approximation to the derivative of a function $v(x)$, whose point values $\mathrm{v}_{\mathrm{i}} \equiv \mathrm{v}\left(\mathrm{x}_{\mathrm{i}}\right)$ are known, to the $k^{\text {sh }}$ order, is given by 


$$
\frac{1}{\Delta x}\left(\hat{v}_{i+\frac{1}{2}}-\hat{v}_{i-\frac{1}{2}}\right)=v^{\prime}\left(x_{i}\right)+O\left(\Delta x^{k}\right)
$$

where $\hat{v}$ are the flux functions:

$$
\hat{v}_{i+\frac{1}{2}}=\sum_{j=0}^{k-1} c_{r j} v_{i-r+j}
$$

Constants $\left\{c_{r j}\right\}$ are given in (Shu, 1997). The stencil can be selected by varying the shift parameter $r$.

ENO scheme is high order accurate and non-oscillatory for piecewise smooth functions, and does not need any artificial viscosity. However, discontinuities like shocks will reduce the accuracy of the stencil, so they should be avoided. There are several methods to select the stencil such that the discontinuity is avoided ${ }^{7}$. These methods cover $2 k-l$ cells to choose one of the candidate stencils and obtain $k^{\text {th }}$ order accuracy. WENO uses a weighted average of all possible stencils and the accuracy increases to $(2 k-1)^{\text {th }}$ order.

Let the candidate stencils be

$$
v_{r \cdot \frac{1}{2}}^{(r)}=\sum_{j=0}^{k-1} c_{r j} \bar{v}_{i-r+j} \quad r=0, \ldots, k-1
$$

WENO will take a combination of all $v_{1, !}^{(r)}$ in $(7)$ :

$$
v_{r+\frac{1}{2}}=\sum_{j=0}^{k-1} \omega_{r} v^{r}
$$

For stability and consistency, all $\omega_{\mathrm{r}}$ must be positive and their sum must be equal to 1 . 
Choice of weights $\omega_{\mathrm{r}}$ is crucial for success of WENO. The weight should approach zero for stencils that have a discontinuity. We use the following selection criteria in Shu's lecture notes:

$$
\begin{gathered}
\omega_{r}=\frac{\alpha_{r}}{\sum_{s=0}^{k-1} \alpha_{s}} \\
\alpha_{r}=\frac{d_{r}}{\left(\varepsilon+\beta_{r}\right)^{2}}
\end{gathered}
$$

Here, $\varepsilon$ is a small number used to avoid division by zero, which we choose as $10^{-6} . \beta_{r}$ are called "smooth indicators" of a stencil. For $k=3$, corresponding to $5^{\text {th }}$ order WENO scheme, the smooth indicators are given by

$$
\begin{aligned}
& \beta_{0}=\frac{13}{12}\left(\bar{v}_{i}-2 \bar{v}_{i+1}+\bar{v}_{i+2}\right)^{2}+\frac{1}{4}\left(3 \bar{v}_{i}-4 \bar{v}_{i+1}+\bar{v}_{i+2}\right)^{2} \\
& \beta_{1}=\frac{13}{12}\left(\bar{v}_{i-1}-2 \bar{v}_{i}+\bar{v}_{i+1}\right)^{2}+\frac{1}{4}\left(\bar{v}_{i-1}+\bar{v}_{i+1}\right)^{2} \\
& \beta_{2}=\frac{13}{12}\left(\bar{v}_{i-2}-2 \bar{v}_{i-1}+\bar{v}_{i}\right)^{2}+\frac{1}{4}\left(\bar{v}_{i-2}-4 \bar{v}_{i-1}+3 \bar{v}_{i}\right)^{2}
\end{aligned}
$$

Using these smooth indicators to calculate the weights, we get $5^{\text {th }}$ order WENO scheme. We use density to calculate smooth indicators at each point, and use it for all five variables. 


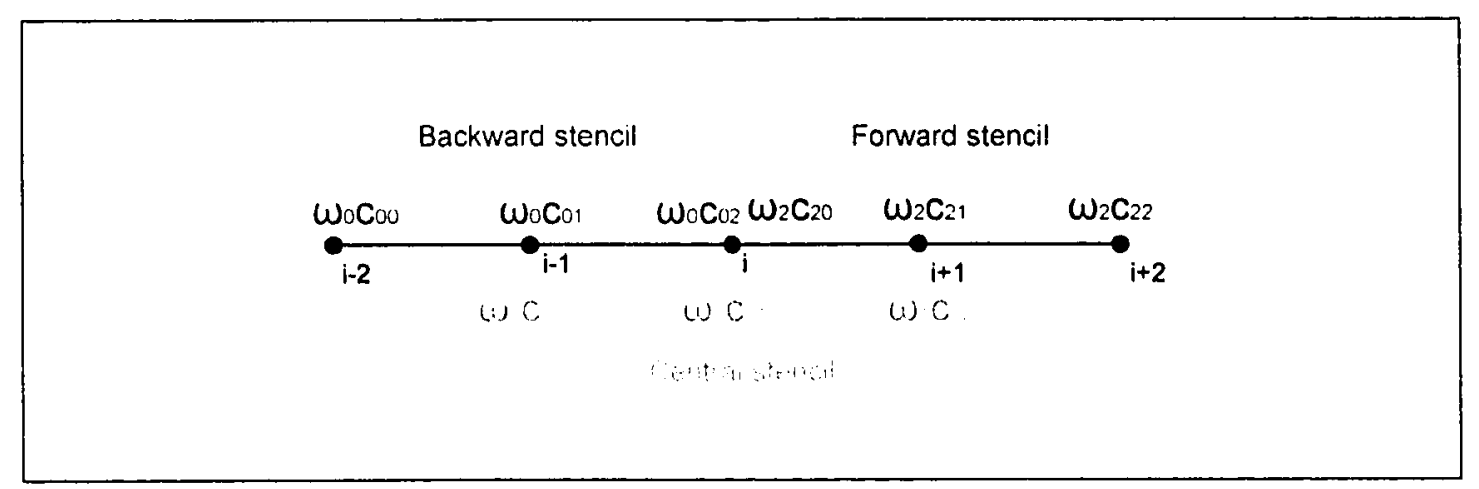

Figure 3: Fifth order WENO discretization

\subsection{Temporal Discretization}

Temporal discretization is done by a third order total variation diminishing (TVD) Runge-Kutta scheme suggested in Shu (1997). TVD schemes are preferred because they prevent oscillation and overshooting at discontinuities. Beyond third order, TVD schemes involve adjoint operators and storage requirement increases rapidly.

Temporal discretization used in this work is the following:

$$
\begin{aligned}
& u^{(1)}=u^{n}+\Delta t L\left(u^{n}\right) \\
& u^{(2)}=\frac{3}{4} u^{n}+\frac{1}{4} u^{(1)}+\frac{1}{4} \Delta t L\left(u^{(1)}\right) \\
& u^{n+1}=\frac{1}{3} u^{n}+\frac{2}{3} u^{(2)}+\frac{2}{3} \Delta t L\left(u^{(2)}\right)
\end{aligned}
$$

where $L(u)$ is the residual of spatial discretization of flux vector $u$.

ENO version of this discretization was used by Atkins (1991) on benchmark problems and found to be very accurate and effective for shock capturing. 


\subsection{Two-Dimensional Grid Generation}

The domain for 2-dimensional computations is 12 diameters long in the axial direction and 8 diameters long in the radial direction; the nozzle wall is from $x=0$ to $x=4, y=0.5$ to $y=0.75$, as in Fig. 4 . The geometry is very simple and rectangular, so $\mathrm{x}$ and $\mathrm{y}$ directions can be handled separately. The nozzle lip is the most sensitive area because a shear layer and strong shock is expected; therefore, more points are needed there. Fewer points are needed towards the right and top ends of the domain, where ambient conditions are expected. At the nozzle lip, the grid must be close to square, that is, $x$ and $y$ increments must be of same order, in order to achieve convergence in that critical area. There is no such requirement at the far sides of the domain. The physical domain of $x-y$ plane can be mapped to a uniform grid computational domain of $\zeta-\eta$ such that $x=x(\zeta)$ and $y=y(\eta)$ are polynomials. Then, coefficients can be selected to satisfy boundary conditions and desired point distribution. In the $\mathrm{x}$-direction, a $3^{\text {rd }}$ order polynomial is selected such as:

$$
\mathrm{x}(\zeta)=\mathrm{A} \zeta^{3}+\mathrm{B} \zeta^{2}+\mathrm{C} \zeta+\mathrm{D}
$$

There are 2 boundary conditions that must be satisfied:

$$
\mathrm{x}(0)=0 \quad \mathrm{x}(1)=\mathrm{W}=12
$$

Since there are 4 coefficients, 2 more conditions can be assigned liberally to obtain a good distribution. Since the minimum increment is desired to be at the nozzle lip $(x=$ $x_{n}=4$ ), the derivative of $x$ at that point should be minimum. Therefore, the second derivative can be set to 0 at the nozzle lip to ensure minimum grid size there. However, the $\zeta$ value at that point is also needed to do that assignment. Let that value be $p$, such that

$$
x(p)=x_{n}=4
$$


and

$$
\left.\frac{\mathrm{d}^{2} \mathrm{x}}{\mathrm{d} \zeta^{2}}\right|_{\zeta=p}=0
$$

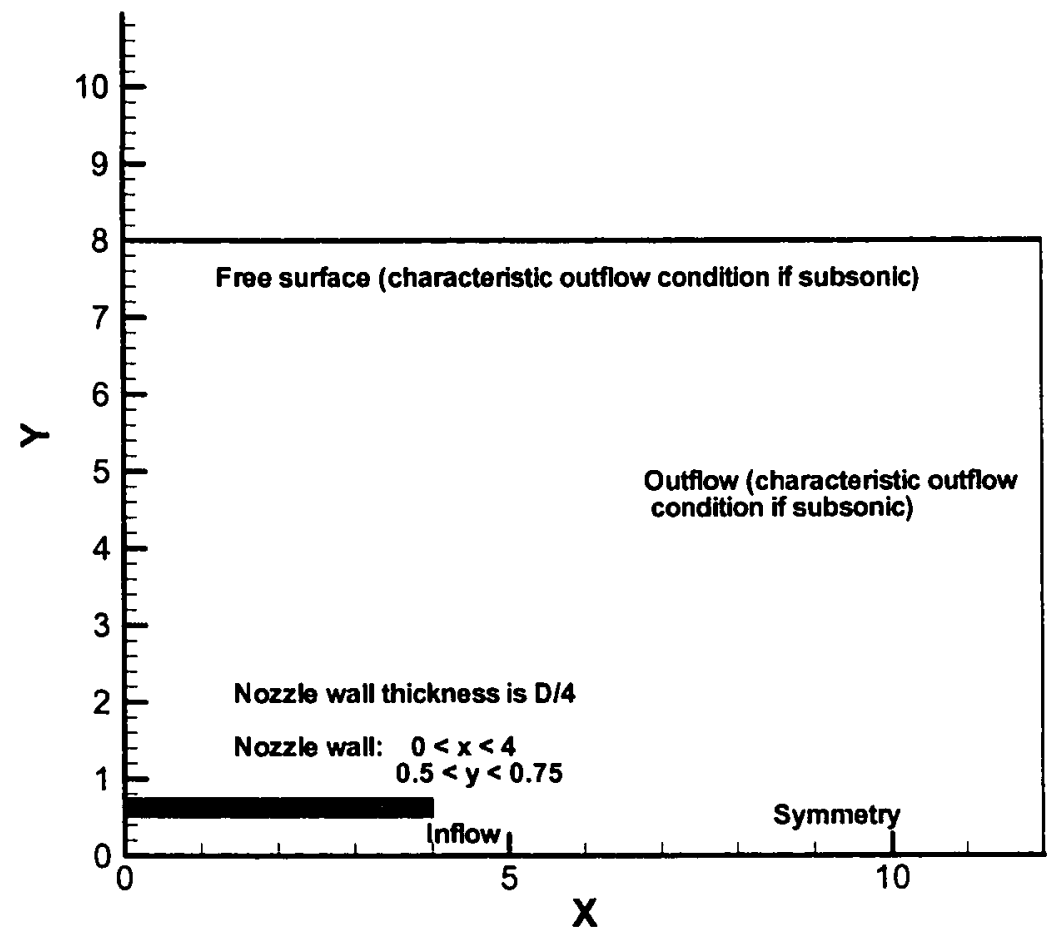

Figure 4: Computational domain and boundary conditions

Here $p$ is the ratio of number of points that are on the left of nozzle lip to number of total points; and it can be assigned freely, as long as the metrics are positive. The conditions (4.31) and (4.32) complete the number of total conditions to 4. For any given $\mathrm{p}$, the solution is 


$$
\left[\begin{array}{l}
A \\
B \\
C \\
D
\end{array}\right]=\left[\begin{array}{cccc}
1 & 1 & 1 & 1 \\
0 & 0 & 0 & 1 \\
p^{3} & p^{2} & p & 1 \\
6 p & 2 & 0 & 0
\end{array}\right]^{-1}\left[\begin{array}{c}
W \\
0 \\
x_{n} \\
0
\end{array}\right]=\frac{1}{\left(-2 p^{3}+3 p^{2}-p\right)}\left[\begin{array}{c}
-p W+x_{n} \\
3 p^{2} W-3 p x_{n} \\
-2 p^{3} W+(3 p-1) x_{n} \\
0
\end{array}\right]
$$

The value for $\mathrm{p}$ is chosen as 0.44 for this computation, which gives the coefficients A, B, C values of $43.290,-57.143$, and 25.853 , respectively.

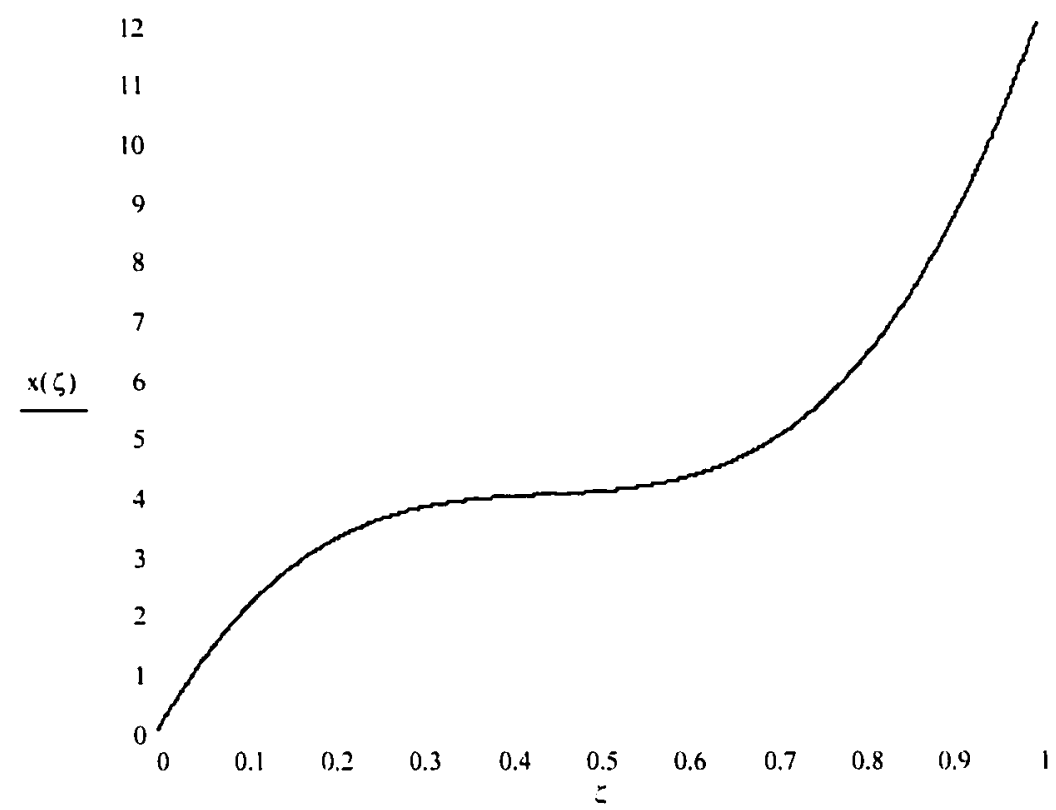

Figure 5 Grid generation function for $\mathrm{x}$-direction

Similar study can be made in the y-direction with a symmetrical distribution about the mid point of the nozzle wall to ensure equal grid sizes in both corners of the nozzle wall. A new axis can be defined as $y^{\prime}$ from the mid point of the nozzle, $y=$ 0.625 . The computational domain variable $\eta$ is also replaced with $\eta^{\prime}$ that runs from 0 to 1 while $y^{\prime}$ varies from 0 to maximum. The function $y^{\prime}\left(\eta^{\prime}\right)$ must be odd to satisfy symmetry about the new axis. There are 2 boundary conditions and 2 points in the domain, wall start and end, to adjust the distribution.

$$
y^{\prime}\left(\eta^{\prime}\right)=A \eta^{\prime 5}+B \eta^{\prime 3}+C \eta^{\prime}
$$


The boundary condition $y^{\prime}(0)=0$ is automatically satisfied by this form. The other boundary condition is at the upper end:

$$
y^{\prime}(1)=H^{\prime}=8-0.625=7.375
$$

Similar to the $\mathrm{p}$ above, this time $\mathrm{p}_{1}$ and $\mathrm{p}_{2}$ are defined to be the values at nozzle ends, such that

$$
y^{\prime}\left(p_{1}\right)=y_{n 1}=0.125
$$

and

$$
y^{\prime}\left(p_{2}\right)=y_{n 2}=0.625
$$

The $y_{n 2}$ value is the mirror image of the origin $y=0$ and is included to control the number of points between the origin and the walls, and to ensure that the wall corners lie on a grid point. Again, $p_{1}$ and $p_{2}$ are freely chosen as long as the metrics are positive. The solution for any $\mathrm{p} 1$ and $\mathrm{p}_{2}$ is given by

$$
\left[\begin{array}{l}
A \\
B \\
C
\end{array}\right]=\left[\begin{array}{ccc}
1 & 1 & 1 \\
p_{1}^{5} & p_{1}^{3} & p_{1} \\
p_{2}^{5} & p_{2}^{3} & p_{2}
\end{array}\right]^{-1}\left[\begin{array}{l}
H^{\prime} \\
y_{n 1} \\
y_{n 2}
\end{array}\right]
$$

The chosen values for $\mathrm{p}_{1}$ and $\mathrm{p}_{2}$ are 0.2 and 0.6 respectively, yielding the values of $\mathrm{A}, \mathrm{B}, \mathrm{C}$ as $8.952,-2.279$, and 0.7018 . Substituting original variables $\mathrm{y}$ and $\eta$, the solution takes the form:

$$
y(\eta)=A\left(\left(1+p_{2}\right) \eta-p_{2}\right)^{5}+B\left(\left(1+p_{2}\right) \eta-p_{2}\right)^{3}+C\left(\left(1+p_{2}\right) \eta-p_{2}\right)+y_{n 2}
$$




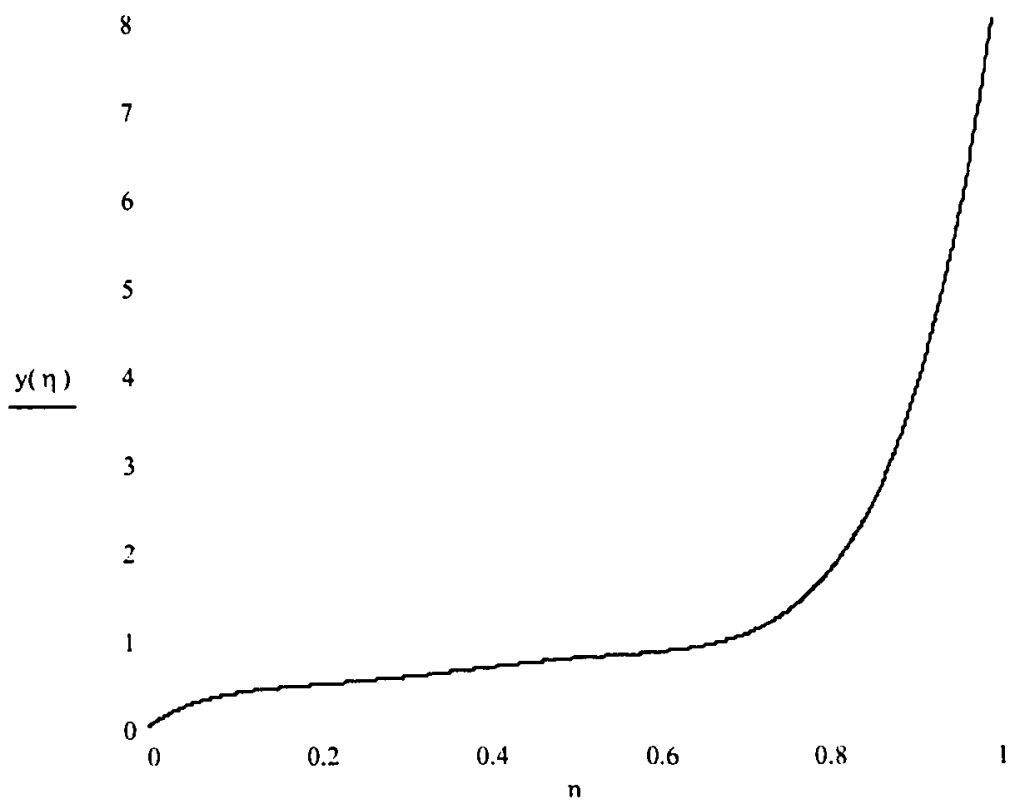

Figure 6 Grid generation function in the y-direction.

The 2-dimensional grid generated by these formulas looks like the following: ( 1 of every 5 lines shown)

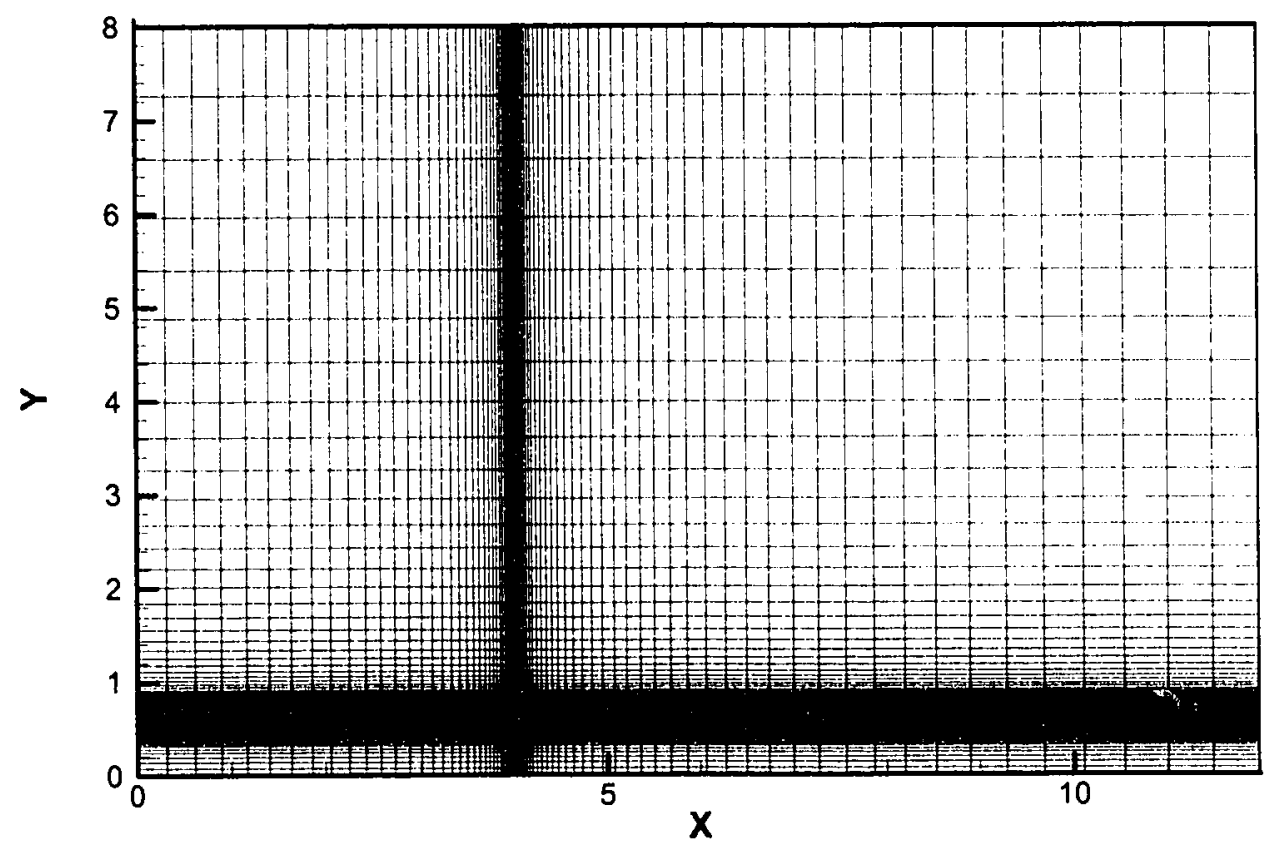

Figure 7: Two-dimensional grid. 


\subsection{Three-Dimensional Grid Generation}

For three-dimensional simulation, we select a domain of 8 diameters long in the axial direction, 4 diameters long in the radial direction and a semi circle in the azimuthal direction as in Fig. 8. Because of symmetry, modeling half of the domain in the azimuthal direction is sufficient. The flow inside the jet nozzle is a choked flow while outside the nozzle, jet Mach number is $\mathrm{M}_{\mathrm{j}}=1.43$, and the ambient flow is stationary. These conditions correspond to the experimental setup of Panda. We had to use a smaller domain than our axisymmetric study, because three-dimensional simulation requires more computational resources. There are 200 grid points in $x$ direction and 150 grid points in y direction. The nozzle extends 2 diameters into the domain in order to show backward propagating screech waves. The inflow plane is 3 cells behind the nozzle exit so that instability waves can develop easily at the nozzle exit. The nozzle wall thickness is $\mathrm{D} / 4$, as in the experiment. The grid is rectangular, with more points close to nozzle exit in $\mathrm{x}$-direction and nozzle wall in y-direction. Polynomials are used to transform from uniform grid to general coordinates, so the metrics are easily obtained analytically. It has been observed that the grid size must be approximately same in each direction (square grid) at the corners of the nozzle wall; otherwise, the solution at these points will diverge. In the azimuthal direction, the semi-circle is divided into 16 equally spaced slices. 


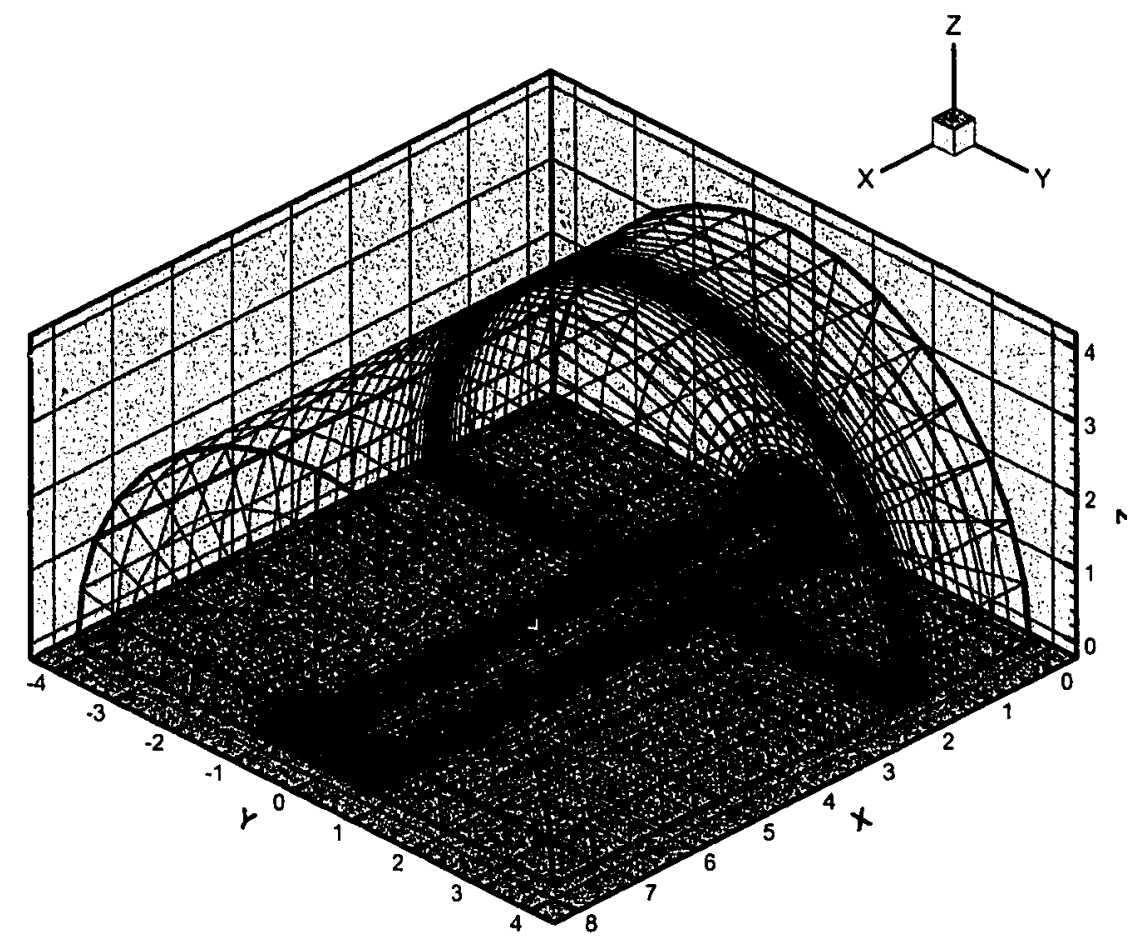

Figure 8: Computational domain and grid for 3-D simulation. 


\section{CHAPTER 5 \\ TWO-DIMENSIONAL SIMULATION RESULTS}

\subsection{Introduction}

Shock cells will develop in an imperfectly expanded supersonic jet. These cells will react with perturbations and generate screech tones, which will produce further perturbations and therefore form a feedback loop. Screech noise is generated as the shock cell structure interacts with disturbances. Panda (1999) conducted experiments to observe the screech phenomenon. He observed that there was interference between upstream propagating sound wave and downstream propagating hydrodynamic wave, and that the fluctuations and convective velocity of organized vortices match standing wavelength rather than shock spacing. Our objective in this study is to verify Panda's observations through computation and to understand the nature of this feedback loop.

We choose fifth order WENO (weighted essentially non oscillatory) scheme for spatial discretization, which is very successful at shock capturing, resolves discontinuities well, and has high accuracy in smooth regions. Temporal discretization is done by third order total variation diminishing (TVD) Runge-Kutta scheme, which does not cause numerical oscillations. Full Navier-Stokes equations are solved with Large Eddy Simulation for turbulence modeling. The domain is chosen similar to the experimental conditions1. Non-reflecting boundary conditions described by Kim and Lee (2000) are used in the far field when the outflow is subsonic, and extrapolation is used for supersonic outflow.

Loh et. al. (2001) studied the same problem using space-time conservation element (CE/SE method). Shen and Tam (1998) used Dispersion Relation Preserving (DRP) scheme and k-epsilon turbulence model to simulate jet screech and compared with former experiments. Both of these models have some disadvantages: The first model uses very complicated unstructured hexagonal grid, which alternates with time 
step. The second uses artificial damping that has to be adjusted in different parts of the domain, such as the nozzle wall corners. On the other hand, our model uses a very simple structural rectangular grid, and no artificial damping is needed.

We select a domain of 12 diameters long in the axial direction and 8 diameters long in the radial direction as in Fig. 4. The flow inside the jet nozzle is a choked flow while outside the nozzle, jet Mach number is $M_{j}=1.19$, and the ambient flow is stationary. These conditions correspond to the experimental setup of Panda (1999) and used by Loh (2001) to model the same problem. The nozzle extends 4 diameters into the domain in order to show backward propagating screech waves. The inflow plane is 3 cells behind the nozzle exit so that instability waves can develop easily at the nozzle exit. The nozzle wall thickness is $D / 4$, as in the experiment. The grid is rectangular, with more points close to nozzle exit in x-direction and nozzle wall in y direction. Polynomials are used to transform from uniform grid to general coordinates, so the metrics are easily obtained analytically. It has been observed that the grid size must be approximately same in each direction (square grid) at the corners of the nozzle wall; otherwise, the solution at these points will diverge.

\subsection{Results and Discussion}

\subsubsection{Case 1: Mach $=1.19$}

We ran our WENO solver for 54000 time steps. We selected the time step such that the maximum CFL number is about 0.5 for convergence. Time averaged solution is recorded as well as the instantaneous result for certain time steps, since the mean flow and shock cell structure is much better visible in the time-averaged results. We also selected our control point for pressure-time history and spectral analysis as $(x, y)=(2,6)$ behind the nozzle, which is also used in (Loh, 2001), for comparison.

Figures 9, 10, 11 show time averaged values of density, pressure, and numerical Schlieren plot, which is a contour plot of density gradient in x-direction, 
matching the experimental setup of Panda (1999). These agree well with Panda's experiment and Loh's computation. Figure 12 is the experimental Schlieren photograph. It can be seen in Figure 9 that the shear layer starts from the nozzle exit and spreads out downstream as the instability wave interacts with shock cells and increases its amplitude. The First three shock cells are clearly visible in all plots. Fourth cell and beyond are affected by the instability vortices and begin to weaken, which is also the case in the experiment.

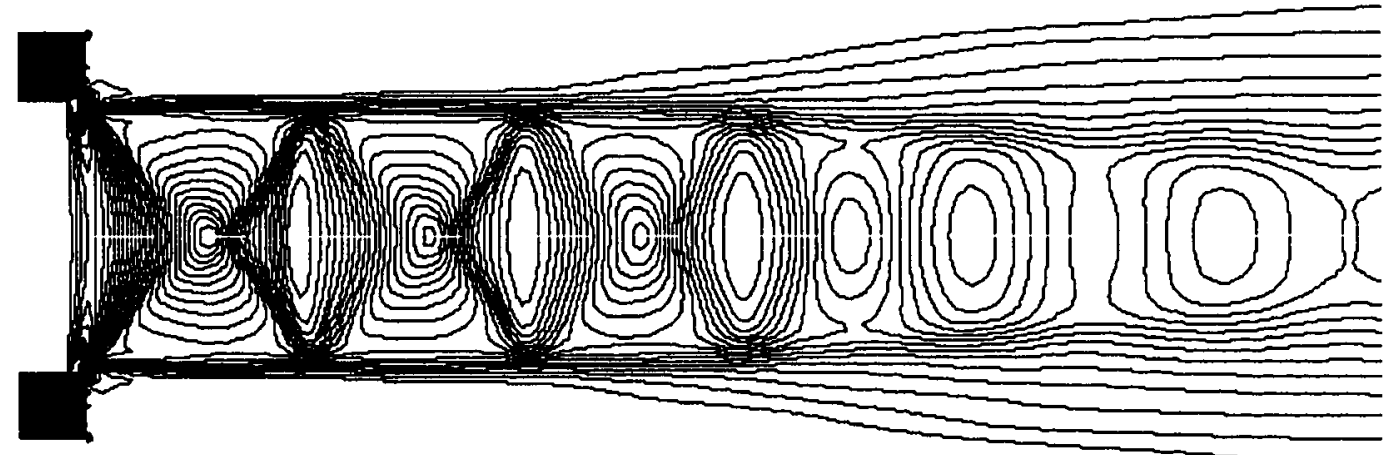

Figure 9: Time averaged density contours after 40000 steps.

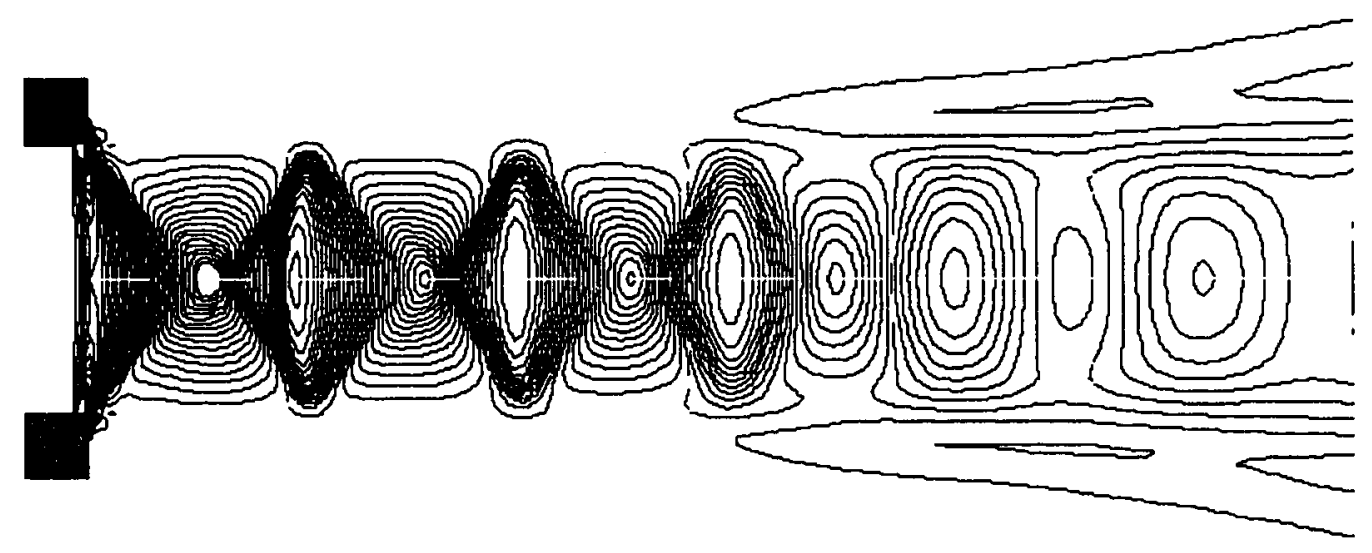

Figure 10: Time averaged pressure contours after 40000 steps. 


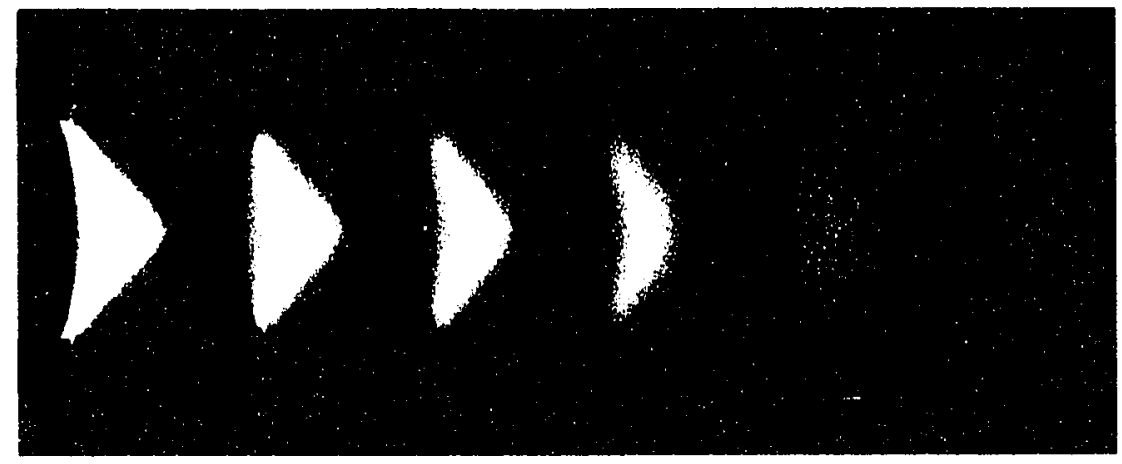

Figure 11: Numerical Schlieren

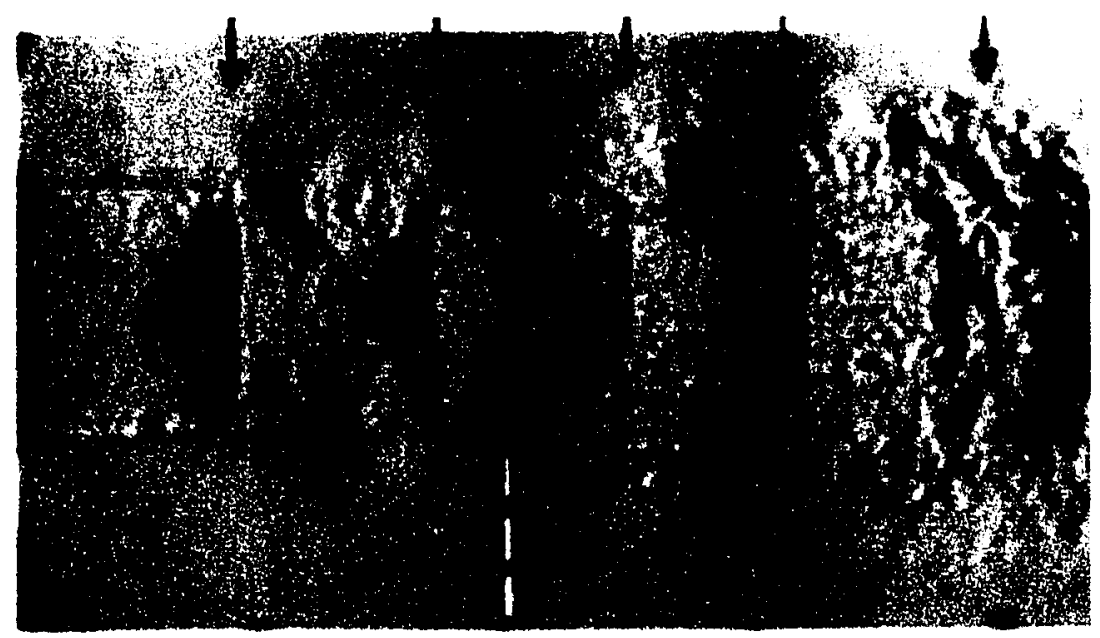

Figure 12: Experimental Schlieren

Figure 13 shows a contour plot of instantaneous pressure. Contour values are adjusted such that the screech waves can be seen clearly. This plot is in agreement with Loh's calculations. Looking at the distance between thick contour lines away from the nozzle, the wavelength of screech waves can be roughly estimated as $1.5 \mathrm{D}$, which is estimated by Loh to be 1.6 D. 


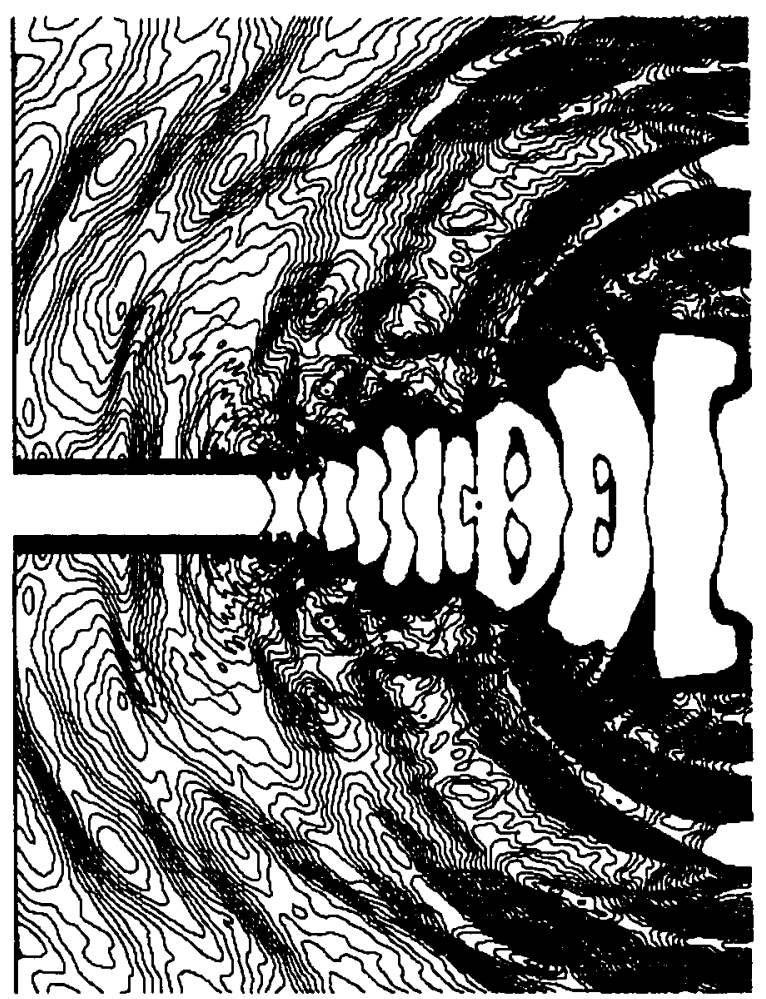

Figure 13: Instantaneous pressure contours, screech waves

Figure 14 shows the time history of pressure at the control point $(x=2, y=6)$. Figure 15 shows the sound pressure level at that point same point vs. frequency in Hertz. Pressure history is post processed by taking the FFT transform to obtain SPL. The highest amplitude occurs at $8470 \mathrm{~Hz}$, which corresponds to screech frequency.

An animation of density contours clearly shows the formation of instability vortices and their interaction with the shock waves. Twelve selected frames of this animation are shown in Figure 16a-161. Each frame is 500 time steps ahead of the previous one. Especially the top part of the first compression wave moves back and forth, as each vortex passes by in the flow direction and the next one comes on top of it. Growth of vortices in the downstream direction and their leaving the computational domain on the right side is also visible. Figures do not include the whole domain which goes from the origin up to $\mathrm{x}=12$ and $\mathrm{y}=8$, however, whole domain is included in the video animation. Propagation of sound waves from the first three cells 
towards outside from the jet core is clearly observed. The generation of sound waves through interaction with the hydrodynamic waves is similar to Panda's experimental observations. It is also remarkable that the cells beyond the first one appear and disappear during the cycle, whereas they look firm and clear in the time-averaged plots.

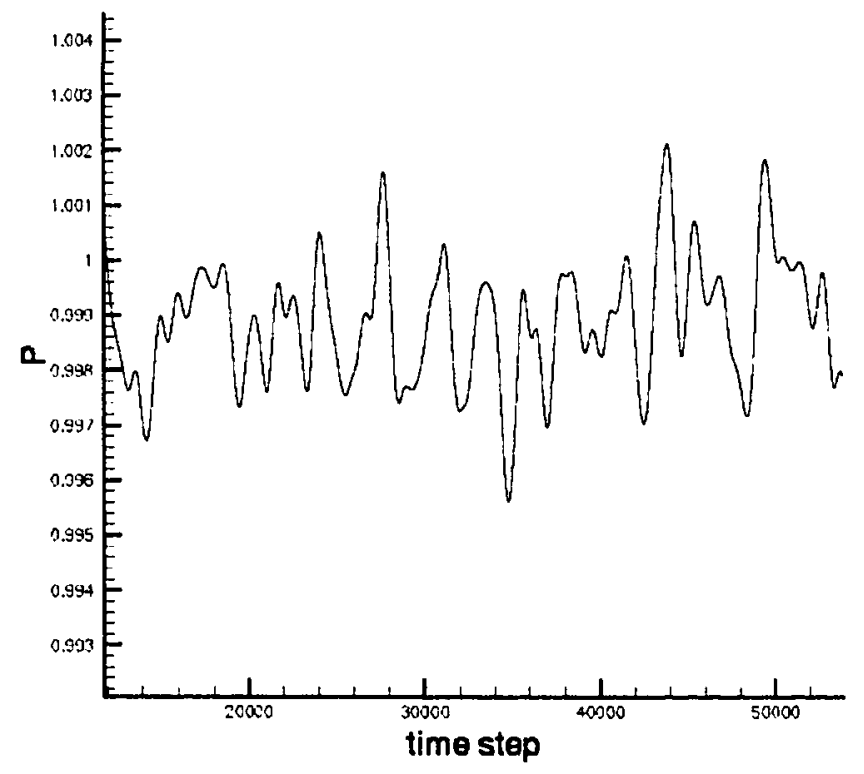

Figure 14 Pressure history at $(2.0,6.0)$ 


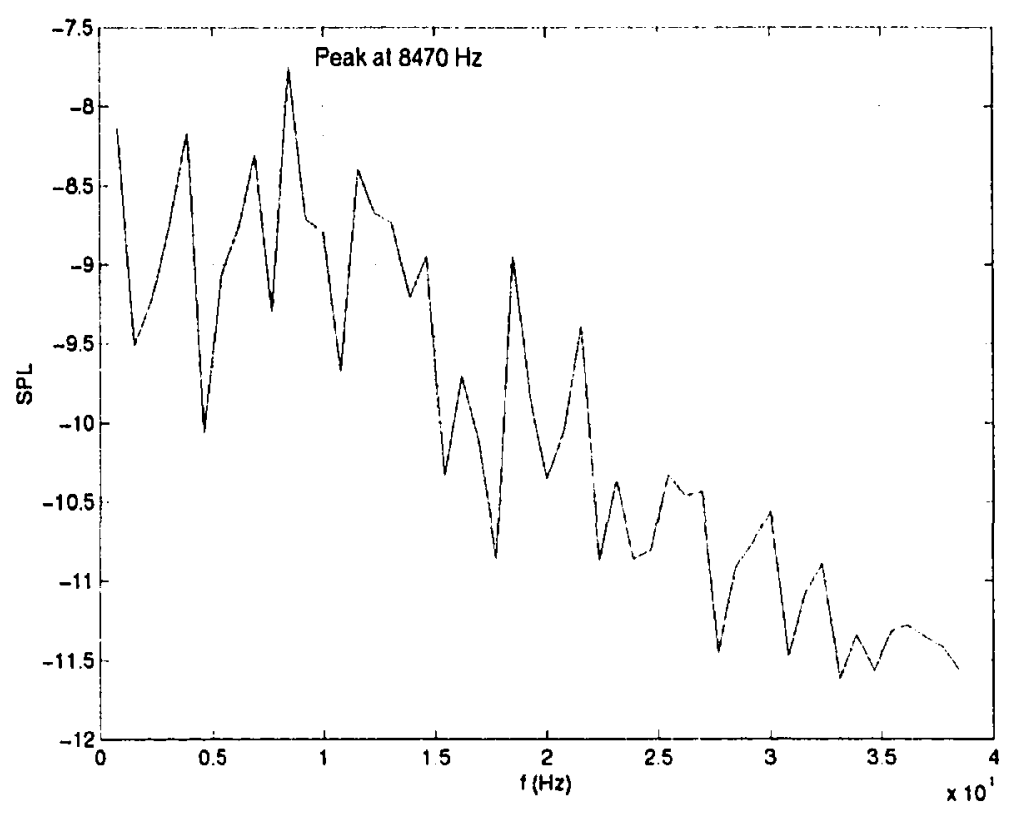

Figure $15 \mathrm{SPL}$ at point $(2.0,6.0)$

The shock cell structure and the computational screech frequency of $8470 \mathrm{~Hz}$. is in very good agreement with the experimental value of $8400 \mathrm{~Hz}$. These results were obtained without introducing any periodic forcing function. The inflow boundary conditions at the nozzle exit and the nozzle lip create instability vortices and the interaction of these with the shock cells generates screech waves seen at the far field. Therefore, we can conclude that WENO model is efficient and accurate for computation of shock-induced noise. 


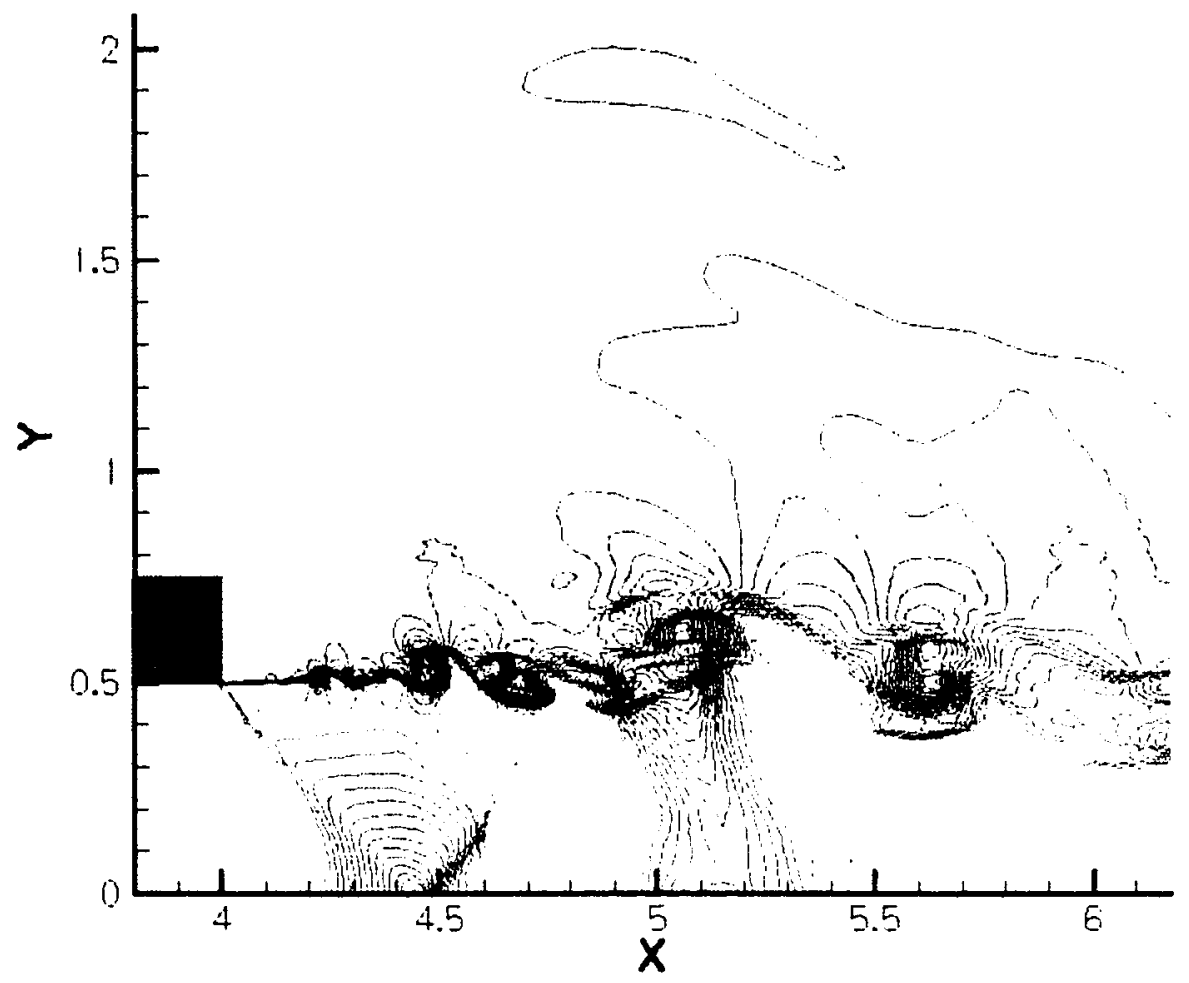

Figure 16a: Initial frame of animation, density contours.

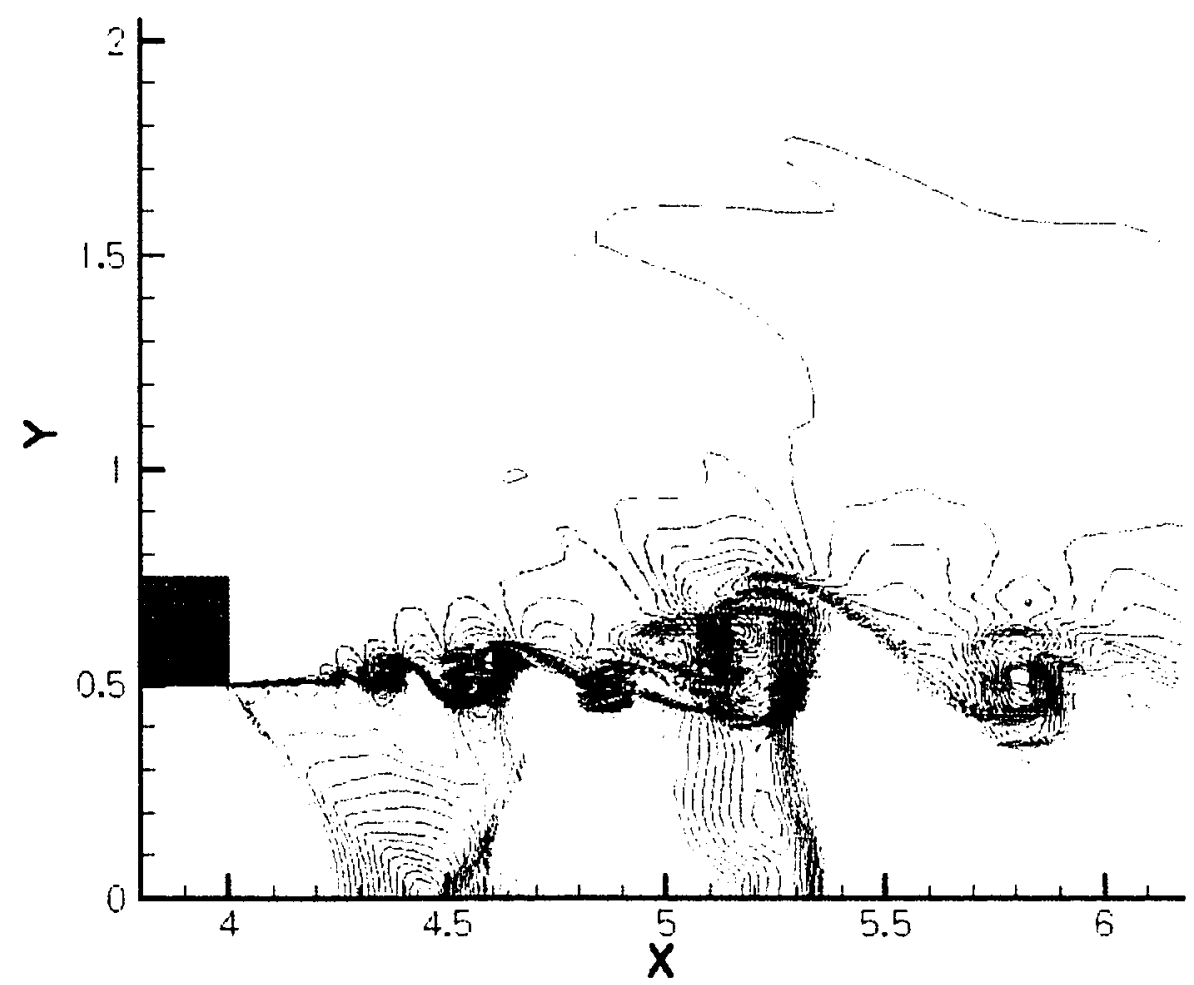

Figure 16b: After 500 time steps. 


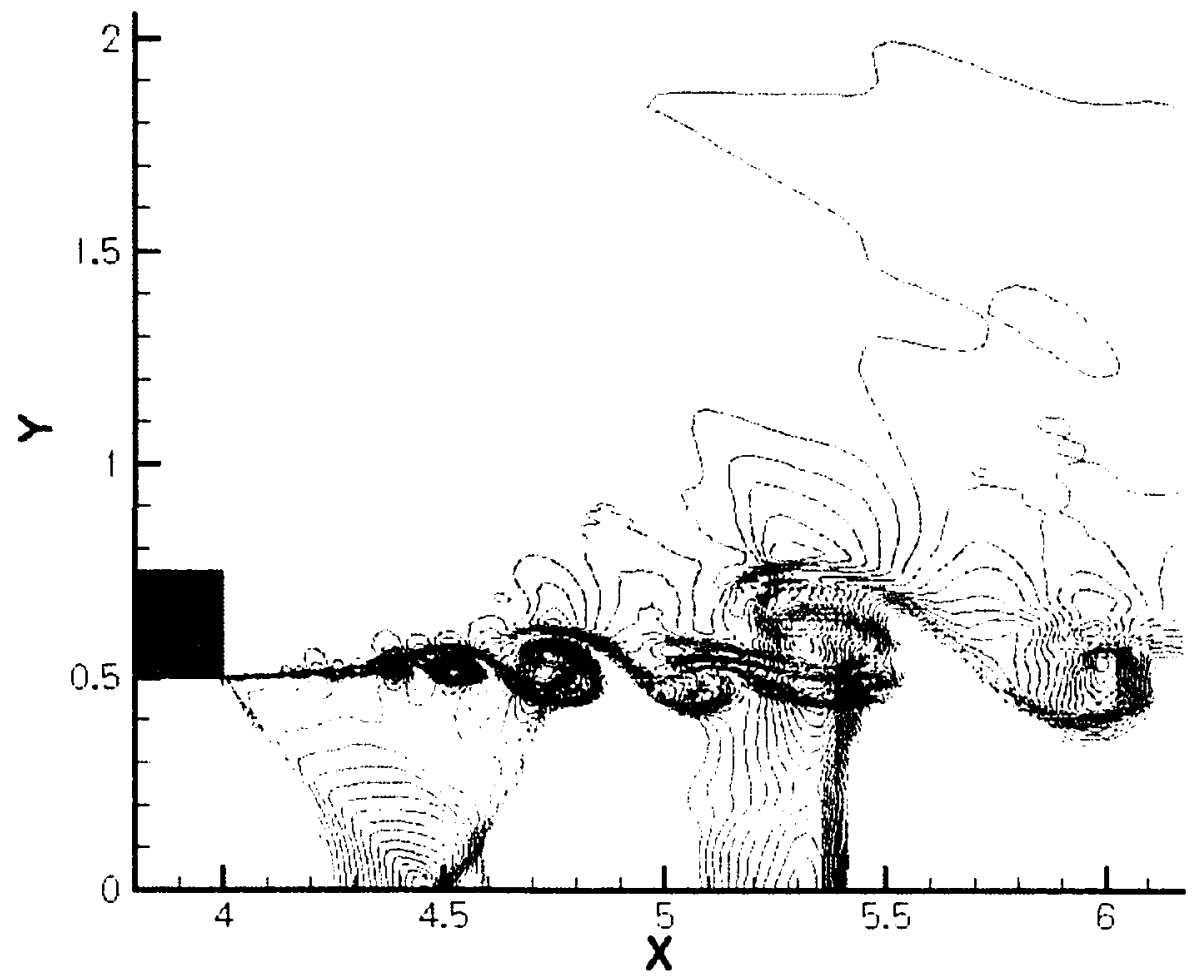

Figure 16c: After 1000 time steps.

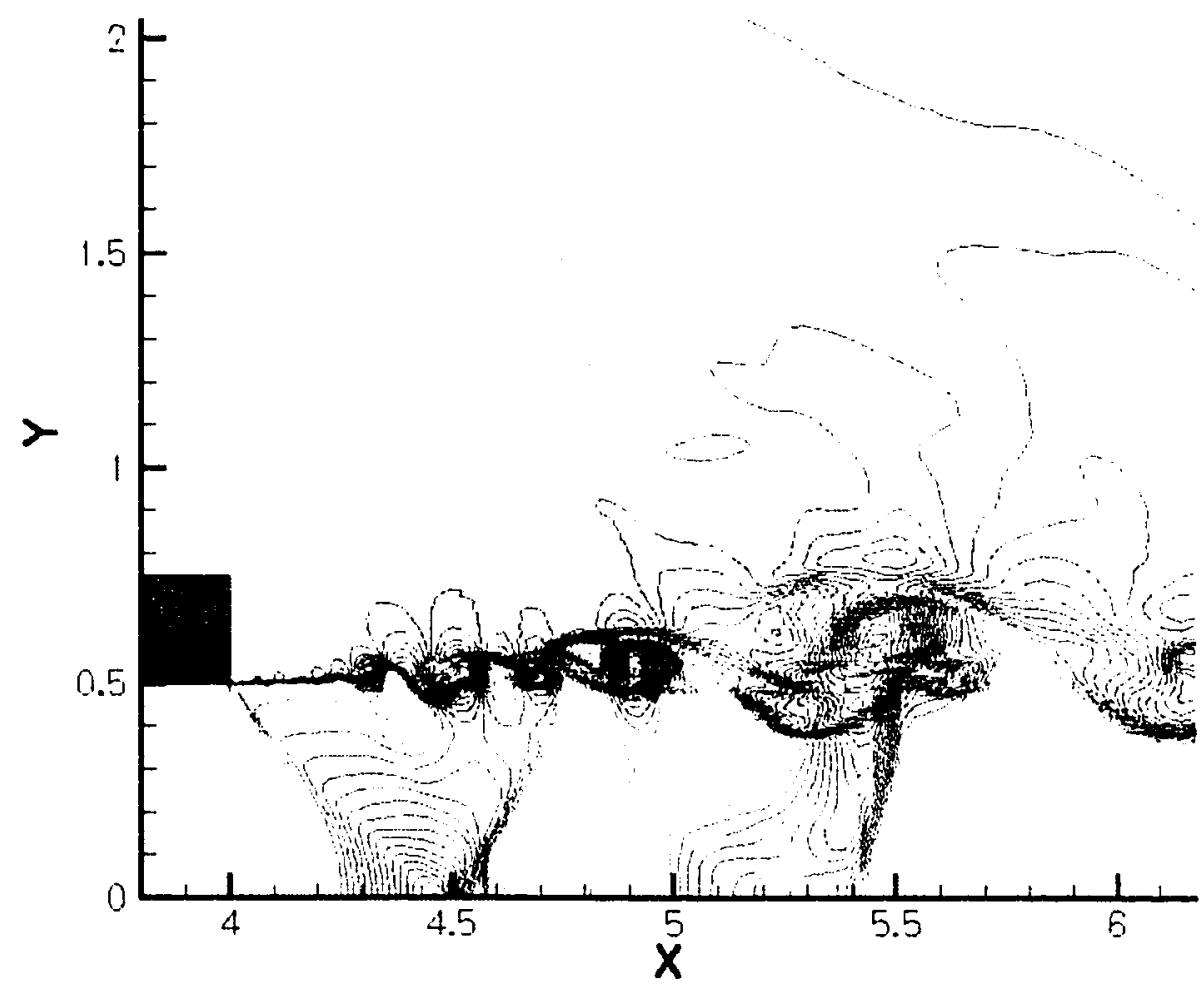

Figure 16d: After 1500 time steps. 


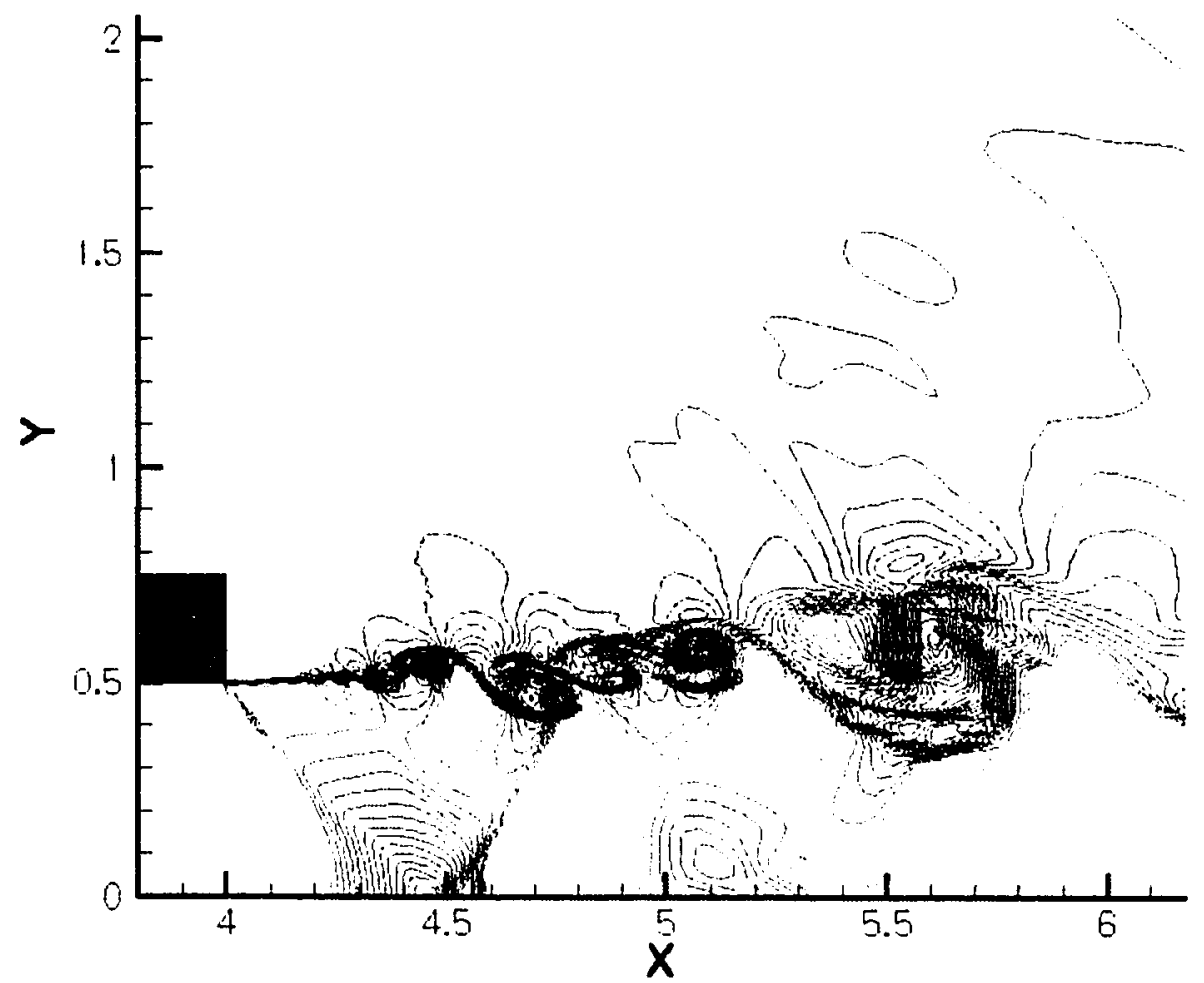

Figure 16e: After 2000 time steps.

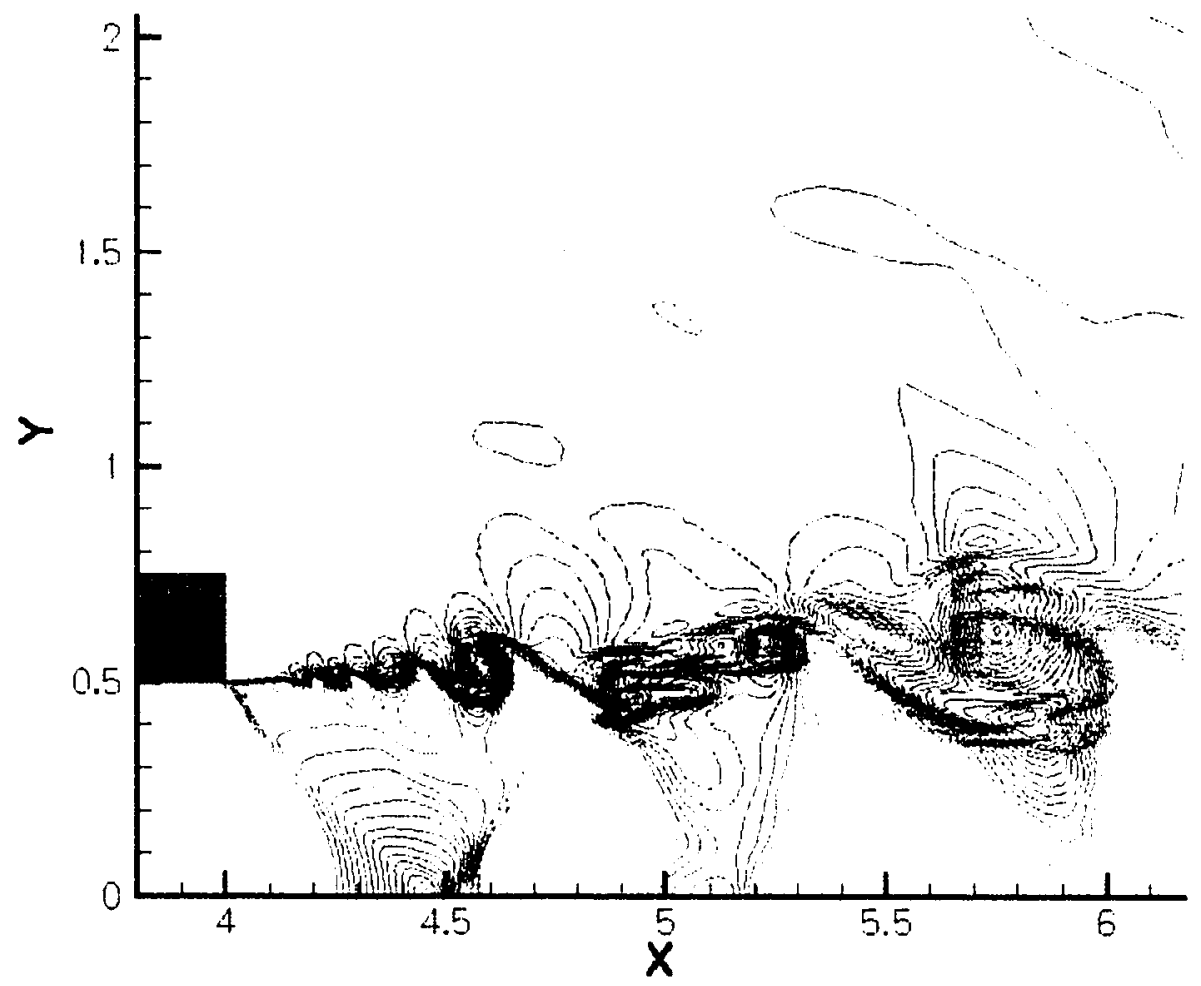

Figure 16f: After 2500 time steps. 


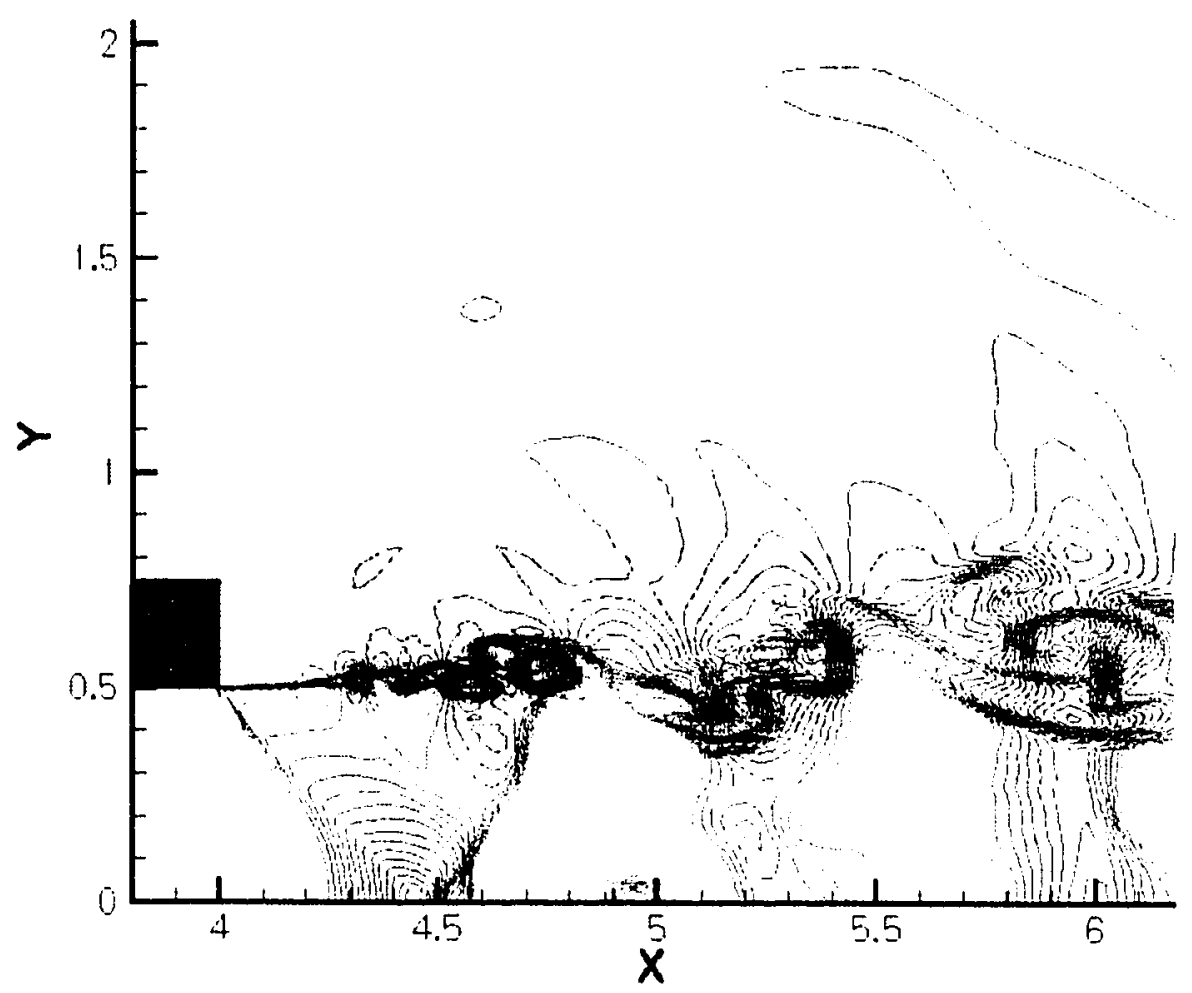

Figure 16g: After 3000 time steps.

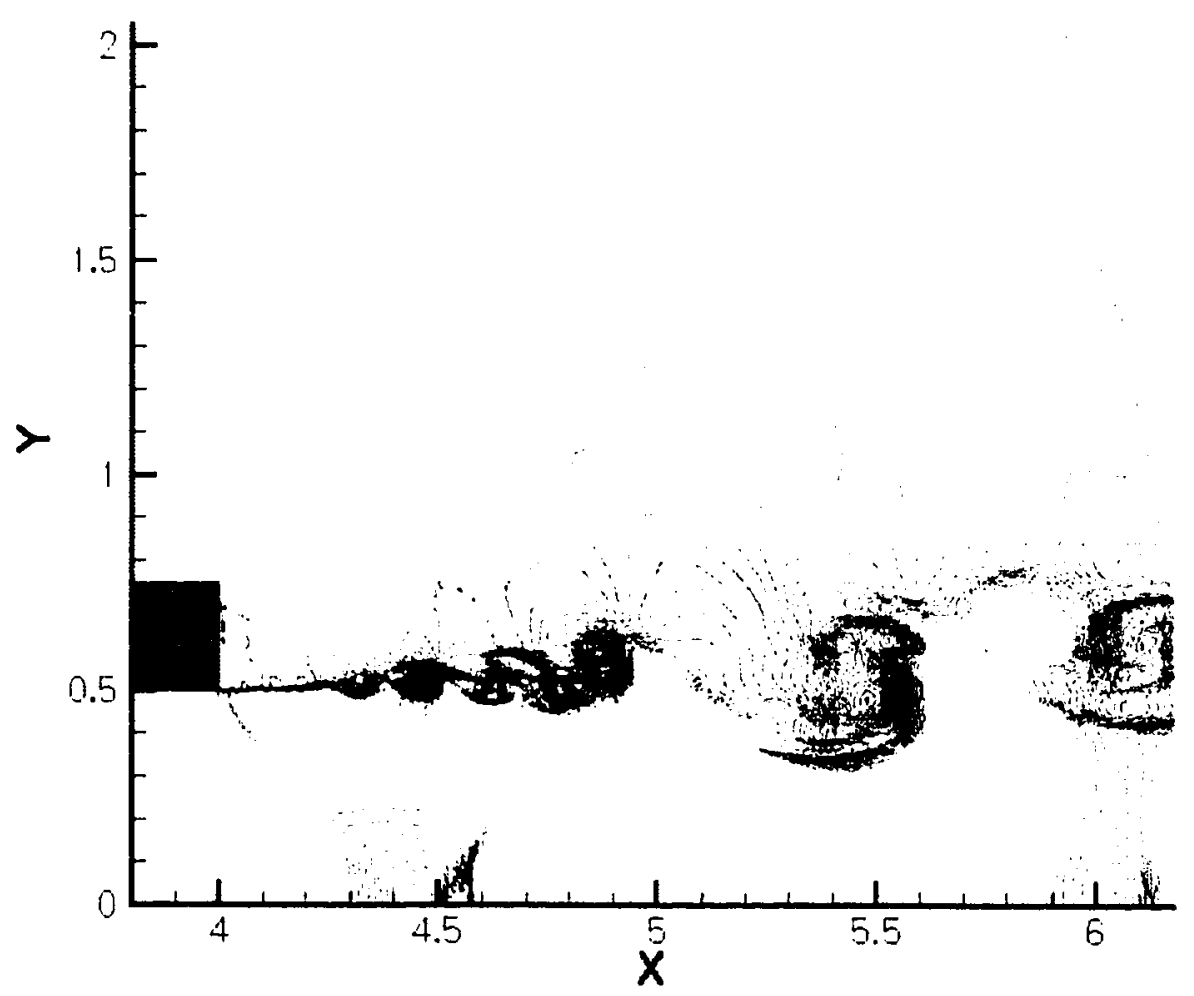

Figure 16h: After 3500 time steps. 


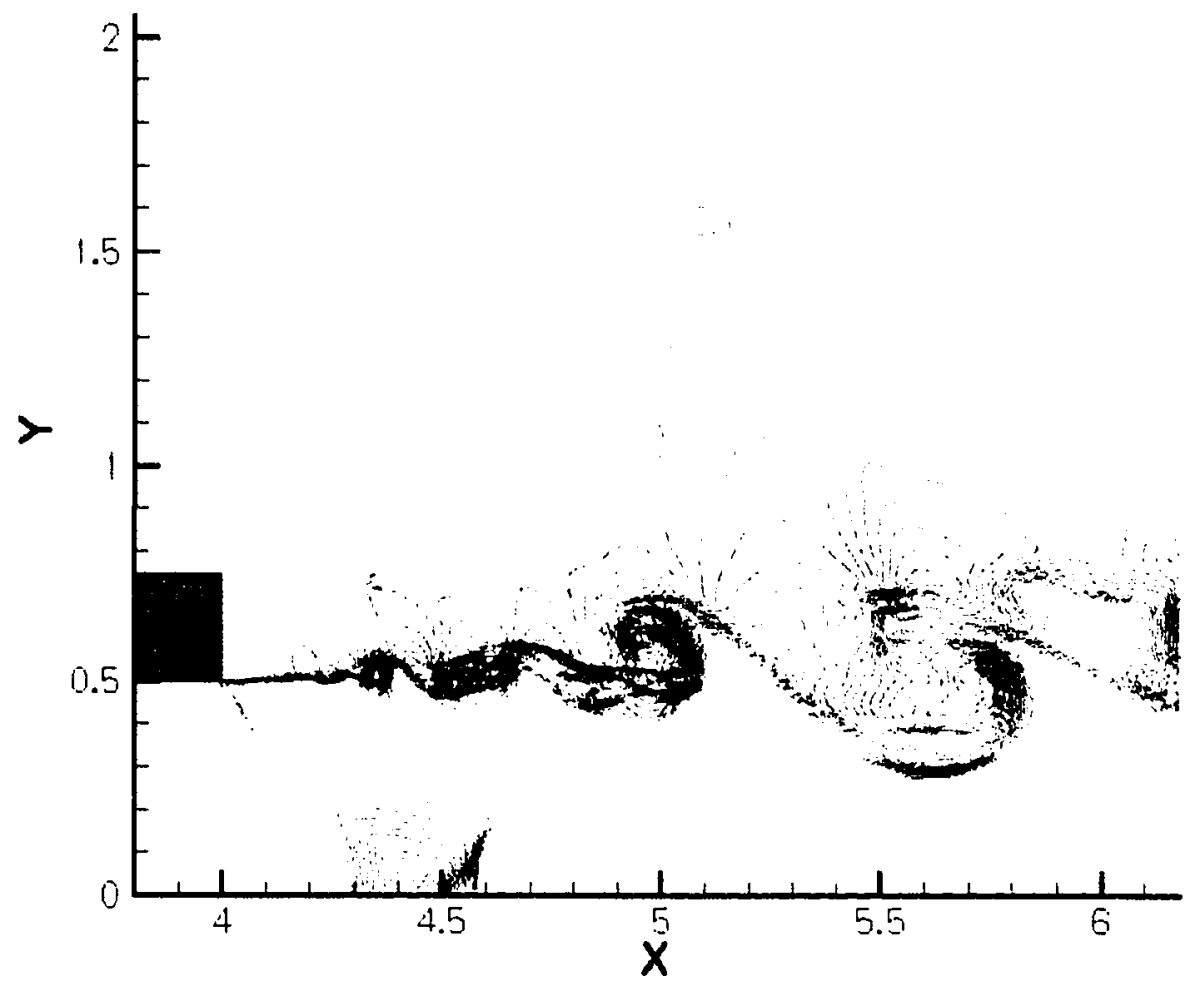

Figure 16i: After 4000 time steps.

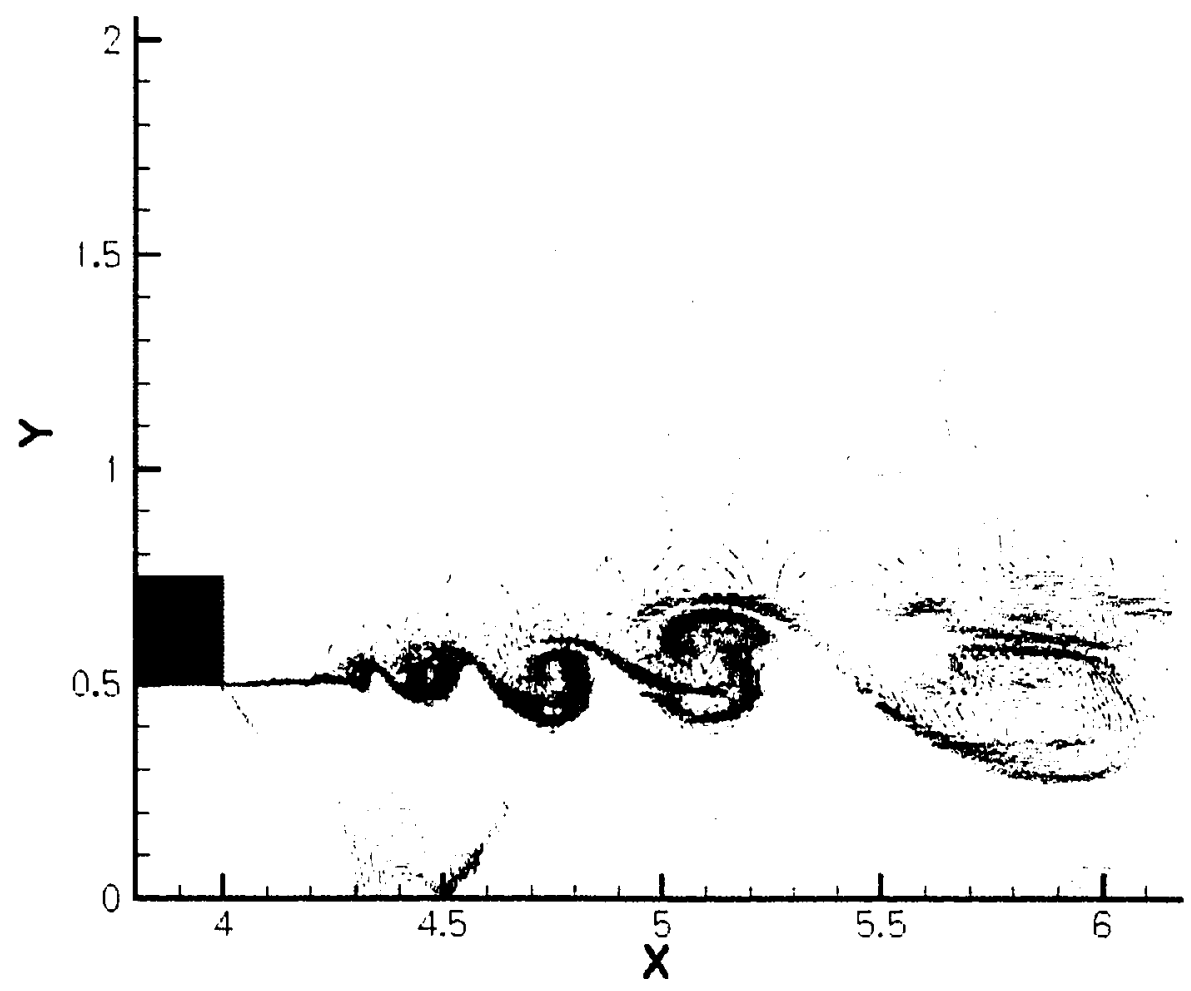

Figure 16j: After 4500 time steps. 


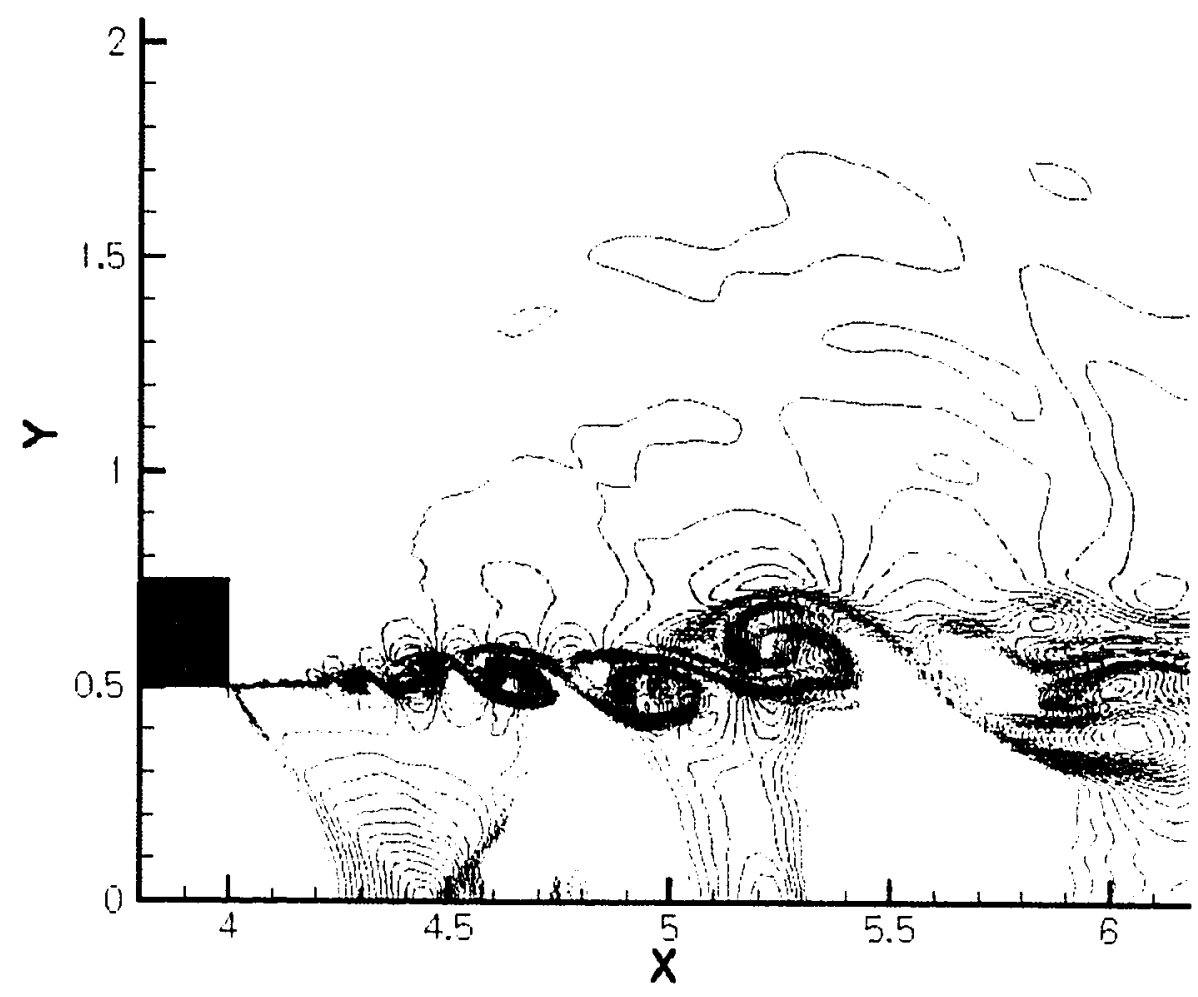

Figure 16k: After 5000 time steps.

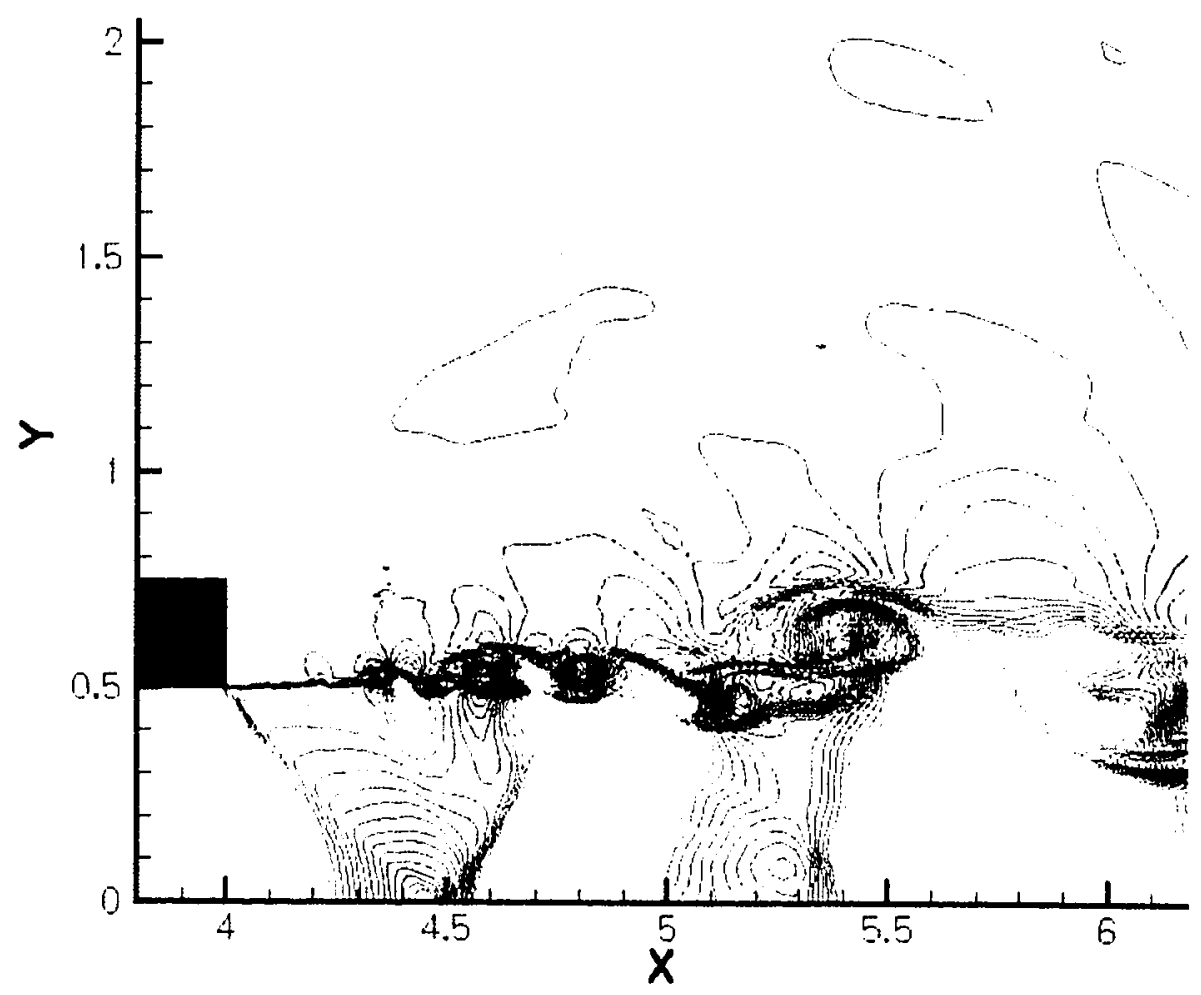

Figure 161: After 5500 time steps. 


\subsubsection{Case 2: $\operatorname{Mach}=1.43$}

At this Mach number, experiments have shown helical mode instability. Even when the case is simulated by assuming axisymmetric flow, the same WENO code computes the mean flow pattern and estimate the screech frequency in very well agreement with experimental results.

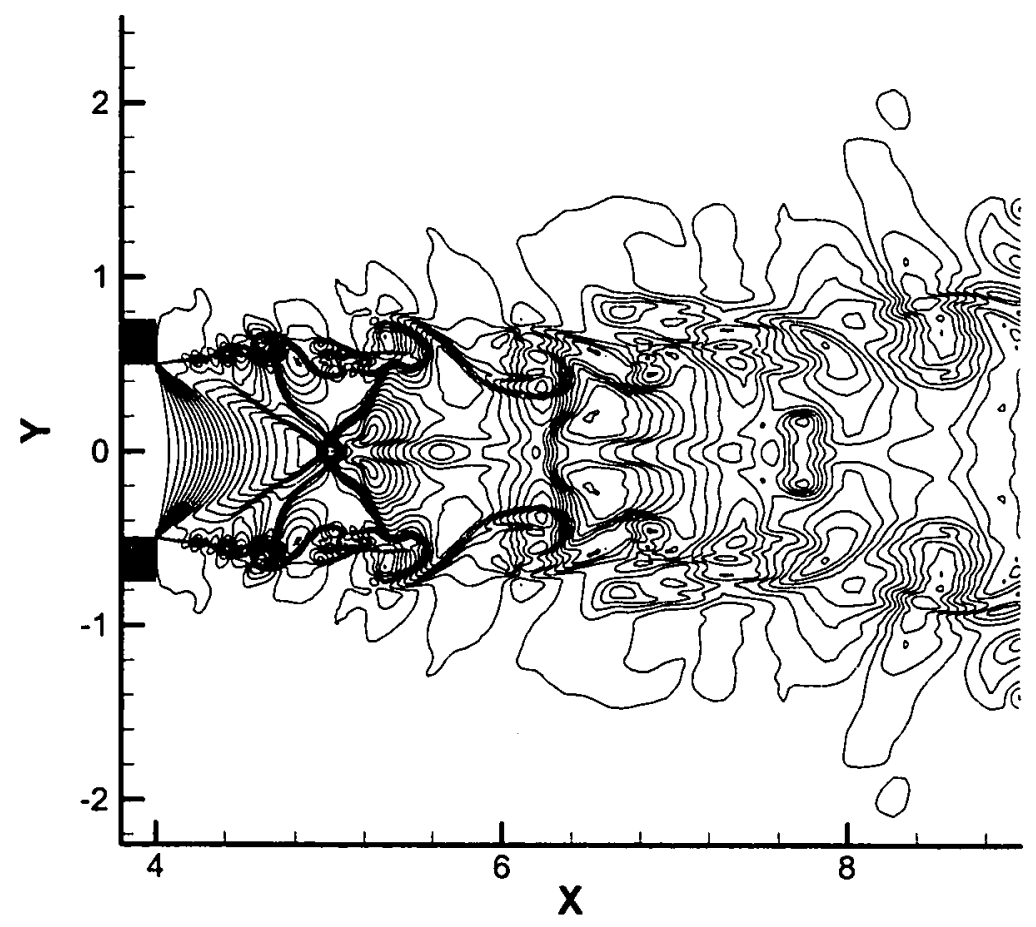

Figure 17 Instantaneous density contours after 150,000 time steps. 


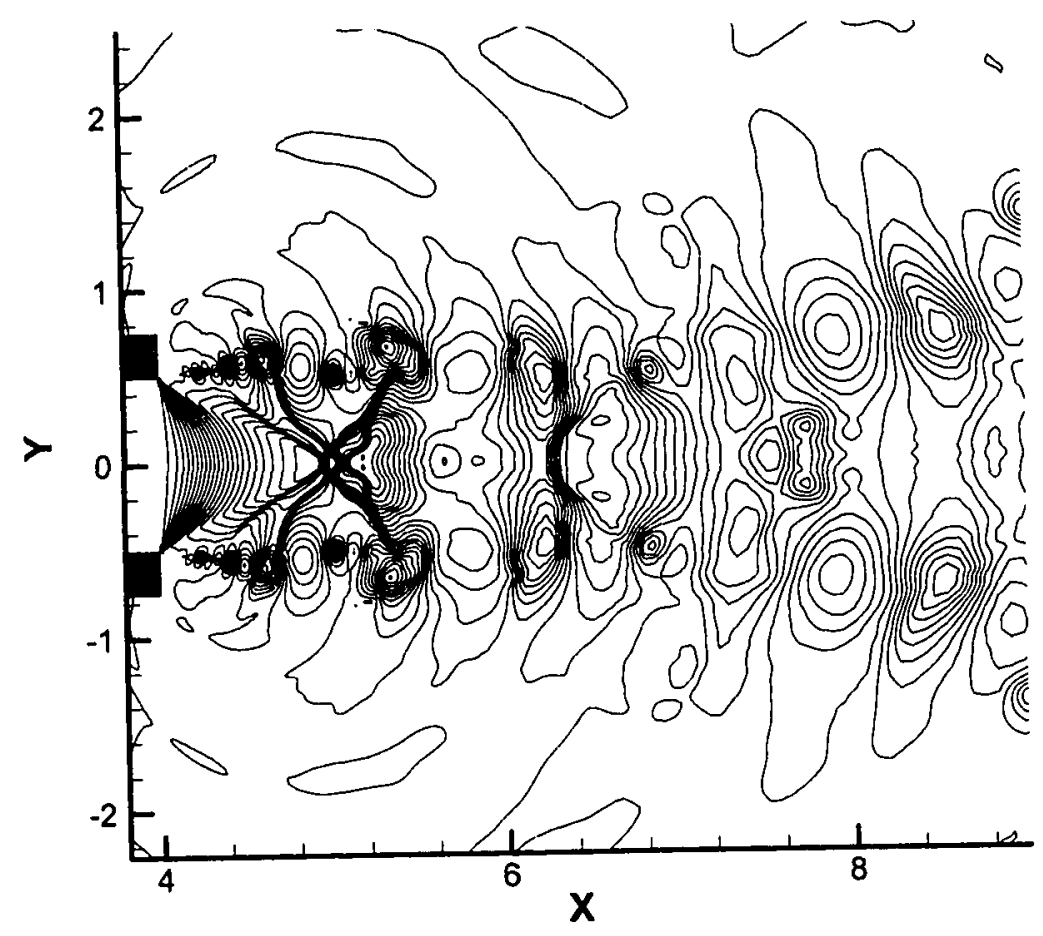

Figure 18 Instantaneous pressure contours after 150,000 time steps.

Figures 17 and 18 show the instantaneous density and pressure contours for Mach number 1.43. Comparing with Figures 9 and 10 for Mach 1.19, it can be observed that the shock cells have become longer and the shear layer is slightly expanded. The shock cell structure is not as clear as in the lower speed case. Same observations can be made for Figures 19 and 20, which show time averaged density and pressure contours. The first shock is very clear in these time-averaged graphs, but the second and later ones are getting weaker. The numerical Schlieren shown in Fig. 21 gives a better view of the third shock cell. Comparing with the experimental Schlieren in Figure 22 validates the location of shocks. It should be noted that the scale in Fig. 21 starts at $x=4$, but it starts from zero in Fig. 22. In both figures, the first expansion wave ends about $0.9 \mathrm{D}$ from the nozzle, and the first cell ends at 1.25 D. The end of the second expansion is $2 \mathrm{D}$ from the nozzle in both figures. 


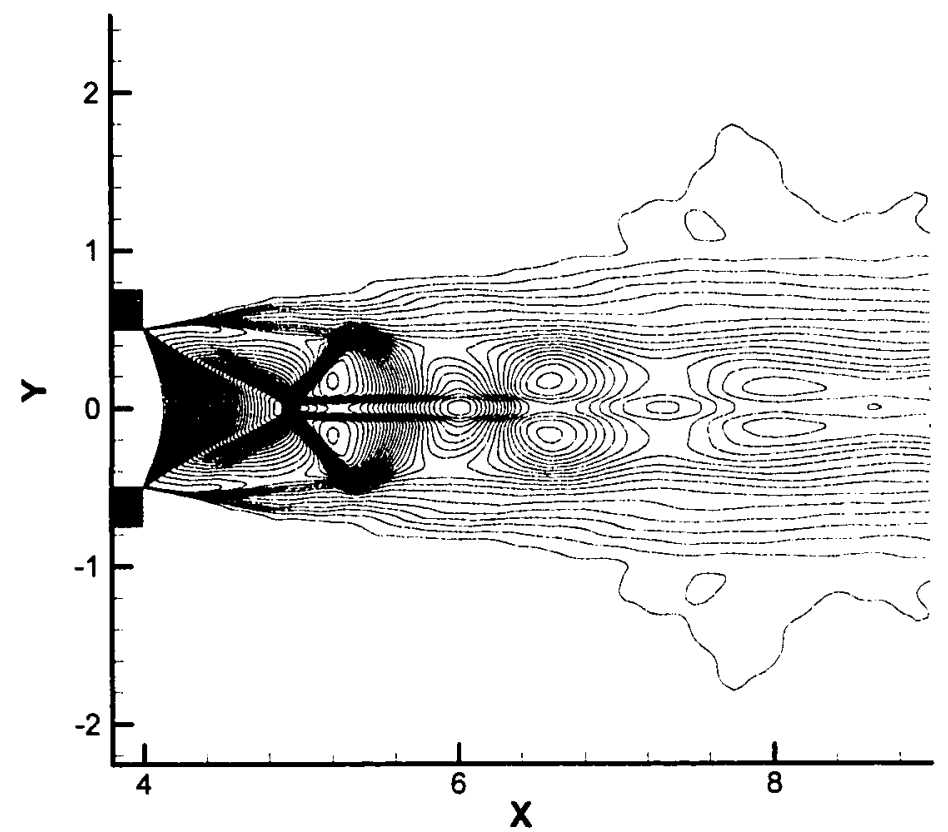

Figure 19 Time averaged density contours after 150,000 time steps.

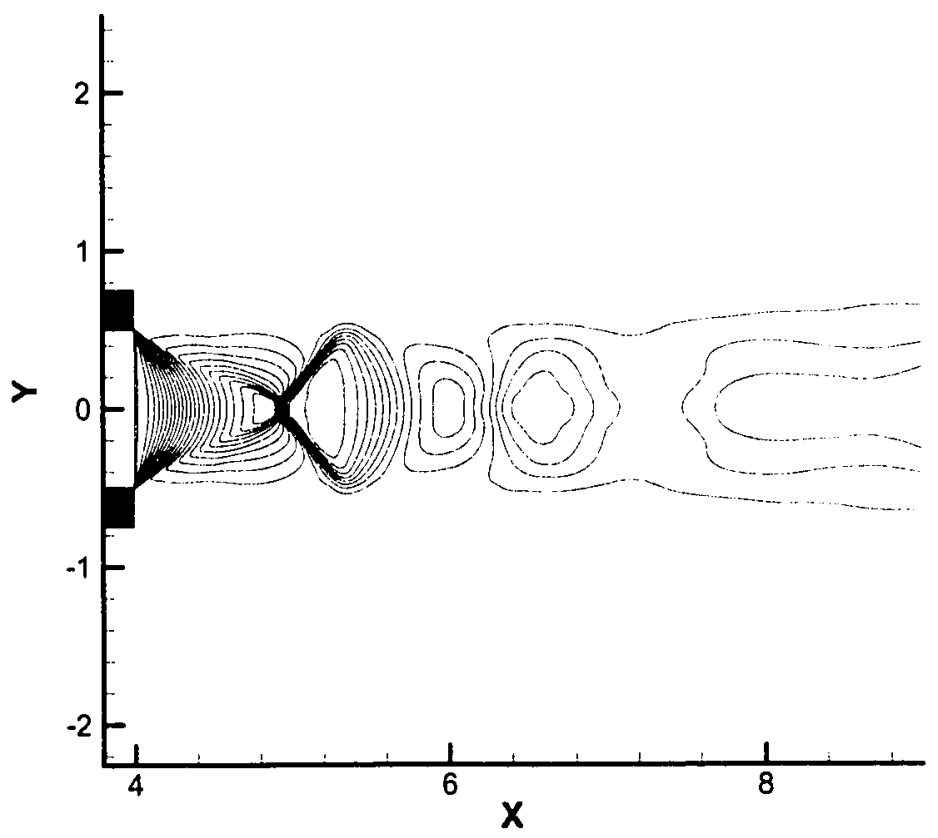

Figure 20 Time averaged pressure contours after 150,000 steps. 


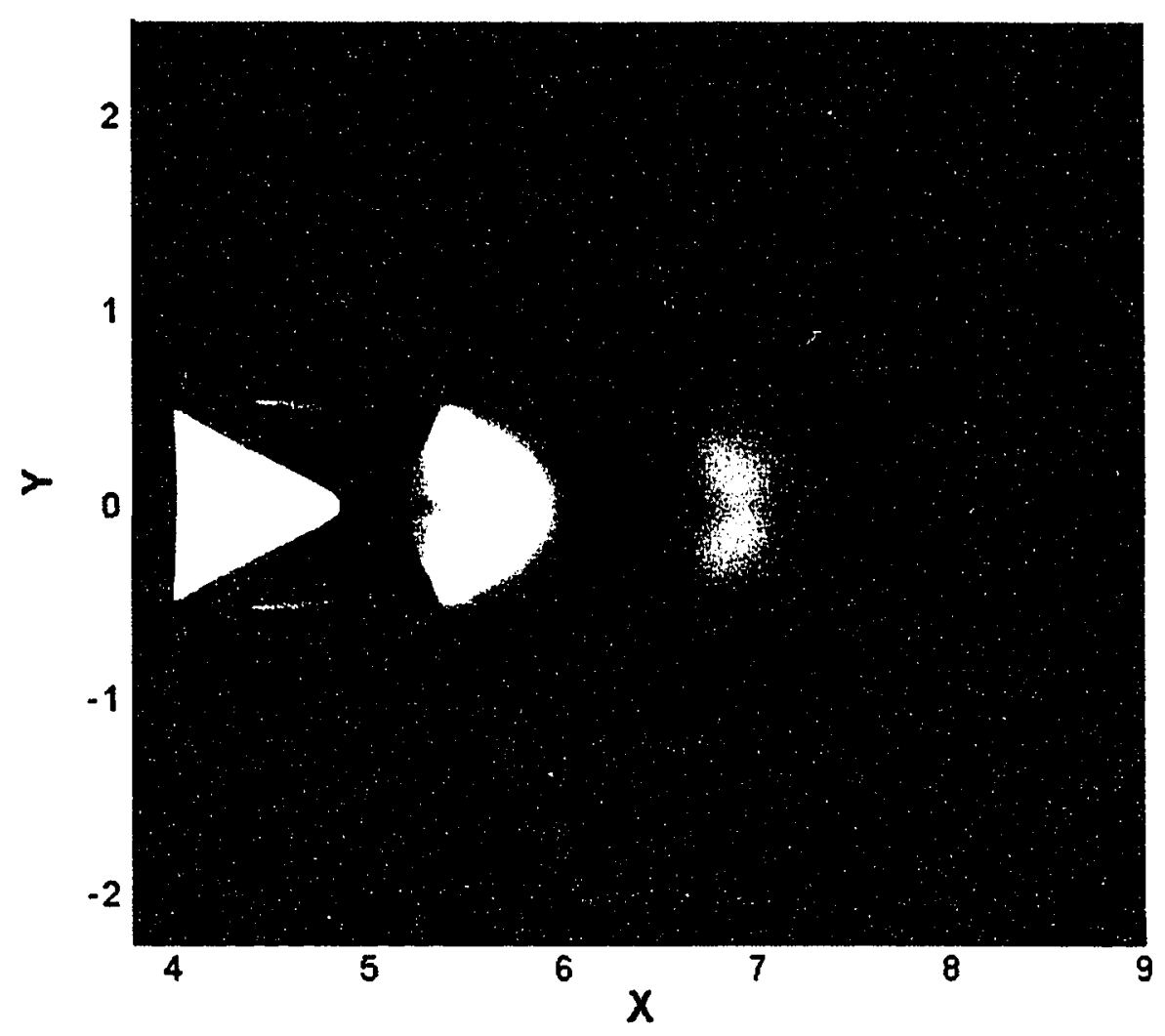

Figure 21 Numerical Schlieren

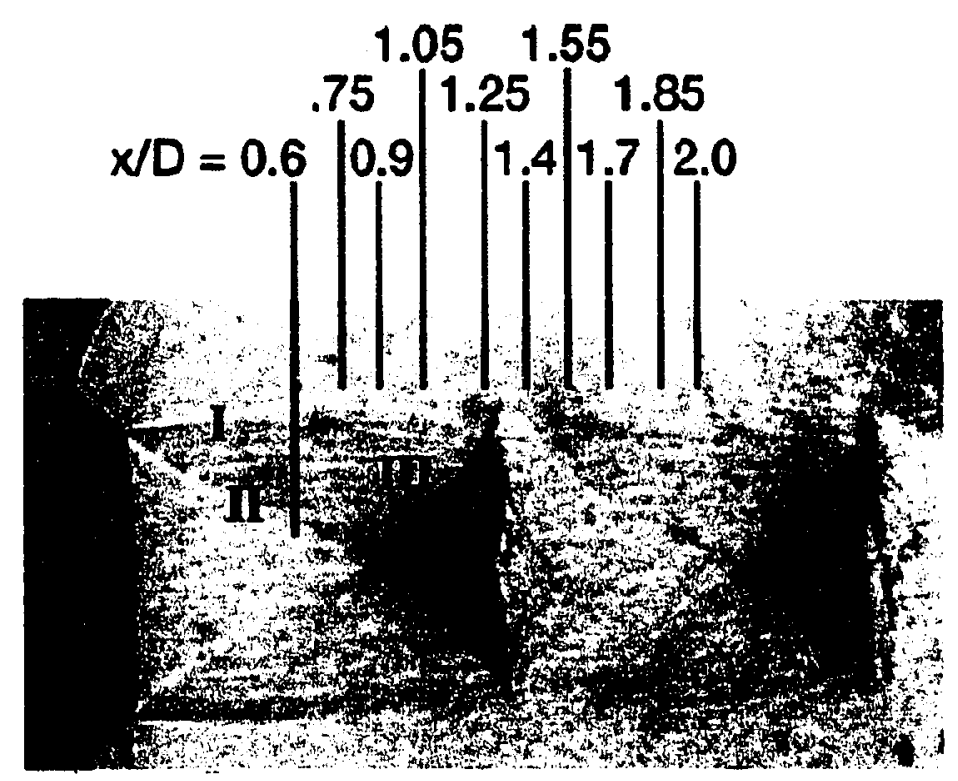

Figure 22: Experimental Schlieren for $M=1.43$ 
In Figure 23, for a selected constant axial location, $x=0.6$ from nozzle, density versus $y$ (radial distance) is plotted for both experimental and numerical results. Experiment data is from the article (Panda, Seasholtz, 1999) and is available on the internet (See EPAPS in References). This is a good agreement for both values and locations of start of increase and decrease. Going back to Figure 22, we see that this is where the expansion takes place at the center part of the jet and the upper part is in the compression zone. Our results for Mach 1.43 using axisymmetric model are very good, but we want to see what happens in three dimensions.

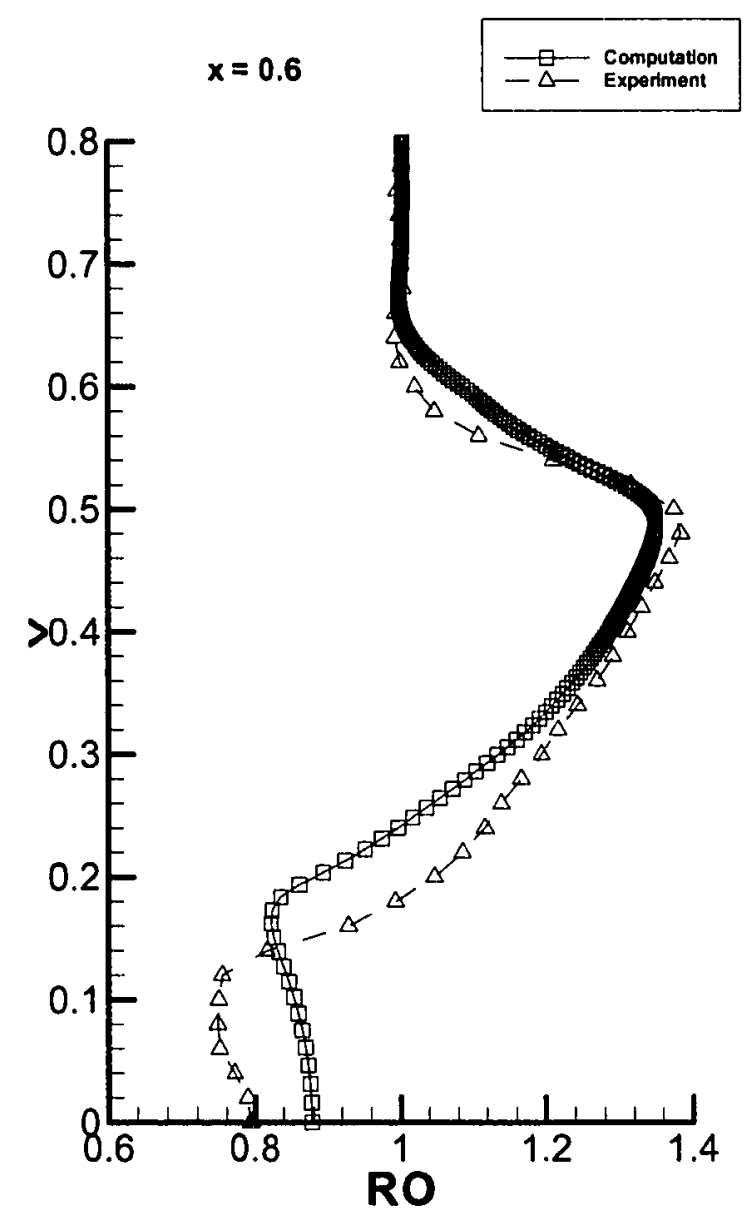

Figure 23 Comparison with experimental results. 


\section{CHAPTER 6 \\ THREE-DIMENSIONAL SIMULATION RESULTS}

\subsection{Introduction}

Our results for Mach number 1.43 in two dimensional axisymmetric configuration have been satisfactory for general flow quantities, like shock cell pattern and screech frequency prediction. However, experimental results for this case show three-dimensional effects like helical modes. To understand the threedimensional effects at higher Mach numbers, the simulation is extended to threedimensions. First, a higher Mach number of 1.80 , which is also a case in experiments by Panda (1999), will be solved to verify that our code is capable of resolving shock patterns at these high Mach numbers. This case is also interesting, as it shows how the shock cell pattern varies and barrel shocks occur. Then, the previous case of Mach 1.43 will be analyzed again, this time in three-dimensions. At this Mach number, experimental, two- and three-dimensional results will be compared, and what happens in the angular direction will be investigated. Due to computational restrictions, the domain has to be smaller in axial and radial directions for three-dimensional computations.

To go from two to three dimensions, the grid is revolved 180 degrees around the $\mathrm{x}$-axis and divided into 16 equal slices. Governing equations are modified to include angular and source terms, as shown in chapter 4.

\subsection{Results and Discussion}

We ran our WENO solver for 12000 time steps for the 3-D case, 150000 steps for axisymmetric case and 32000 steps for another case of Mach number 1.80. The last case is for comparison with experimental results and to verify the shock capturing capability of our code. In all cases, we selected the time step such that the maximum CFL number is about 0.5 for convergence. Time averaged solution is recorded as well 
as the instantaneous result for certain time steps, since the mean flow and shock cell structure is much better visible in the time-averaged results.

Figures 24 and 25 show time averaged and instantaneous values of density for Mach 1.8 jet. In this high-speed flow, the shear layer is elevated and the transition from expansion to compression occurs in the form of a barrel shock. The shape of shock cell structures and the location of barrel shock in our calculation agree well with experimental results, since the barrel is $1.5 \mathrm{D}$ away from the nozzle in both. As in the axisymmetric case and experiments, shock strength decreases in the downstream direction, so the first shock after the nozzle is the strongest one.

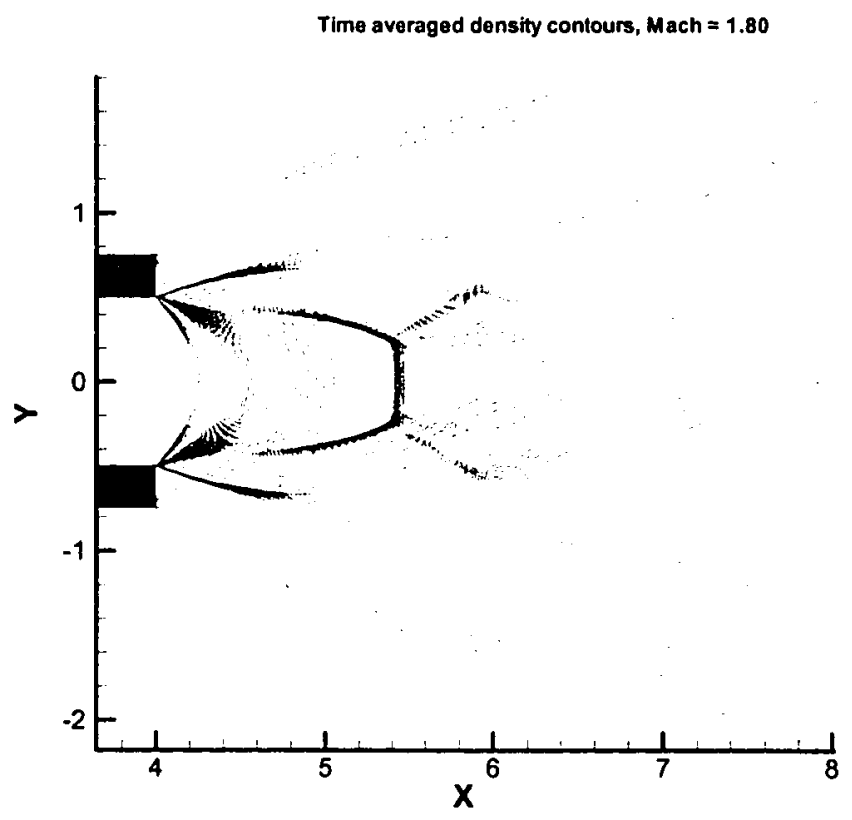

Figure 24: Time averaged density contours after 30000 steps, Mach $=1.80$ 


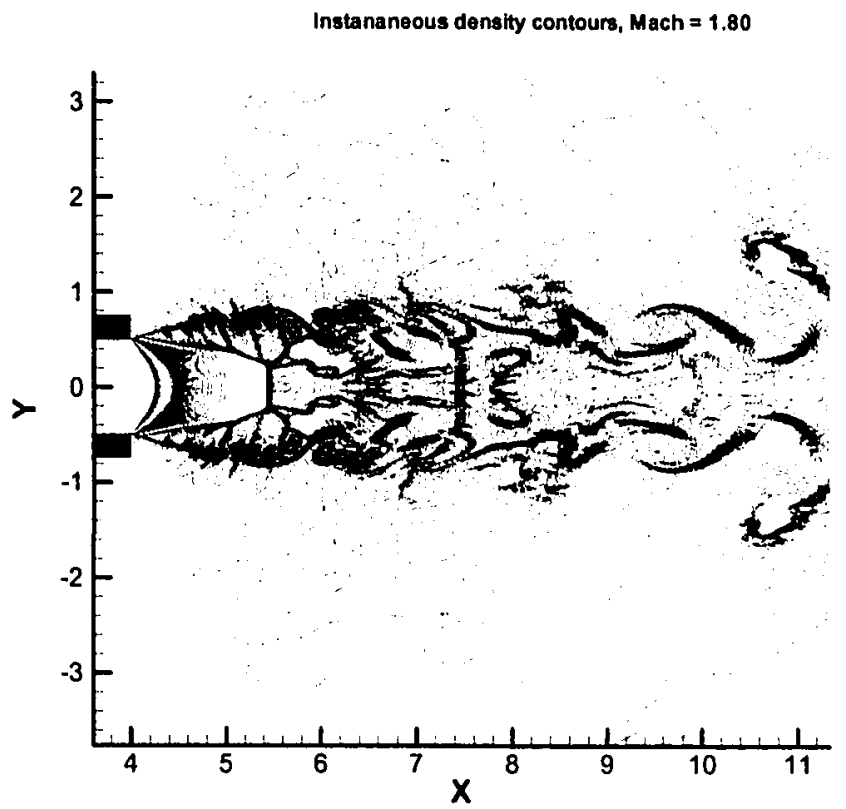

Figure 25: Instantaneous density contours after 32000 steps, Mach $=1.80$

Figures 26 and 27 are two-dimensional time averaged and instantaneous density contours for Mach number 1.43. This time, the first expansion ends at $0.9 \mathrm{D}$ and the first cell goes up to 1.25 D from nozzle. The cell shape is in agreement with the experiments. The numerical Schlieren in Figure 28 is obtained by plotting contours of $x$-derivative of density, therefore it is comparable to the experimental Schlieren plots of Panda (1999). The shock cell pattern is seen clearer in Fig. 28. 


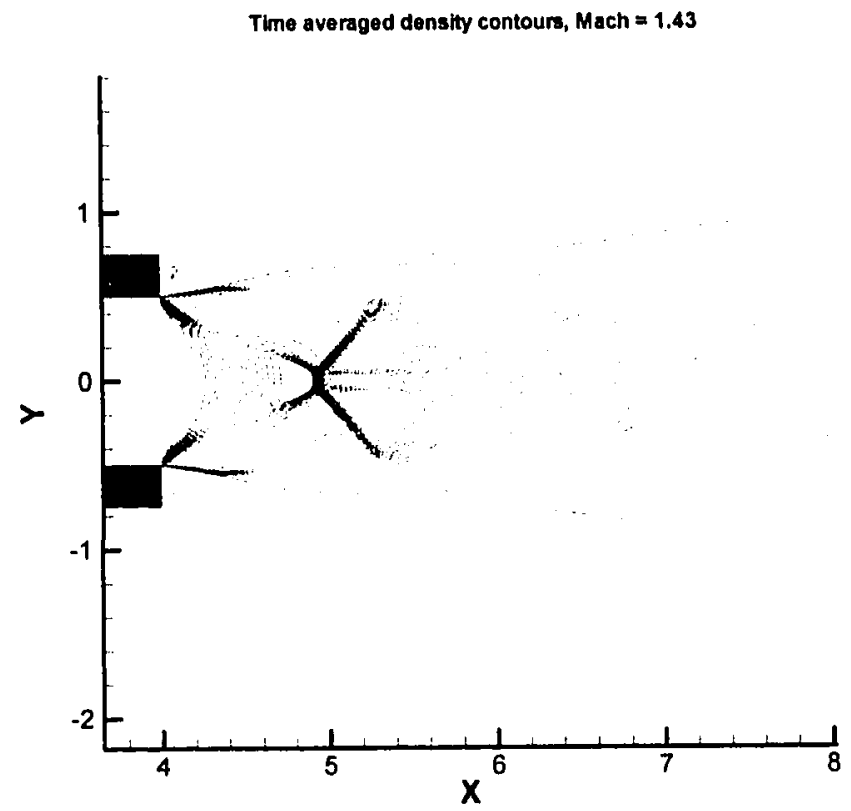

Figure 26: Time averaged density contours after 150000 steps, Mach $=1.43$

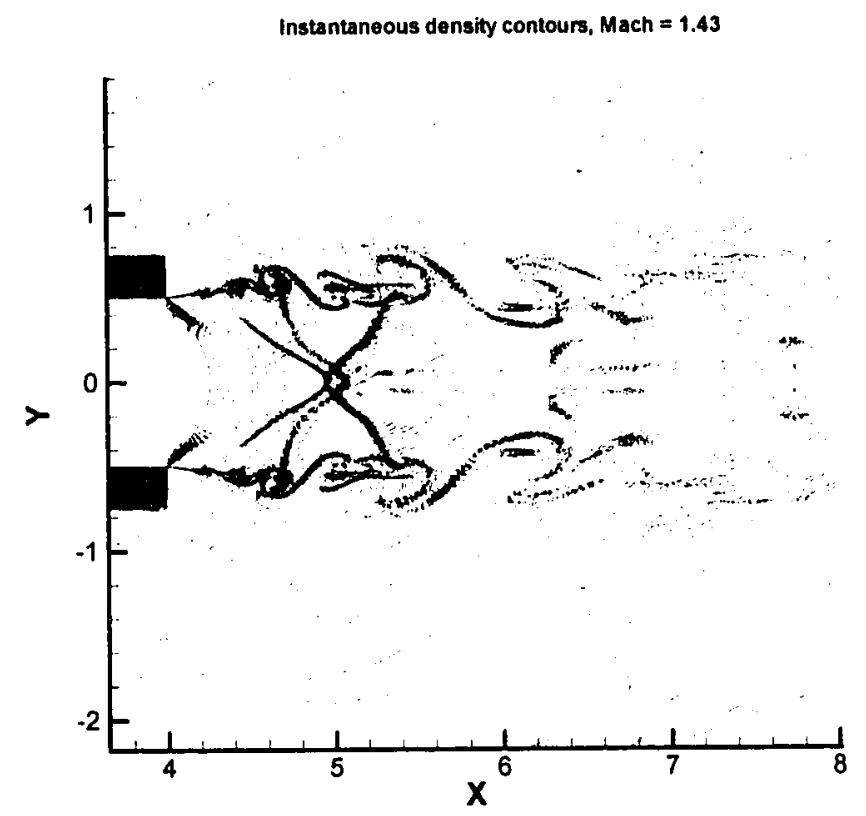

Figure 27: Instantaneous density contours 150000 steps, Mach $=1.43$ 


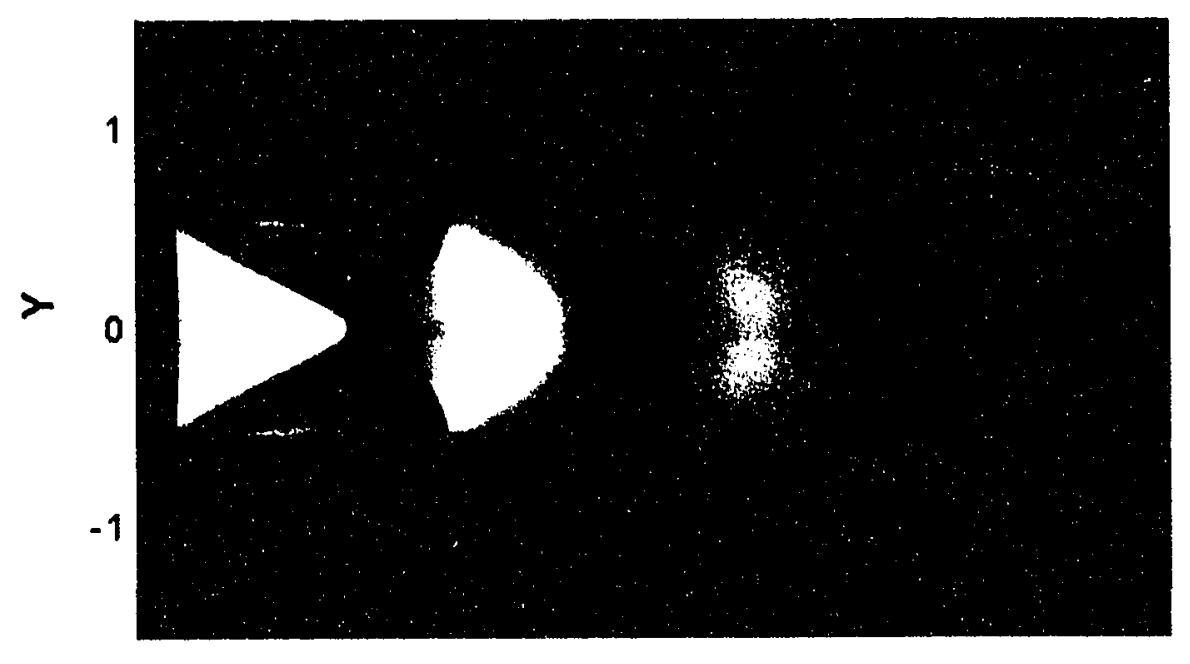

Figure 28: Numerical Schlieren for Mach $=1.43$

Figure 29 shows the time history of pressure at the control point $(x=10, y=$ 2), which is 6 diameters from the nozzle, since for this 2-D case, the nozzle extends to $x=4$. Figure 30 shows the sound pressure level at that point same point vs. frequency in Hertz. Pressure history is post processed by taking the FFT transform to obtain SPL. The highest amplitude occurs at $5390 \mathrm{~Hz}$, which corresponds to screech frequency. This result is in good agreement with experimental result of $5400 \mathrm{~Hz}$ in Panda (1999). These results were obtained without introducing any periodic forcing function. The inflow boundary conditions at the nozzle exit and the nozzle lip create instability vortices and the interaction of these with the shock cells generates screech waves. 


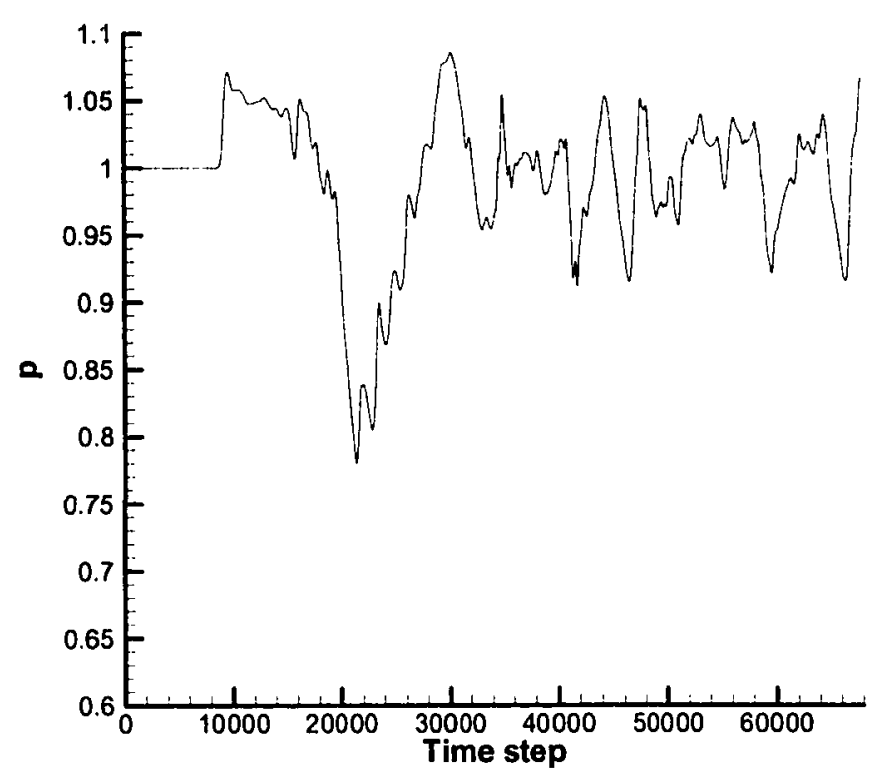

Figure 29: Pressure history at point $(10,2)$

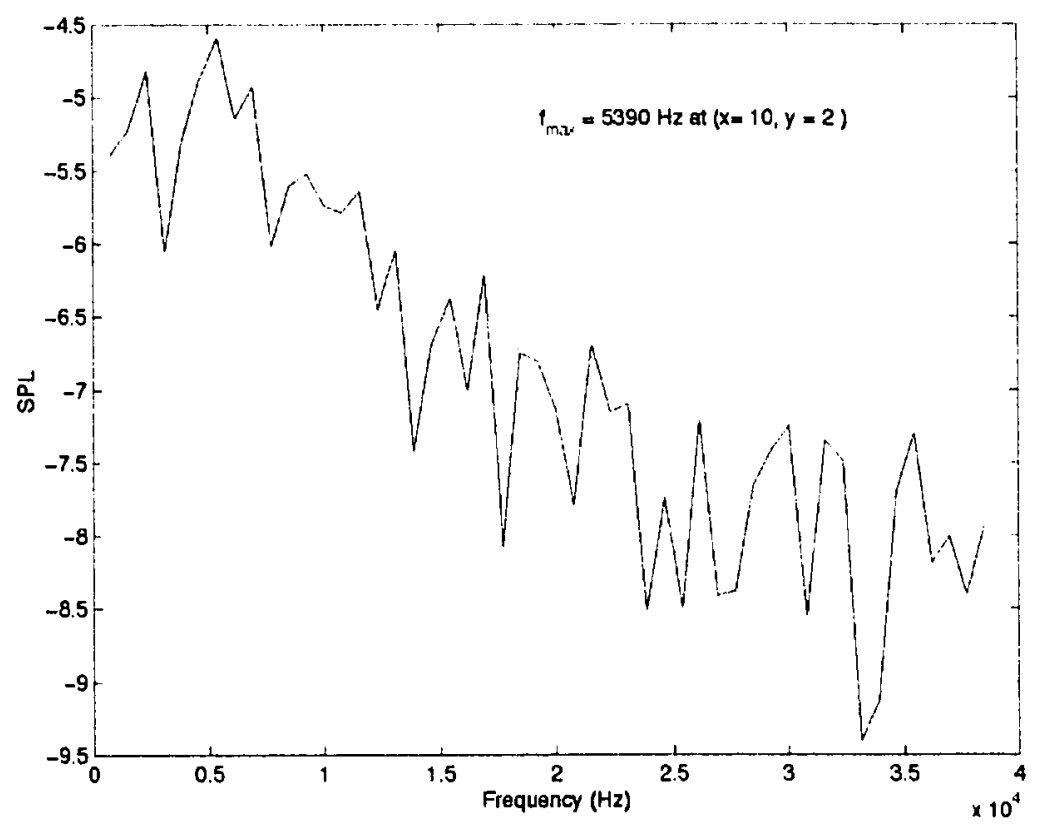

Figure 30: SPL plot for point $(10,2)$

Our three dimensional simulation results are seen in Fig. 31. Extension of the problem to the third dimension has produced similar results of shock cell and shear 
layer pattern. To understand what happens in the angular direction, we plot the density contours at $\theta=0$ and $\theta=90$ degrees in Figures 32 and 33. The difference is too small to observe in these plots. Fig. 34 gives a good comparison of the difference between two angles. The general flow characteristics are almost same for each $\theta$ plane except for small differences. Figures 35 and 36 show how the density varies with $\theta$ for selected fixed $\mathrm{x}$ and $\mathrm{r}$ values. The variation is almost like a sine wave in Fig. 35 and it is like a combination of two harmonics in Fig. 36. Unfortunately, we had to reduce the number of points and time steps to save computational resources, and we were not able to perform a 3-dimensional time series analysis. To see how this case compares to 2-dimensional and experimental results, we used experimental data from (Panda, Seasholtz, 1999), which is available on the internet (EPAPS). For two selected $x$ values, density vs. radial distance plots are generated in Figures 37 and 38 . The results suggest that both 2-D and 3-D computations are in good agreement with the experiment, except for a few data points close to centerline at $x=0.9$. Looking at these results, it may be concluded that even 2-D computations are sufficient to model helical mode screech. However, to make a better comparison, one must use same grid and time steps for both cases, except the azimuthal direction. It would take very long computational time for us to use 400 by 400 by 16 grid points, and the time step had to be also small to keep CFL number in range. As in the previous cases, we have shown that WENO scheme is very suitable and efficient for this kind of problem that involves moving shocks and vortices. 


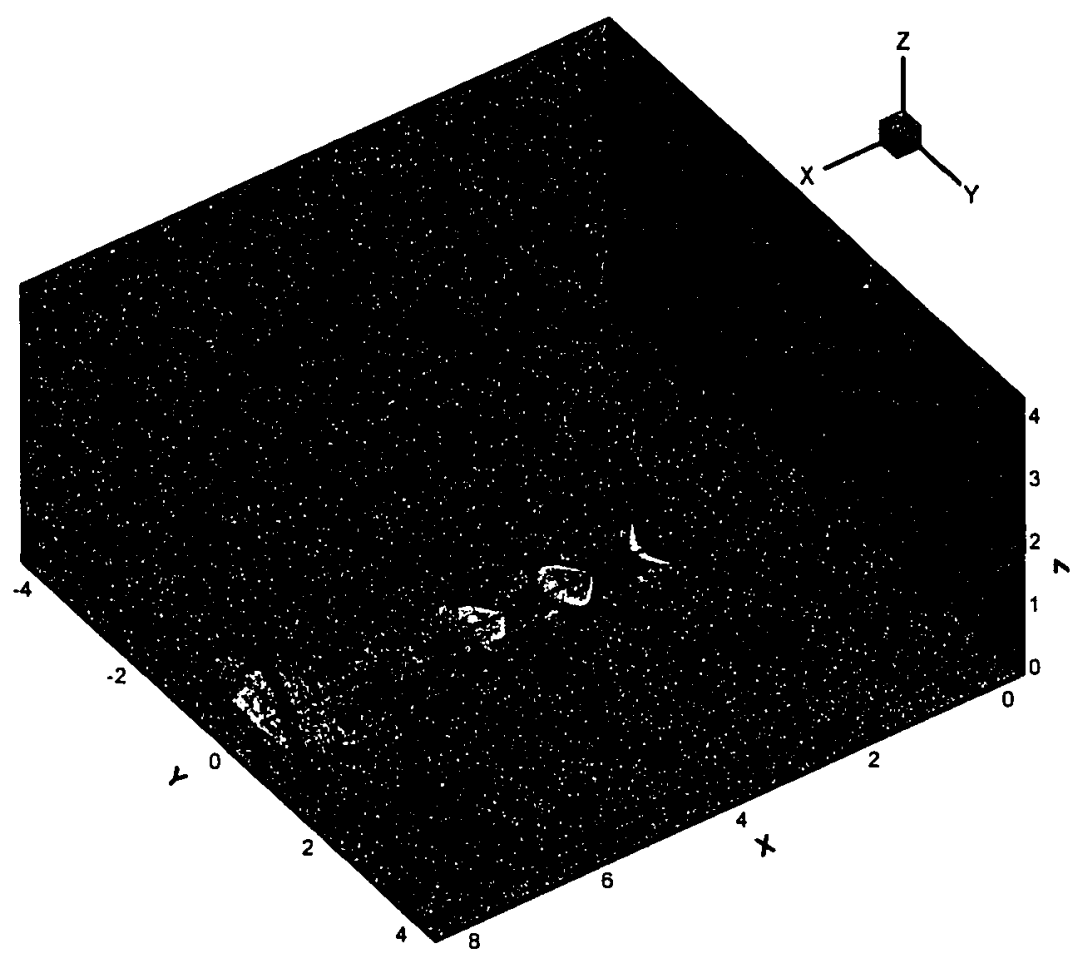

Figure 31: 3-D density contours for $\mathrm{Mach}=1.43$

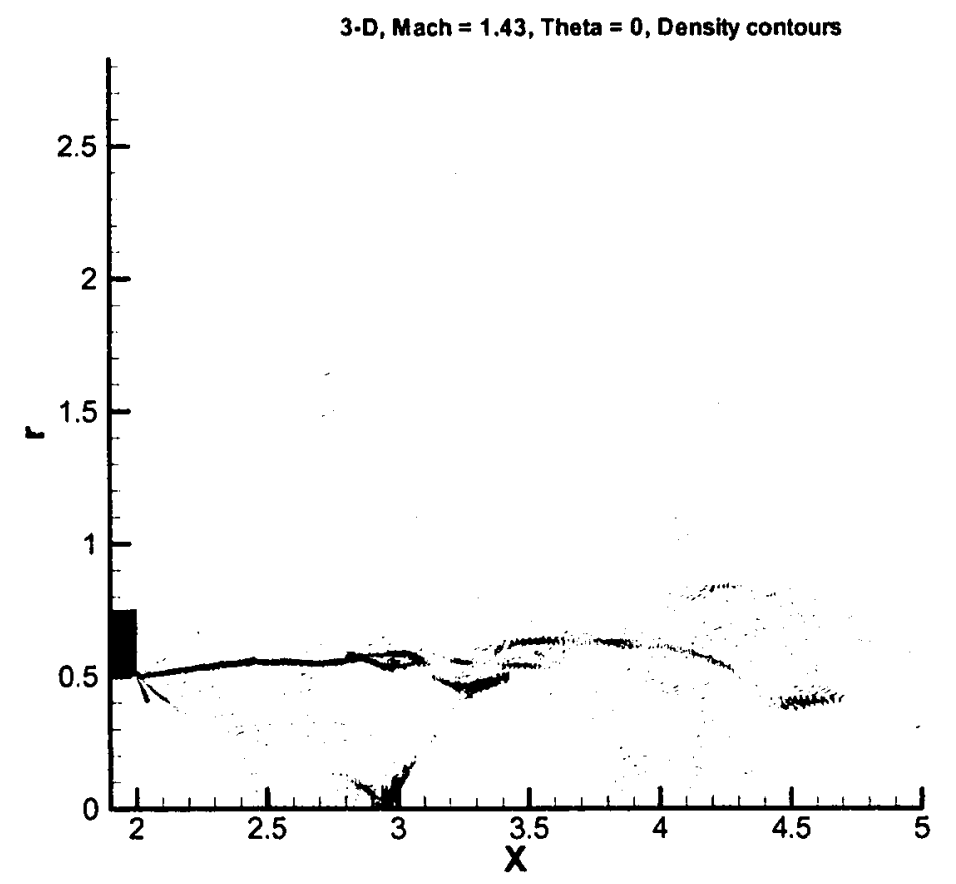

Figure 32: 3-D density contours for $M a c h=1.43, \theta=0$. 


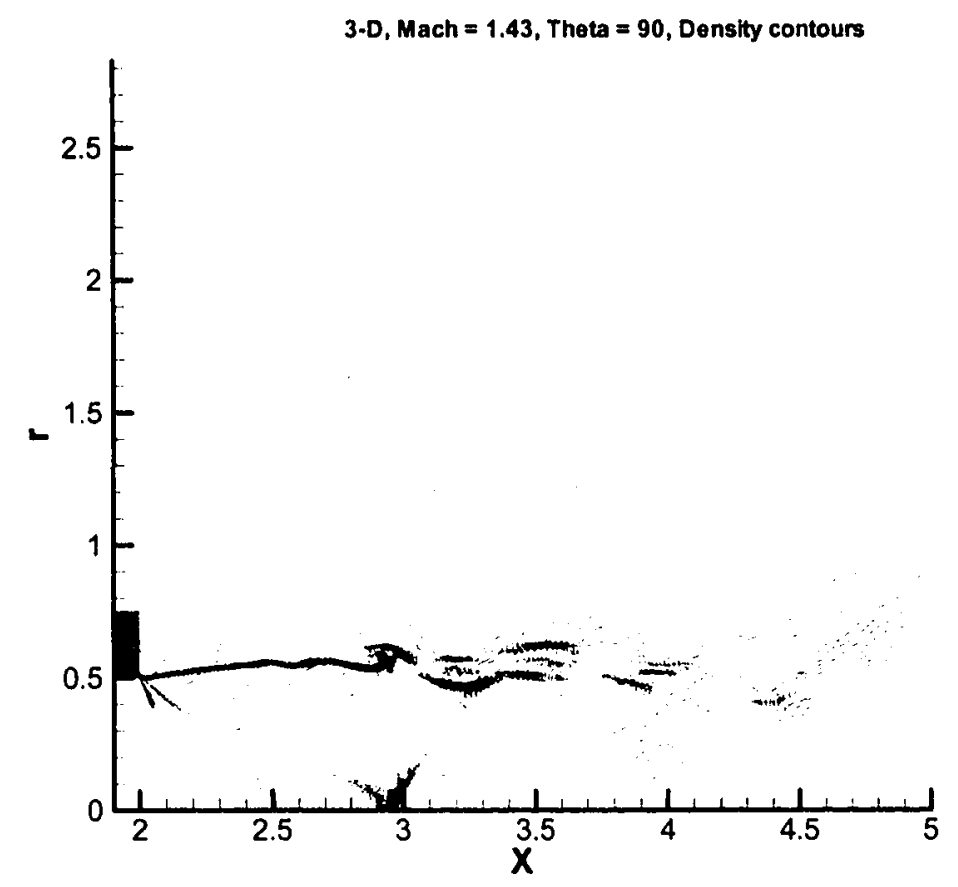

Figure 33: 3-D density contours for Mach $=1.43, \theta=90^{\circ}(\pi / 2)$.

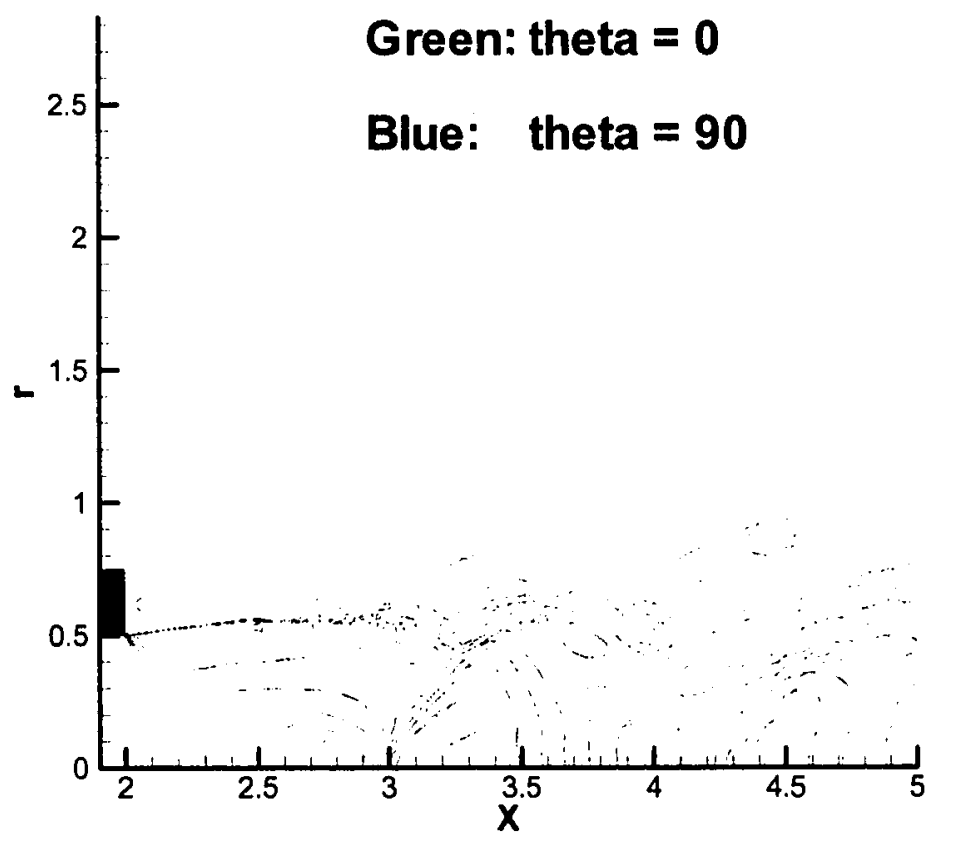

Figure 34: Comparison of Fig. 8 and Fig. 9, number of contours are reduced for clarity. 
$x=2.12, r=0.86$, density vs. theta

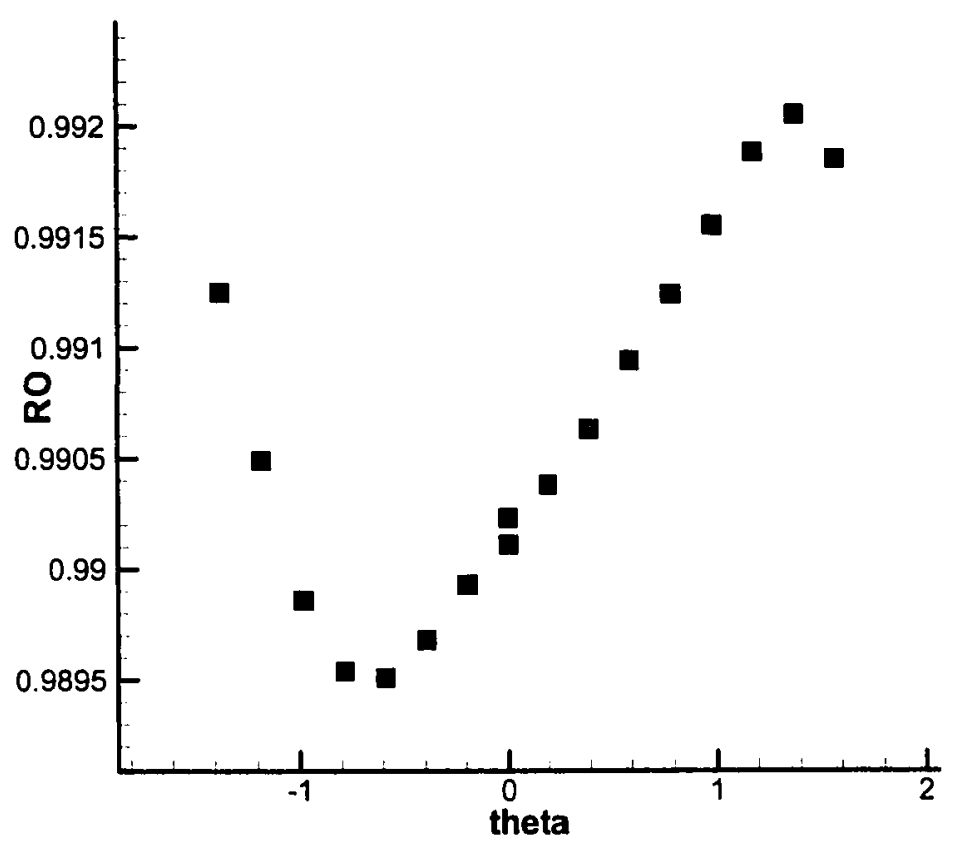

Figure 35: Variation of density in azimuthal direction at selected point $(2.12,0.86)$. 
$x=4.00, r=0.26$, density vs. theta

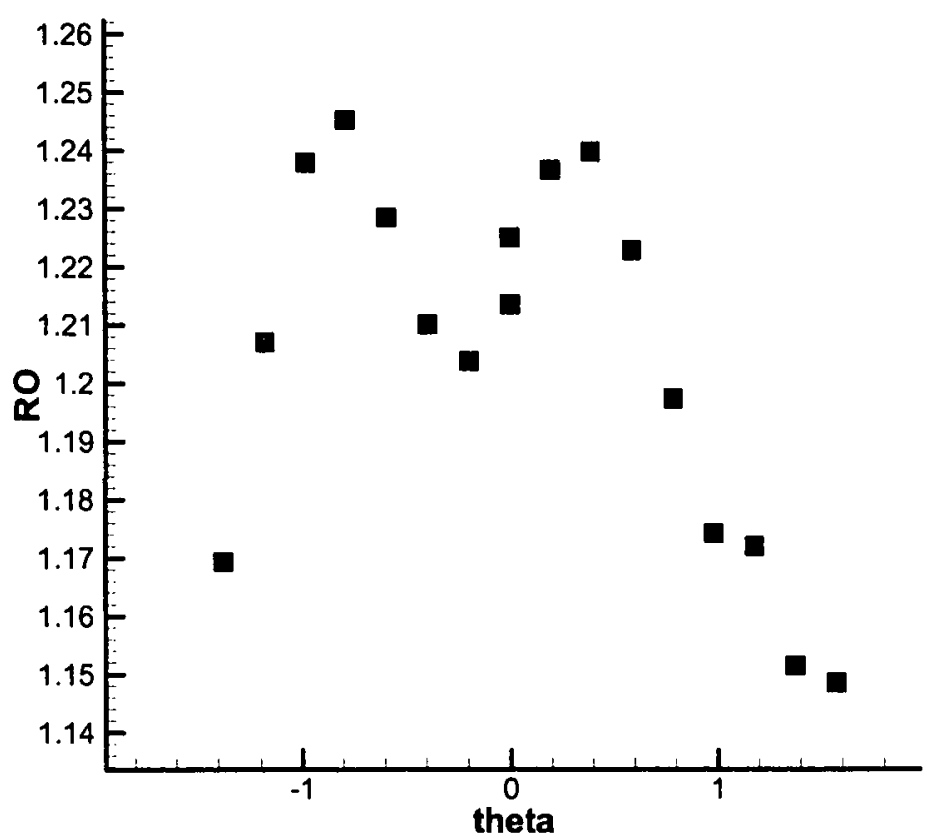

Figure 36: Variation of density in azimuthal direction at selected point $(4.00,0.26)$.

$$
x=0.6
$$

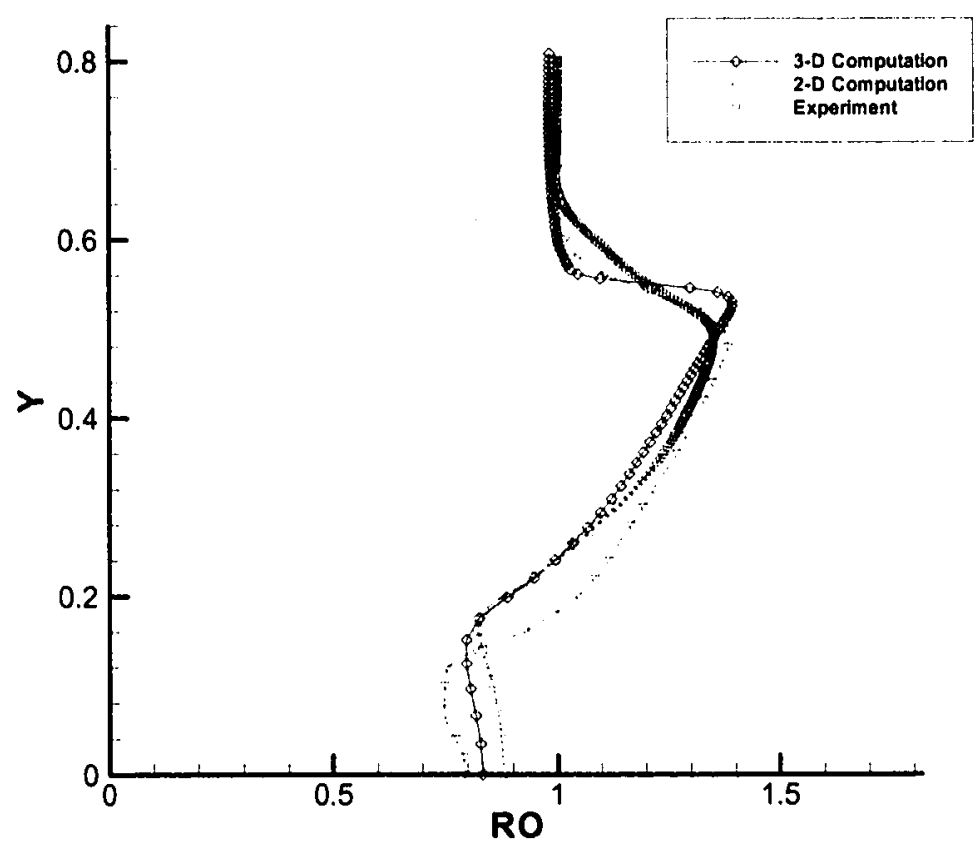

Figure 37: Comparison of 3-D and 2-D computations with experiment for Mach $=1.43$ at $x=0.6$ 


$$
x=0.90
$$

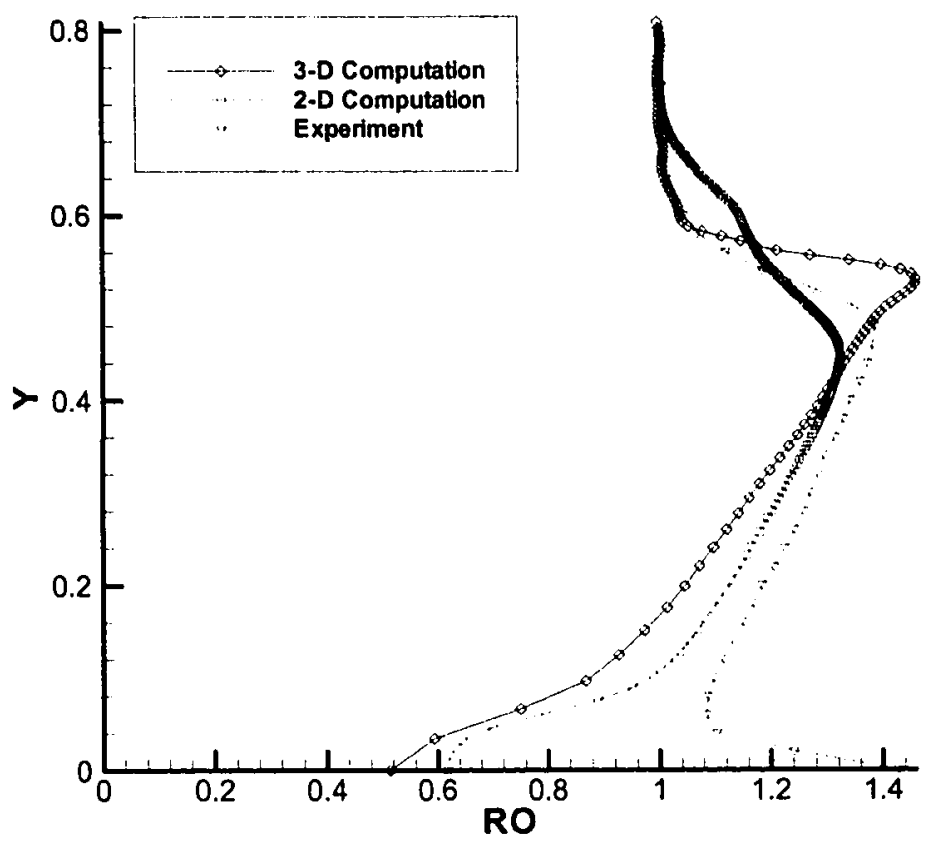

Figure 38: Comparison of 3-D and 2-D computations with experiment for Mach $=1.43$ at $x=0.90$ 


\section{CHAPTER 7 CONCLUSIONS AND RECOMMENDATIONS}

The problem of jet screech noise has been investigated numerically and experimentally since the 1950's. Recent experiments by Panda (1999) have provided valuable data for understanding screech noise generation mechanisms. This study has been done to verify and improve our understanding of screech generation.

Screech noise in underexpanded jets has been modeled using axisymmetric and three dimensional numerical models. Fifth order WENO scheme and third order TVD time discretization has been chosen for their fitness for this type of problem where strong moving shocks are present. Full Navier-Stokes equations have been solved in cylindrical coordinates. Smagorinsky's LES turbulence model has been used for axisymmetric computation. Non reflecting boundary conditions have been used at free boundaries.

The main emphasis is on the axisymmetric case, where the Mach number is 1.19 and three dimensional effects are not expected. This case has been modeled using 160,000 grid points, 400 in each direction. The helical mode case with Mach number 1.43 was solved using axisymmetric model first, and then using threedimensional model for comparison. The three dimensional case has been modeled using 200 by 150 in the axisymmetric plane and 16 points in the azimuthal direction, making 480,000 total points. The number of points had to be reduced to make up for the increase by 16 times from going to the third dimension. Good results were still obtained for that reduced grid. For 1.43 Mach case, the helical mode case produced very similar results to axisymmetric case. Another case with Mach number 1.80 was solved just for verification of different shock cell structure, the barrel shock, which was observed in the experiments; and it came out as expected. The structure of shock cells and their position has been captured precisely in all cases. The screech frequency has also been obtained very close to experimental results for both 1.19 and 1.43 Mach numbers, $8400 \mathrm{~Hz}$ and $5400 \mathrm{~Hz}$, respectively. Video animations created using the solutions at different time steps of both cases illustrate the interaction 
between shock cells and vortices, and propagating screech waves. The screech waves emerge from the second and third shock cells, like in the experiments. The screech wavelength can be roughly estimated as $1.5 \mathrm{D}$, which is close to other computational studies. The solution does not involve any artificial viscosity, tune up parameters, or any perturbation. The frequencies are obtained directly by numerical simulation.

The computational methods used in this study are good choices for this type of problems where moving sharp discontinuities occur. Shock capturing is automatic in all domain with this approach. Even coarser grids than used here give the shock cell structure accurately, but may fail to compute the frequency correctly. One disadvantage is that since the algorithm is explicit, the time step must be set small such that the CFL number is about 0.5 . The grid points at the nozzle lip are very fine, and that forces the time step to be very small to satisfy the CFL condition. Therefore, many time steps are required, making the computation time long.

The contribution of this study is that it is a method to calculate screech frequencies without artificial damping or other tune-up parameters. This is a direct numerical simulation, and the formulation is simpler than most of the other studies in this area. In addition, the visual data obtained by the animations has been useful to see shock generation locations.

For future work, running the 3-dimensional case with same number of planar points as the axisymmetric case may be a good comparison. Trying different mach numbers and nozzle geometries is possible. Increasing the domain size may be needed to eliminate reflections at higher Mach numbers. Turbulence model can be extended to 3-dimensions and higher order turbulence models like $k-\varepsilon, k-\omega$ can be tried. These require more computational resources than currently available. 


\section{REFERENCES}

Abgrall, R. (1994) "On Essentially Non-oscillatory Schemes on Unstructured

Meshes: Analysis and Implementation", Journal of Computational Physics, Vol. 114, pp. 45-58.

Adams, N., Shariff, K. (1996) "A High-Resolution Hybrid Compact-ENO Scheme for Shock-Turbulence Interaction Problems", Journal of Computational Physics, Vol. 127, pp 27-51.

Anderson, J. D. (1990) "Modern Compressible Flow: With Historical Perspective", Second Edition, McGraw-Hill.

Atkins, H. L., "High-Order ENO Methods for the Unsteady Compressible NavierStokes Equations", AIAA Paper 91-1557, 1991.

Bihari, B., Harten, A. (1989) "Application of Generalized Wavelets: An Adaptive Multiresolution Scheme”, Journal of Computational Physics, Vol. 85, pp. 275-321.

Cai, W., Shu, C. W. (1993) "Uniform High-order Spectral Methods for One- and Two-dimensional Euler Equations", Journal of Computational Physics, Vol. 104, pp. 427-443.

Cain, A. B. (1993) "Computation of Supersonic Jet Screech", ASME FED Vol. 147, pp. 123-129.

Casper J., Atkins, H. (1993) “A Finite Volume High-order ENO Scheme for Two Dimensional Hyperbolic Systems", Journal of Computational Physics, Vol. 106, pp. $62-76$. 
Casper, J., Carpenter, M. H. (1998) "Computational Considerations for the Simulation of Shock Induced Sound", SIAM Journal of Scientific Computing, Vol. 19, No. 3, pp. 813-828.

Christofi, S., "The Study of Building Blocks for ENO Schemes", Ph.D. Thesis, Division of Applied Mathematics, Brown University, September 1995.

Dolezal A., Wong, S. (1995) "Relativistic Hydrodynamics and Essentially Nonoscillatory Shock Capturing Schemes", Journal of Computational Physics, Vol. 120, pp. 266-277.

EPAPS Document No. E-PHFLE6-11-017912, http://www.aip.org/pubservs/epaps.html

Erlebacher, G., Hussaini, Y., Shu, C. W. (1997) "Interaction of a Shock with a Longitudinal Vortex", Journal of Fluid Mechanics, Vol. 337, pp. 129-153.

Fatemi, E., Jerome, J., Osher, S., (1991) "Solution of the Hydrodynamic Device Model Using High Order Non-Oscillatory Shock Capturing Algorithms", IEEE Transactions on Computer-Aided Design of Integrated Circuit and Systems, Vol. 10, pp. 232-244.

Gribben, B. J., Badcock, K. J., Richards, B. E. (2000) "Numerical Study of Shock Reflection in an Underexpanded Jet", AIAA Journal, Vol. 38, No. 2, pp. 275-283

Harten, A., Engquist, B., Osher, S., Chakravarthy, S. (1987) "Uniformly high order essentially non-oscillatory schemes, III", Journal of Computational Physics, Vol. 71, pp. 231-303.

Harten, A. (1989) "ENO Schemes with Subcell Resolution", Journal of Computational Physics, Vol. 83, pp. 148-184. 
Harabetian, E., Osher, S., Shu, C. W., (1996) “An Eularian Approach for Vortex Motion Using a Level Set Regularization Procedure", Journal of Computational Physics, Vol. 127, pp. 15-26.

Imamoglu, B., Balakumar, P., (2002) "Computation of Shock Induced Noise in Imperfectly Expanded Supersonic Jets", AIAA paper 2002-2527, presented at the 8th AIAA/CEAS Aeroacoustics Conference in Breckenridge, Colorado on 17 - 19 June 2002.

Imamoglu, B., Balakumar, P., (2003) "Three Dimensional Computation of Shock Induced Noise in Imperfectly Expanded Supersonic Jets", AIAA paper 2003-3249, presented at the 9th AIAA/CEAS Aeroacoustics Conference, Hilton Head, SC on 1214 May 2003.

Iske, A., Soner, T., (1996) "On the Structure of Function Spaces in Optimal Recovery of Point Functionals for ENO-schemes by Radial Basis Functions", Numerische Mathematik, Vol. 74, pp. 177-201.

Jiang, G., Shu, C. W. (1996) "Efficient Implementation of Weighted ENO Schemes", Journal of Computational Physics, Vol. 126, pp. 202-228.

Kim, J. W., Lee, D. J. (2000) "Generalized Characteristic Boundary Conditions for Computational Aeroacoustics", AIAA Journal, Vol. 38, No. 11, pp. 2040-2049.

Ladeinde, F., O’Brien E., Cai, X., Liu, W. (1995) "Advection by Polytropic Compressible Turbulence", Physics of Fluids, Vol. 7 (1995), pp. 2848-2857.

Liu, X. D., Osher, S., Chan, T. (1994) "Weighted Essentially Nonoscillatory Schemes”, Journal of Computational Physics, Vol. 115, pp. 200-212. 
Loh, C. Y., Himansu, A., Wang, X. Y., Jorgenson, P. C. E. (2001) "Computation of an Underexpanded 3-d Rectangular Jet by the CE/SE Method", AIAA paper 20010986.

Loh, C. Y., Hultgren, L. S., Jorgenson, P. C. E., "Near Field Screech Noise Computation for an Underexpanded Supersonic Jet by the CE/SE Method", Paper Draft.

Manning, T. A., Lele, S. K. (2000) "A Numerical Investigation of Sound Generation in Supersonic Jet Screech", AIAA paper 2000-2081

Norum, T. D., Seiner, J. M., "Location and Propagation of Shock Associated Noise from Supersonic Jets", AIAA paper 80-0983.

Panda, J. (1999) "An Experimental Investigation of Screech Noise Generation", Journal of Fluid Mechanics, vol. 378, pp 71-96.

Panda, J., Seasholtz, R. G., (1999) "Measurement of Shock Structure and ShockVortex Interaction in Underexpanded Jets Using Rayleigh Scattering", Physics of Fluids, vol. 11, Number 12, pp. 3761-3777.

Powell, A. (1953 a) "On the Noise Emanating from a Two-Dimensional Jet Above the Critical Pressure", Aeronautical Quarterly, Vol. IV, pp 103-122.

Powell, A. (1953 b) "On the Mechanism of Choked Jet Noise", Proceedings of the Physical Society (London), Vol. 66, pp 1039-1056.

Raman, G., Rice, E. J. (1994) "Instability Modes Excited by Natural Screech Tones in a Supersonic Rectangular Jet”, Physics of Fluids, 6, pp. 3999-4008. 
Shen, H., Tam, C.K.W. (1998) "Numerical Simulation of the Generation of Axisymmetric Mode Jet Screech Tones”, AIAA Journal, Vol. 36, No. 10, pp. 18011807.

Shu, C. W., Osher, S. (1989) "Efficient Implementation of Essentially Nonoscillatory Shock Capturing Schemes II", Journal of Computational Physics, Vol. 83, pp. 32-78.

Shu C. W. (1990) "Numerical Experiments on the Accuracy of ENO and Modified ENO Schemes", Journal of Scientific Computing, Vol. 5, pp. 127-149.

Shu, C. W., Zang, T. A., Erlebacher G., Whitaker D., Osher S. (1992), "High Order ENO Schemes Applied to Two- and Three-dimensional Compressible Flow", Applied Numerical Mathematics, Vol. 9, pp. 45-71.

Shu, C. W. (1997) "Essentially Non-Oscillatory and Weighted Essentially NonOscillatory Schemes for Hyperbolic Conservation Laws", ICASE Report No. 97-65.

Shu, W. E., Shu, C. W. (1994) "A Numerical Resolution Study of High Order Essentially Non-oscillatory Schemes Applied to Incompressible Flow", Journal of Computational Physics, Vol. 110, pp. 39-46.

Tam, C.K.W. (1994) "Jet Noise Generated by Large-Scale Coherent Motion", in "Aeroacoustics of Flight Vehicles, Theory and Practice--Volume 1: Noise Sources", Harvey H. Hubbard, Editor, pp. $311-390$.

Tam, C.K.W., Shen, H., Raman, G. (1997) "Screech Tones of Supersonic Jets from Bevelled Rectangular Nozzles", AIAA Journal, Vol. 35, No. 7, pp. 1119-1125.

Thompson, K. W. (1990) "Time Dependent Boundary Conditions for Hyperbolic Systems", Journal of Computational Physics, Vol. 89, No. 2, pp. 439-461. 
Walsteijn. F. (1994) "Robust Numerical Methods for 2D Turbulence", Journal of Computational Physics, Vol. 114, pp. 129-145.

Westley, R., Woolley, J. H. (1969) "The Near Field Sound Pressures of a Choked Jet During the Screech Cycle", in Aircraft Engine Noise and Sonic Boom, Paper 23, AGARD CP-42.

Yang, H. (1990) "An Artificial Compression Method for ENO Schemes, the Slope Modification Method", Journal of Computational Physics, Vol. 89, pp. 125-160. 


\section{VITA}

Bulent Imamoglu was born on January 30, 1973 in Istanbul, Turkey. He joined the Mechanical Engineering department of Bogazici University in 1991 and earned Bachelor of Science degree in 1995. After graduation, Mr. Imamoglu proceeded with the Masters program at the same department. He worked as a research and teaching assistant from 1996 to 1998. In 1998, he received his Master of Science in Mechanical Engineering. Then, in same year, Mr. Imamoglu joined the PhD. program at Old Dominion University, Aerospace Engineering Department. He works in the area of aeroacoustics. After graduation, he will return to Turkey to pursue his career. 